PNL--8532

DE93 008626

\title{
ENVIRONMENTAL SURVEILLANCE
}

MASTER SAMPLING SCHEDULE

L. E. Bisping

January 1993

Prepared for

the U.S. Department of Energy

under Contract DE-AC06-76RLO 1830

Pacific Northwest Laboratory

Richland, Washington 99352 


\section{SUMMARY}

Environmental surveillance of the Hanford Site and surrounding areas is conducted by the Pacific Northwest Laboratory $(P N L)^{(a)}$ for the U.S.

Department of Energy (DOE). Samples are routinely collected and analyzed to determine the quality of air, surface water, ground water, soil, sediment, wildlife, vegetation, foodstuffs, and farm products at Hanford Site and surrounding communities. This document contains the planned schedule for routine sample collection for the Surface Environmental Surveillance Project (SESP) and Drinking Water Project, and Ground-Water Surveillance Project.

The responsibility for monitoring the onsite drinking water falls outside the scope of the SESP. The Hanford Environmental Health Foundation is responsible for monitoring the nonradiological parameters defined in the National Drinking Water Standards while PNL conducts the radiological monitoring of the onsite drinking water. PNL has conducted the drinking water monitoring project concurrent with the SESP to promote efficiency and consistency, utilize the expertise developed aver the years, and reduce costs associated with management, procedure development, data management, quality control and reporting. As such, the 1993 drinking water sampling schedule is included in this document.

The ground-water sampling schedule is for 1993. This schedule identifies ground-water sampling events used by PNL for environmental surveillance of the Hanford Site. Some of these samples are collected and analyzed by other ground-water monitoring programs at Hanford (e.g. RCRA or CERCLA). They are indicated by a zero in the analysis column of the groundwater tables. Additional detail on the rationale for ground-water sampling is presented in Ground-Water Sample Collection and Analys is Plan for the Hanford Site Ground-Water Surveillance Project, R. W. Bryce, J. C. Evans, and K. C. 01 sen, 1991, PNL-7872, Pacific Northwest Laboratory, Richland, Washington.

This schedule is subject to modification during the year in response to changes in Site operations, program requirements, and the nature of the observed results.. Operational limitations such as weather, mechanical failures, sample availability, etc., may also require schedule modifications.

(a) Pacific Northwest Laboratory is operated by Battelle Memorial Institute for the U.S. Department of Energy under Contract DE-ACO6-76RLO 1830. 
Changes will be documented in the respective project files, but this schedule will not be reissued.

The purpose of these monitoring projects is to evaluate levels of radioactive and nonradioactive pollutants in the Hanford environs, as required in DOE Order 5400.1, "General Environmental Protection Program, " DOE Order 5400.5, "Radiation Protection of the Public and the Environment." The sampling methods will be the same as those described in the Environmental Monitoring Plan, United States Department of Energr, Richland Field Office, DOE/RL 91-50, U.S. Department of Energy, Richland, Washington.

Environmental and ground-water samples that are split with the Washington State Department of Health are indicated in the schedule as well as environmental samples that are split with the U.S. Food and Drug Administration.

Questions can be directed to Rodger Woodruff, Manager, Surface Environmental Surveillance Project, Roger Dirkes, Manager, Drinking Water Monitoring Project, and Robert Bryce, Manager, Ground-Water Environmental Surveillance Project. 


\section{CONTENTS}

SUMMARY

ABBREVIATIONS

viii

PART I. HANFORD SURFACE ENVIRONMENTAL SURVEILLANCE

1.0 AIR SURVEILlANCE ..................... 1

1.1 AIR - PARTICULATE FILTER . . . . . . . . . . . . 1

1.2 AIR - GASES AND VAPOR . . . . . . . . . . . . 3

2.0 SURFACE-WATER SURVEILLANCE $\ldots . . . . . . . . . . . .5$

2.1 WATER - COLUMBIA RIVER .............. 5

2.2 RIVERBANK SPRINGS ............... 6

2.3 ONSITE PONDS .................. . 6

2.4 OFFSITE WATER SYSTEMS . . . . . . . . . . . . . . 7

2.5 ONSITE DRINKING WATER ............... 7

3.0 FOODSTUFFS AND FARM PRODUCTS . . . . . . . . . . . 9

3.1 WHOLE MILK .................... 9

3.2 POULTRY AND EGGS . . . . . . . . . . . . . 9

3.3 BEEF . . . . . . . . . . . . . . . . 9

3.4 LEAFY VEGETABLES . . . . . . . . . . . . 10

3.5 VEGETABLES . . . . . . . . . . . . . 10

3.6 FRUIT .................... 11

3.7 WINE ..................... 11

3.8 WHEAT AND ALFALFA . . . . . . . . . . . . 12

4.0 WILDLIFE . . . . . . . . . . . . . . . . . . 14

4.1 AQUATIC BIOTA ................... 14

4.2 DUCKS .................... 15

4.3 GAME BIRDS . . . . . . . . . . . . . . 15

4.4 DEER ....................... 16 


\section{CONTENTS (contd)}

4.5 RABBITS ...................... 16

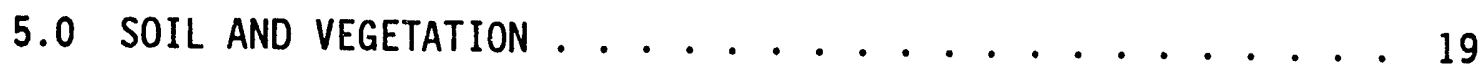

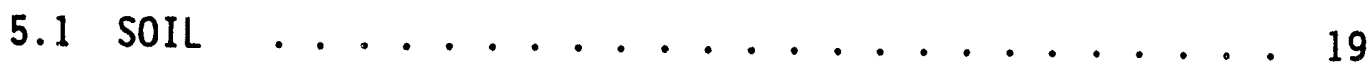

5.2 VEGETÁTION ................... 20

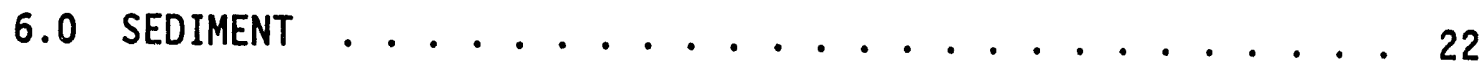

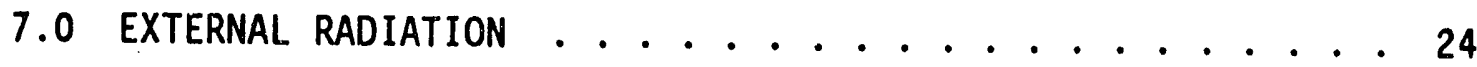

7.1 THERMOLUMINESCENT DOSIMETERS (TLDs) . . . . . . 24

7.1.1 Terrestrial Locations .......... 24

7.1.2 Columbia River Shoreline Locations . . . . 28

7.2 COLUMBIA RIVER SHORELINE RADIATION SURVEYS . . . . 29

7.3 ONSITE ROADWAY RADIATION SURVEYS .......... 31

7.4 ONSITE RAILWAY RADIATION SURVEYS ......... 32

7.5 ONSITE AERIAL RADIATION SURVEYS .......... 32

PART II. HANFORD GROUND-WATER SURVEILLANCE

1.0100 AREA WELLS ................. . . . . 37

$1.1100-B$ AREA WELLS ................ 37

$1.2100-D$ AREA WELLS ............. 37

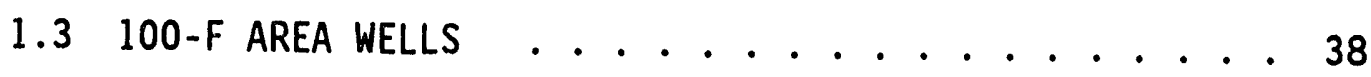

$1.4100-H$ AREA WELLS .................... 39

$1.5100-K$ AREA WELLS ............. . . 40

$1.6100-N$ AREA WELLS ............. 40

2.0200 AREA WELLS .................. 48

2.1200 -EAST AREA WELLS ............. . . . 48

2.2200 -WEST AREA WELLS ............ 51 
CONTENTS (contd)

3.01100 AND 300 AREA WELLS $\ldots \ldots . \ldots 56$

3.11100 AREA WELLS .............. 56

3.2300 AREA WELLS ............. 56

4.0400 AREA WELLS ....................... 59

5.0600 AREA WELLS ....................... 61 


\section{ABBREVIATIONS}

\section{FREQUENCY SYMBOLS USED}

$\begin{array}{ll}\text { A } & \text { annually } \\ \text { BA } & \text { biannually } \\ \text { BM } & \text { bimonthly (every } 2 \text { months) } \\ \text { BW } & \text { biweekly (every } 2 \text { weeks) } \\ \text { M } & \text { monthly } \\ \text { M Comp } & \text { monthly composite } \\ \text { Q } & \text { quarterly } \\ \text { Q Comp } & \text { quarterly composite } \\ \text { SA } & \text { semiannually } \\ \text { W } & \text { weekly }\end{array}$

\section{ANALYSIS SYMBOLS USED}

Generally, standard elements, chemical, and isotope designations are used to indicate the analyses performed. Other analysis designations used are:

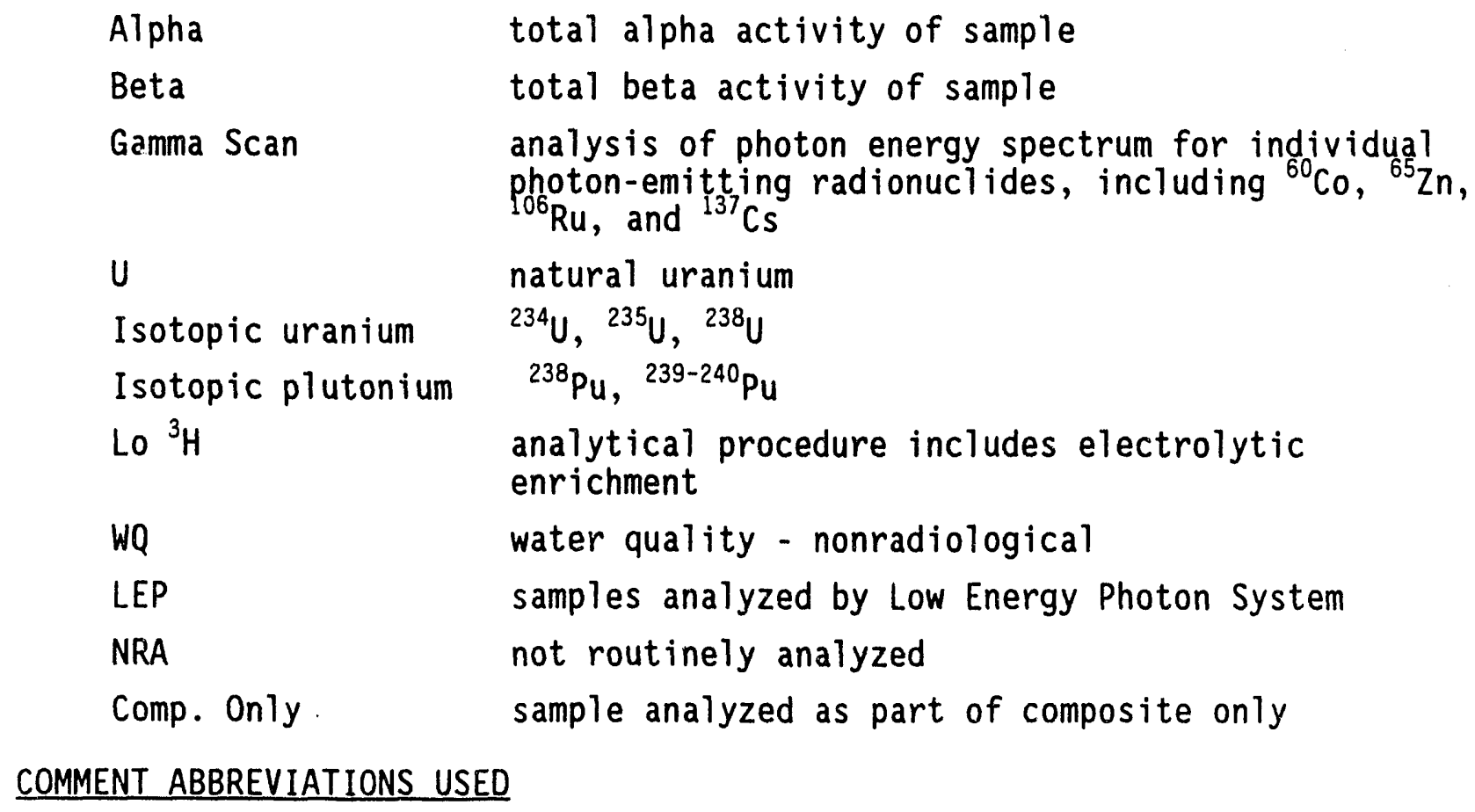

The abbreviations in the comment field for the Ground-Water Surveillance section are as designated: 


\section{ABBREVIATIONS (contd)}

Source

Plume

CAS

Water Supply

$\mathrm{DOH}$

Boundary

Background

3D-Char well being sampled to ilunitor a contaminant source area well used to track and define known contaminant plumes well sampled as part of a confined aquifer study water supply wells and wells near water supplies wells co-sampled with the Washington State Department of Health

wells at the Hanford Site boundary used to monitor the quality of water as it enters or leaves the Site

wells in uncontaminated areas of the Site used to identify concentrations of constituents in areas unaffected by Hanford operations

wells being screened to support an effort to characterize contaminant distribution in three dimensions

PORTABLE INSTRUMENT SYMBOLS USED

BICRON Micro rem meter

GM Geiger-Mueller counter

PIC Pressurized Ionization Chamber 


\section{PART I. HANFORD SURFACE ENVIRONMENTAL SURVEILLANCE}




\subsection{AIR SURVEILLANCE}

\subsection{AIR - PARTICULATE FILTER}

\section{ocation}

Location
Number(a)

Individual Samples

FreNumber quency

Analyses

Onsite

$100-k$

100-N (WPPSS)

100-D

$N$ of $200-E$

$N$ of 200-E \#2

$E$ of 200-E

200-ESE

$S$ of 200-E

$B$ Pond

B Pond No. 2

Army Loop Camp

200 Tel. Exchange

200-W SE

200-W SE No. 2

300 Water Intake

300 South Gate

300 Trench

300 NE

400-E

$400-W$

$400-5$

$400-\mathrm{N}$

Wye Barricade

Wye Barricade No. 2

$\begin{array}{lll}1 & 0058 & \\ 2 & 1529 & \\ 3 & 1074 & \\ 4 & 0051 & 8 \\ 4 & 0283 & \\ 5 & 0030 & \\ 6 & 0043 & 8 \\ 7 & 0031 & \\ 8 & 0038 & 8 \\ 8 & 0039 & 8\end{array}$

BW

Beta, Alpha Beta, Alpha 100 Areas Beta, Alpha

BW

NRA

BW

BW

BW

NRA

Beta, Alpha
Beta, Alpha

Beta, Alpha

200 East

BH

Beta, Alpha

Comp. Only

(200 East

8 Pond

0248

$10 \quad 0052$

BH

Beta, Alpha

200-W South 0614

Beta, Alpha and East

$11 \quad 0026$

$11 \quad 0027$

BW

Beta, Alpha

Comp. Only

200 West

1793

$13 \quad 6150$

BW

Beta

$14 \quad 1542$

$15 \quad 0022$

BH
BH

Beta, Alpha

300 Area

Beta, Alpha

Beta, Alpha

$300 \mathrm{NE}$

6308

6455

6456

6457

BW
BW
BW
BW

Beta, Alpha

Beta, Alpha

Beta, Alpha

Beta, Alpha

400 Area

0924

0036

BH

Beta, Alpha

Comp. Only

Wye

Barricade

Perimeter

Berg Ranch

Ringold Met Tower

Ringold Met Tower No.

22

22

$W$ End of Fir Rd

$W$ End of Fir Rd No. 2

$23 \quad 6391 \quad B W$

$230290 \quad B W$

$24 \quad 0255 \quad 8 W$

Dogwood Met Tower

Dogwood Met Tower No. 2
$24 \quad 0284 \quad B W$
NRA

Beta, Alpha

Comp. Only

Met Tower

Beta, Alpha W. End of

Comp. Only fir Road

0291

Beta, Alpha Dogwood Met. 0295 Comp. Only Tower

0286
Ringold
1753

Composited Sample

Sample Fre-

number quency

Analyses

1749

1738

0614

1739

1741

0298

6465

0601

Gamma Scan
A ${ }^{90} \mathrm{Sr}, \mathrm{Pu}(b), u^{(c)}$,
${ }_{241_{\mathrm{Am}}}$

Q Gamma Scan
A

Q Gamna Scan

A $90 \mathrm{Sr}, \mathrm{Pu}(\mathrm{b}), \mathrm{u}^{(c)}$,

Q Gamma Scan

A $\quad 241_{\mathrm{Am}}, \mathrm{Pu}(\mathrm{b}), U^{(c)}$, 


\subsection{AIR - PARTICULATE FILTER (contd)}

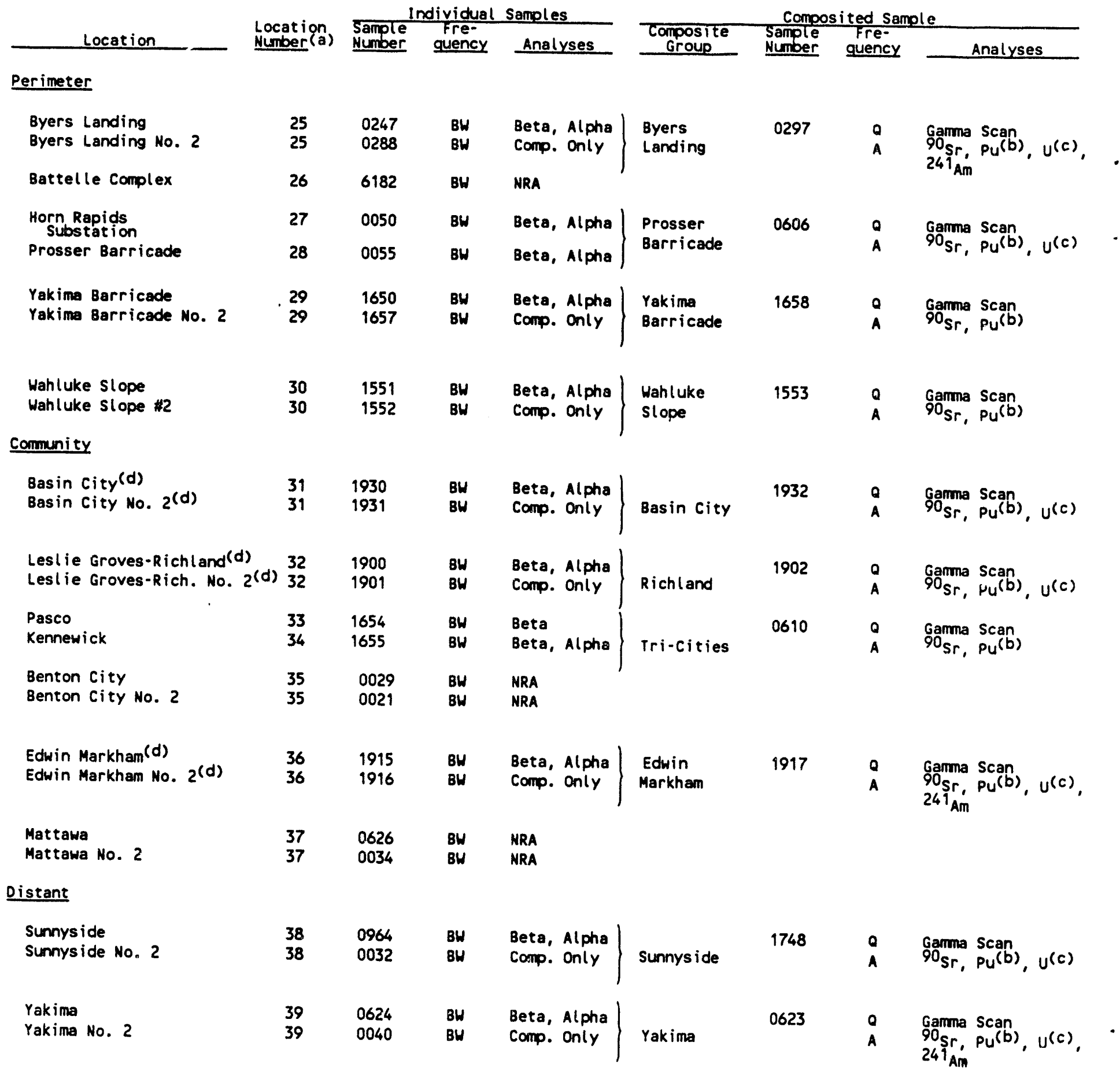

(a) Refer to Figure 1.1, Air Sampling locations.

(b) Isotopic plutonium.

(c) Isotopic uranium.

(d) Community-Operated Environmental Surveillance station. 


\subsection{AIR - GASES AND VAPOR}

\begin{tabular}{|c|c|c|c|c|c|c|c|}
\hline \multirow[b]{2}{*}{ Location } & \multirow[b]{2}{*}{$\begin{array}{l}\text { Locat ion } \\
\text { Number(a) }\end{array}$} & \multicolumn{2}{|c|}{131} & \multicolumn{2}{|c|}{$129_{1}$} & \multicolumn{2}{|c|}{$3_{H}$ (as HTO) } \\
\hline & & $\begin{array}{l}\text { Sample } \\
\text { Number }\end{array}$ & $\begin{array}{l}\text { Fre- } \\
\text { quency }\end{array}$ & $\begin{array}{l}\text { Sample } \\
\text { Number }\end{array}$ & $\begin{array}{l}\text { Fre- }(b) \\
\text { quency }\end{array}$ & $\begin{array}{l}\text { Sample } \\
\text { Number }\end{array}$ & $\begin{array}{l}\text { fre- } \\
\text { quenc }\end{array}$ \\
\hline Onsite & & & & & & & \\
\hline $\begin{array}{l}100-\mathrm{K} \\
200-\text { ESE } \\
200 \text { Tel. Exchange } \\
300 \text { South Gate } \\
300 \text { Trench } \\
300-\mathrm{NE} \\
400-\mathrm{E}\end{array}$ & $\begin{array}{r}1 \\
6 \\
10 \\
13 \\
14 \\
15 \\
16\end{array}$ & & & 0008 & Q Comp & $\begin{array}{l}0278 \\
6201 \\
0275 \\
0279 \\
0900 \\
6202 \\
6428\end{array}$ & $\begin{array}{l}M \\
M \\
M \\
M \\
M \\
M \\
M\end{array}$ \\
\hline
\end{tabular}

Perimeter

$\begin{array}{llllllll}\text { Ringold Met. Tower } & 22 & 0258 & \text { BW } & 0004 & \text { Q Comp } & 0906 & M \\ \text { Dogwood Rd. Met. Tower } & 24 & & & & & 0282 & M \\ \text { Byers Landing } & 25 & 0246 & \text { BW } & 0005 & \text { Q Comp } & 0273 & M \\ \text { Prosser Barricade } & 28 & & & & & 0908 & M \\ \text { Wahluke Slope } & 30 & & & & & 0266 & M\end{array}$

Community

$$
\begin{array}{ll}
\text { Basin City(C) } & 31 \\
\text { Leslie Groves-Richland(c) } & 32 \\
\text { Edwin Markham(c) } & 36
\end{array}
$$$$
1919 \text { BW }
$$

Distant

$\begin{array}{llllllll}\text { Sunnyside } & 38 & & & & & & \\ \text { Yakima } & 39 & 0630 & \text { BW } & 00010 & \text { Q Comp } & 0631 & M\end{array}$

(a) Refer to Figure 1.1, Air Sampling locations.

(b) Samples are collected monthly and compos i ted for quarterly analyses.

(c) Community-Operated Environmental Surveillance Station. 


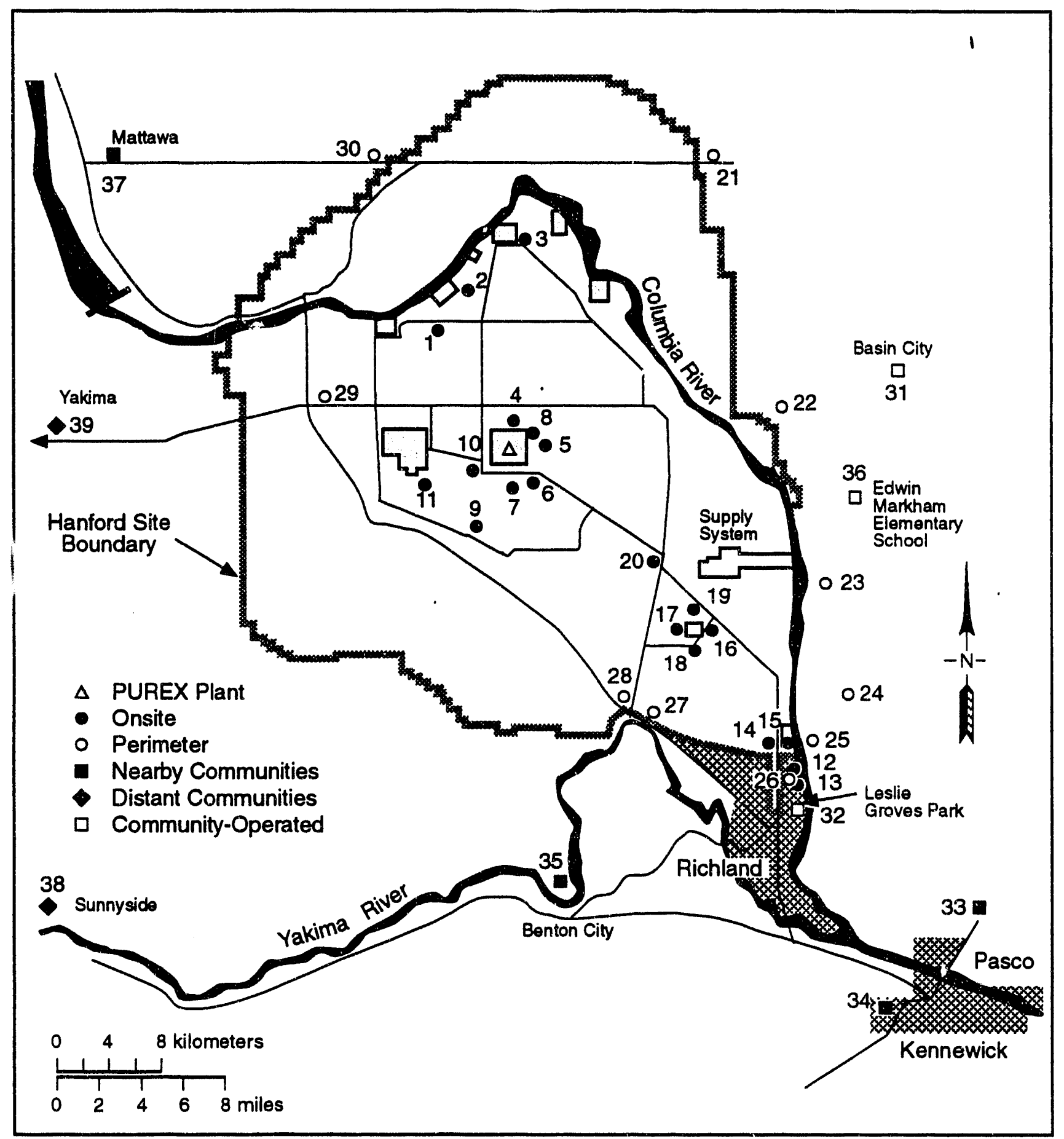

S9212076.4

Figure 1.1. Air Sampling Locations 


\subsection{SURFACE-WATER SURVEILLANCE}

\subsection{WATER - COLUMBIA RIVER}

\begin{tabular}{|c|c|c|c|c|}
\hline Location ${ }^{(a)}$ & Sample Type & Sample Number & Frequency & Analyses \\
\hline \multirow[t]{3}{*}{ Priest Rapids Dam } & Cumulative & 1265 & $M \operatorname{Comp}^{(b)}$ & $\begin{array}{l}\text { Alpha, Beta, }{ }^{\text {LO }}{ }^{3} \mathrm{H}, \text { Gamma } \\
\text { "can, } 90_{\mathrm{Sr}},{ }^{9} \mathrm{TC}, \mathrm{U}(\mathrm{c}), \mathrm{DOH}^{(d)}\end{array}$ \\
\hline & Particulate (filter) & 395 & $\begin{array}{l}M \operatorname{Comp}^{(e)} \\
0 \text { Comp }\end{array}$ & $\begin{array}{l}\text { Gamma Scan } \\
\mathrm{Pu}(f)\end{array}$ \\
\hline & Soluble (resin) & 6394 & $\begin{array}{l}\text { M Comp(e) } \\
0 \text { Comp }\end{array}$ & $\begin{array}{l}\text { Gamma Scan } \\
129_{1}, P_{u}(f)\end{array}$ \\
\hline \multirow[t]{2}{*}{ Vernita } & Grab & $\ldots(g)$ & 0 & $\begin{array}{l}\text { WQ-NASQAN, Temp, DO, Turbidity, } \\
\text { PH, Fecal Coliform, SS, DS, Cond, } \\
\text { Hardness as } \mathrm{CaCO}, \mathrm{P}, \mathrm{Cr} \text {, } \\
\mathrm{N}-\mathrm{K} \text { jeldahl, TOC, } \mathrm{NH}-\mathrm{N}\end{array}$ \\
\hline & Grab (Transect) & $0171-0174^{(h)}$ & $Q$ & $\begin{array}{l}\text { Lo }{ }^{3} \mathrm{H},{ }^{90} \mathrm{Sr}, U(\mathrm{c}) \text {, Volatile } \\
\text { Organics, ICP Metals, Anions }\end{array}$ \\
\hline $100-N$ & Grab (Transect) & $0140-0149^{(i)}$ & A & $\begin{array}{l}\text { Lo }{ }^{3} \mathrm{H},{ }^{90} \mathrm{Sr}, \mathrm{U}^{(\mathrm{c})} \text {, Volatile } \\
\text { Organics, ICP Metals, Anions }\end{array}$ \\
\hline $100-F$ & Grab (Transect) & $0191-0200^{(i)}$ & A & $\begin{array}{l}\text { Lo }{ }^{3} \mathrm{H},{ }^{90} \mathrm{Sr}, \mathrm{U}^{(\mathrm{C})} \text {, Volatile } \\
\text { Organics, ICP Metals, Anions }\end{array}$ \\
\hline Hanford Townsite & Grab (Transect) & $0410 \cdot 0419^{(i)}$ & A & $\begin{array}{l}\text { Lo }{ }^{3} \mathrm{H},{ }^{90} \mathrm{Sr}, \mathrm{U}^{(\mathrm{C})} \text {, Volatile } \\
\text { Organics, ICP Metals, Anions }\end{array}$ \\
\hline 300 Area & Grab (Transect) & $0420-0429^{(i)}$ & A & $\begin{array}{l}\text { Lo }{ }^{3} \mathrm{H},{ }^{90} \mathrm{Sr}, \mathrm{U}^{(\mathrm{c})} \text {, Volatile } \\
\text { Organics, ICP Metals, Anions }\end{array}$ \\
\hline \multirow[t]{6}{*}{ Richland Pumphouse } & Cumulative & 1001 & $\mathbf{H}$ & Alpha, Beta \\
\hline & Cumulative & 1000 & $M \operatorname{Comp}^{(b)}$ & 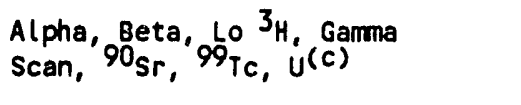 \\
\hline & Particulate (filter) & 6463 & $\begin{array}{l}\text { M Comp } \\
\text { Q Comp }\end{array}$ & $\begin{array}{l}\text { Gamma Scan } \\
\mathrm{Pu}^{(f)}\end{array}$ \\
\hline & Soluble (resin) & 6464 & $\begin{array}{l}\text { M Comp } \\
\text { Q Comp }\end{array}$ & $\begin{array}{l}\text { Gamma Scan } \\
129_{1}, P_{u}(f)\end{array}$ \\
\hline & Grab (Yraissect) & $0181 \cdot 0190^{(i)}$ & $a$ & $\begin{array}{l}\text { Lo } 3_{H},{ }^{90} \mathrm{Sr}, U(c) \text {, Volatile } \\
\text { Organics, ICP Metals, Anions }\end{array}$ \\
\hline & Grab & $\ldots(g)$ & $Q$ & $\begin{array}{l}\text { WQ-NASQAN, Temp, DO, Turbidity, } \\
\text { pH, Fecal Coliform, SS, DS, Cond, } \\
\text { Hardness as } \mathrm{CaCO}_{3}, \mathrm{P}, \mathrm{Cr}, \\
\mathrm{N}-\mathrm{K} \text { jeldahl, TOC, } \mathrm{Fe}, \mathrm{NH}_{3}-\mathrm{N}\end{array}$ \\
\hline
\end{tabular}

(a) Refer to Figure 2.1, Surface Water and Drinking Water Locations.

(b) Compos ite of weekly samples.

(c) Isotopic uranium.

(d) Dupl icate samples with the Washington State Department of Health (January and June only).

(e) Composite of bi weekly sample.

(f) Isotopic plutonium.

(g) Numerous water quality (wa) analyses are performed by the United States Geological Survey (USGS) in conjunction with the National stream Quality Accounting Network (NASOAN) Program.

(h) 4 samples collected along cross-section.

(i) 10 samples collected along cross-section. 


\subsection{RIVERBANK SPRINGS}

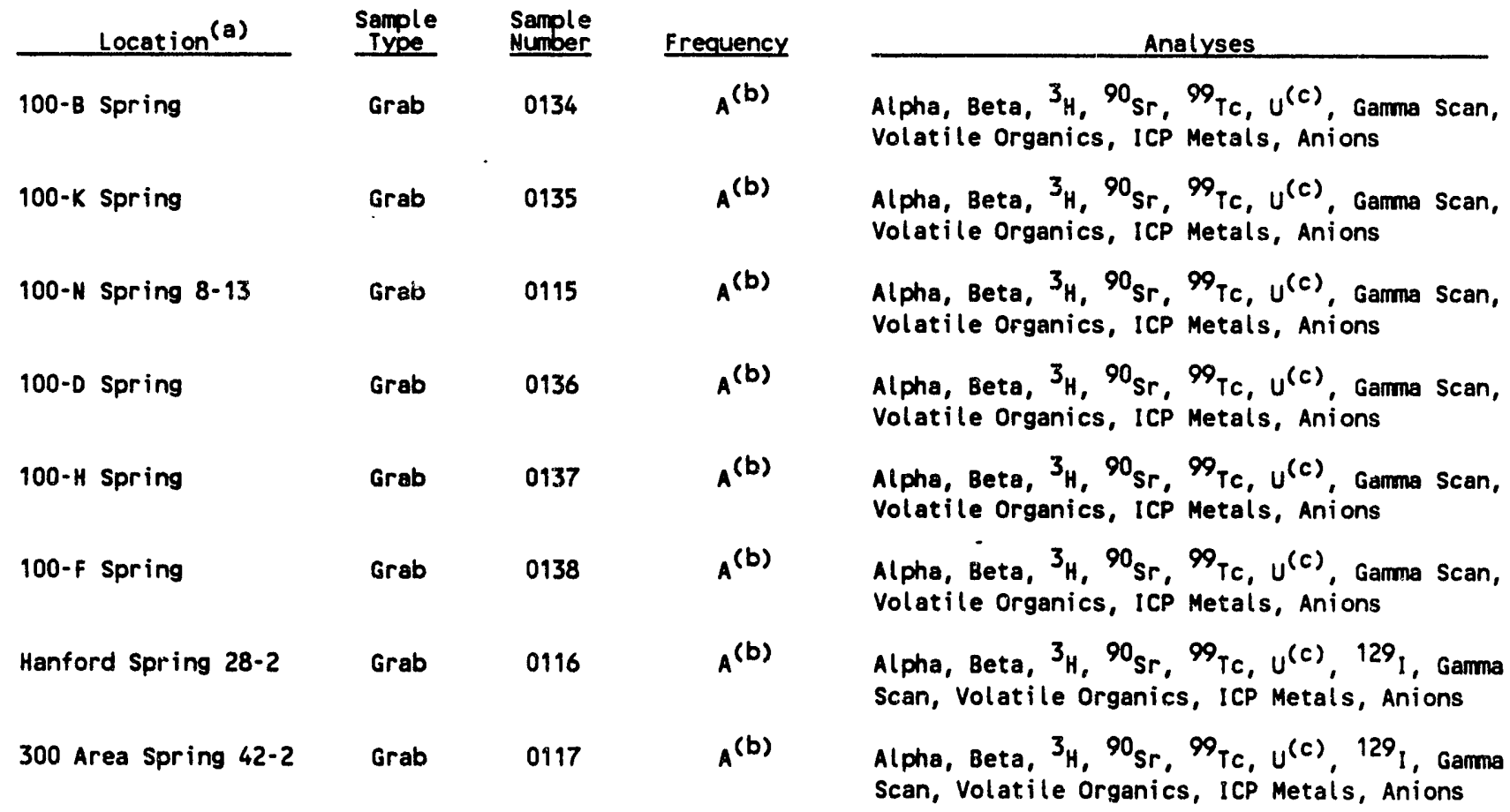

(a) Refer to Figure 2.1, Surface Water and Drinking Water Locations.

(b) Collected twice a year during low river flow season.

(c) Isotopic uranium.

\subsection{ONSITE PONDS}

\begin{tabular}{|c|c|c|c|c|}
\hline Location (a) & Sample rype & Sample Number & Frequency & Analyses \\
\hline West Lake & Grab & 6133 & $Q$ & $\begin{array}{l}\text { Alpha, Beta, }{ }^{3} \mathrm{H},{ }^{90} \mathrm{Sr},{ }^{99} \mathrm{Tc}, \mathrm{U}^{(b)} \text {, } \\
\text { Gamma Scan }\end{array}$ \\
\hline$B$ Pond & Grab & 0015 & $\mathbf{a}$ & Alpha, Beta, ${ }^{3} \mathrm{H},{ }^{90} \mathrm{Sr},{ }^{99} \mathrm{TC}$, Gamna Scan \\
\hline FFTF Pond & Grab & 6467 & a & Alpha, Beta, ${ }^{3} H$, Gamma Scan \\
\hline
\end{tabular}

(a) Refer to Figure 2.1, Surface Water and Drinking Water Locations.

(b) isotopic uranium. 


\subsection{OFFSITE WATER SYSTEMS}

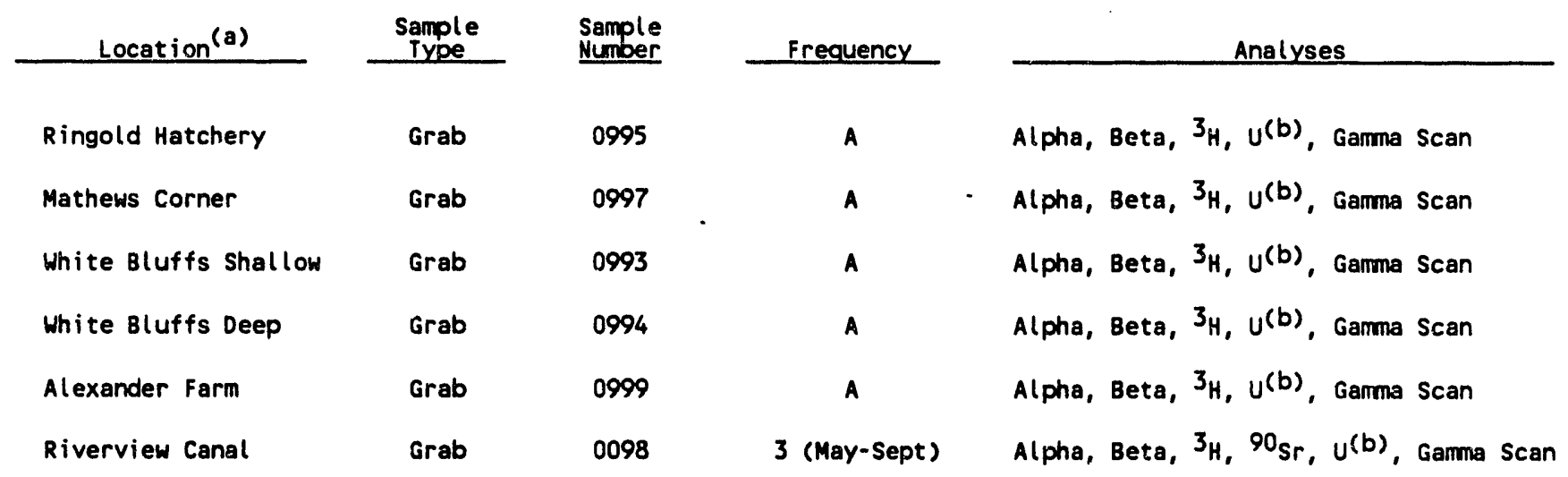

(a) Refer to Figure 2.i, Surface Water and Drinking Water Locations. (b) Isotopic uranium.

\subsection{ONSITE DRINKING WATER}

\begin{tabular}{|c|c|c|c|c|}
\hline Location (a) & $\begin{array}{c}\text { Sample } \\
\text { Type }\end{array}$ & $\begin{array}{l}\text { Sample } \\
\text { Number }\end{array}$ & Frequency & Analyies \\
\hline 100-B River & Grab & 6518 & 0 & Alpha, Beta, Lo ${ }^{3} \mathrm{H},{ }^{90} \mathrm{Sr}$, Gamma Scan \\
\hline 100-i River & Grab & 1219 & Q & Alpha, Beta, ${ }^{3} \mathrm{H}, 9^{90} \mathrm{Sr}$, Gamma Scan \\
\hline Yakima Barricade & Grab & 6543 & 0 & Alpha, Beta, ${ }^{3} \mathrm{H},{ }^{90} \mathrm{Sr}$, Gamma Scan \\
\hline Observatory & Grab & 6381 & 0 & Alpha, Beta, ${ }^{3} \mathrm{H},{ }^{90} \mathrm{Sr}$, Gamma Scan \\
\hline \multirow[t]{2}{*}{ FFTF } & Grab & 1220 & M & $\mathbf{3}_{\mathrm{H}}$ \\
\hline & & & Q & $\begin{array}{l}\text { Alpha, Beta, }{ }^{90} \mathrm{Sr} \text {, Gamma Scan } \\
129 \text { I }\end{array}$ \\
\hline 300 Water Intake & Cumulative & 6243 & Q Comp(b) & $\begin{array}{l}\text { Alpha, Beta, Lo }{ }^{3} \mathrm{H},{ }^{90} \mathrm{Sr},{ }^{99} \mathrm{TC} \text {, } \\
\mathrm{u}(\mathrm{c}) \text {, Gamma Scan }\end{array}$ \\
\hline Firing Range & Grab & 0099 & 0 & Alphs, Beta, ${ }^{3} \mathrm{H},{ }^{90} \mathrm{Sr}$, Gamma Scan \\
\hline ALE Headquarters & Grab & 6299 & Q & Alpha, Beta, ${ }^{3} \mathrm{H},{ }^{90} \mathrm{Sr}$, Gamma Scan \\
\hline
\end{tabular}

(a) Refer to Figure 2.1, Surface Water and Drinking Water Locations. (b) Composite of monthly cumulat ive samples.

(c) Isotopic uranium. 


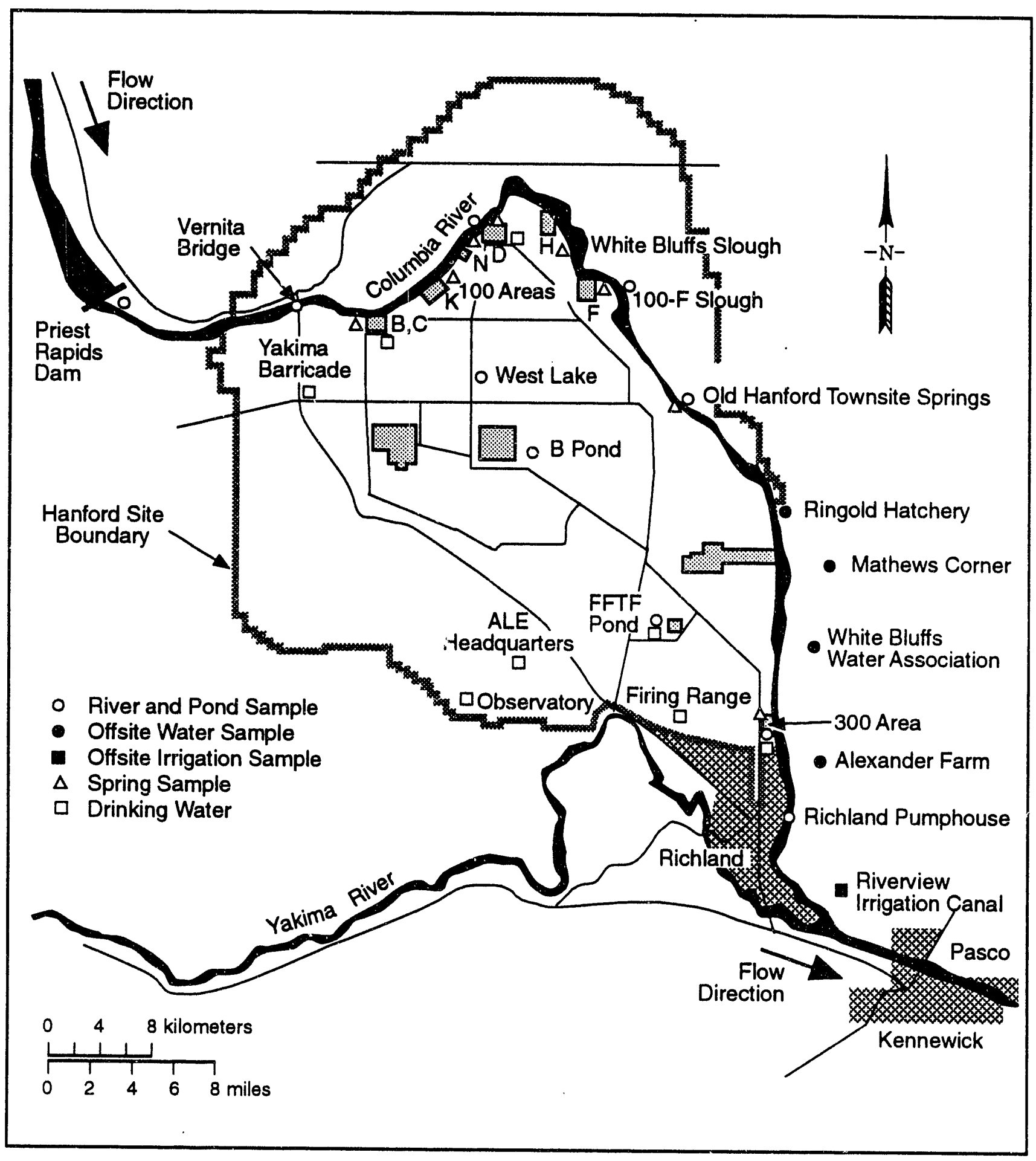

S9212076.5

Figure 2.1. Surface Water and Drinking Water Locations 


\subsection{FOODSTUFFS AND FARM PRODUCTS}

\subsection{WHOLE MILK}

Location (a)

East Wahluke Area Composite(b)

Sagemoor Area Composite(b)

Sunnyside Area

\author{
Sample Number
}

0305

0306

6355
Frequency

a

SA

$M$
SA

a

SA
Analyses

${ }_{126_{1}^{3}}{ }^{90} \mathrm{Sr}$, Gamma Scan

3H, Gamma Scan

$90 \mathrm{Sr}$
129

${ }_{126,}^{3_{\mathrm{H}}}{ }^{90} \mathrm{Sr}$, Gamma Scan

(a) Refer to Figure 3.1, Food and Farm Product Sampling Locations.

(b) Sample composited from three sources in each area.

\subsection{POULTRY AND EGGS}

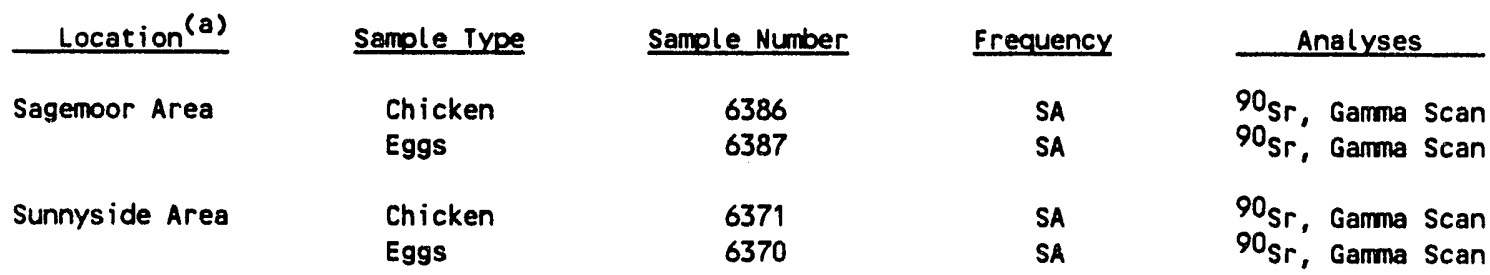

(a) Refer to Figure 3.1, Food and Farm Product Sampling Locations.

\subsection{BEEF}

Location (a)

Sagemoor Area

Riverview Area

Sunnyside Area
Sample Number

6418

1292

0322
Frequency

A

A

A
Analyses

${ }^{90}$ Sr, Gamma Scan

90 Sr, Gamma Scan, DOH ${ }^{(b)}$

${ }^{90} \mathrm{Sr}$, Gamma Scan, $\mathrm{DOH}(b)$

(a) Refer to Figure 3.1, Food and Farm Product Sampling Locations.

(b) Duplicate samples with the Hashington State Department of Heal th. 


\subsection{LEAFY VEGETABLES}

\begin{tabular}{|c|c|c|c|}
\hline Location (a) & Sample Number & Erequency ${ }^{(b)}$ & Analyses \\
\hline East Wahluke Area & 0308 & BA & ${ }^{90}$ Sr, Gamma Scan \\
\hline Sagemoor Area & 6419 & A & ${ }^{90} \mathrm{Sr}$, Gamma Scan, $129_{1}$-LEP \\
\hline Riverview Area & 1609 & A & ${ }^{90} \mathrm{Sr},{ }^{99} \mathrm{TC}$, Garma Scan, FDA $(\mathrm{c})$ \\
\hline Sunnyside Area & 6372 & A & ${ }^{90} \mathrm{Sr}$, Gamma Scan, FDA $(\mathrm{C})$ \\
\hline
\end{tabular}

(a) Refer to Figure 3.1, Food and Farm Product Sampling Locations.

(b) Three samples collected within each area. Biannual samples will next be collected in 1994.

(c) Duplicate samples sent to U.S. Food and Drug Adninistration.

\subsection{VEGETABLES}

\begin{tabular}{|c|c|c|c|c|}
\hline Location (a) & Sample Type & Sample Number & Frequency ${ }^{(b)}$ & Analyses \\
\hline Riverview Area & $\begin{array}{l}\text { Potatoes } \\
\text { Tomatoes } \\
\text { Carrots }\end{array}$ & $\begin{array}{l}0009 \\
0011 \\
0013\end{array}$ & $\begin{array}{l}A \\
A \\
A\end{array}$ & 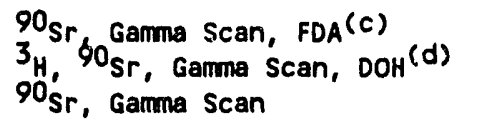 \\
\hline Horn Rapids & Potatoes & 0536 & A & $\begin{array}{l}{ }^{90} \mathrm{Sr},{ }^{99} \mathrm{TC}, \mathrm{Pu}^{(e)}{ }^{\left({ }^{(2 f}\right)} \mathrm{U}^{(f)} \\
\text { Gama Scan, } \mathrm{DOH}^{(d)}\end{array}$ \\
\hline Sagemoor Area & Potatoes & 0538 & A & ${ }^{90} \mathrm{Sr}, \mathrm{Pu}(e)$, Garma Scan, $F D A(c)$ \\
\hline Sunnyside Area & Potatoes & 0537 & A & $\begin{array}{l}{ }^{90} \mathrm{Sr}, \mathrm{Pu}(e), \mathrm{U}^{(f)}{ }^{99} \mathrm{TC}, \\
\text { Gamma Scan, FOA }(c)\end{array}$ \\
\hline Harrah/Wapato Area & $\begin{array}{l}\text { Potatoes } \\
\text { Tomatoes }\end{array}$ & $\begin{array}{l}0534 \\
0535\end{array}$ & $\hat{A}$ & $\begin{array}{l}90 \mathrm{Sr} \\
3_{\mathrm{H}},{ }^{\mathrm{GO}} \mathrm{Sr} \text {, Gamma Scan, } \mathrm{DOH}(\mathrm{g}) \\
\mathrm{DCH}, \mathrm{DOH}\end{array}$ \\
\hline East Wahluke Area & Potatoes & 0539 & BA & ${ }^{90} \mathrm{Sr}$, Gamma Scan \\
\hline
\end{tabular}

(a) Refer to Figure 3.1 , Foud and Farm Product Sampling Locations.

(b) Three samples of each type collected within each area. Biannual samples will next be collected in 1994.

(c) Duplicate samples sent to U.S. Food and Drug Administration.

(d) Dupl icate samples with the Washington State Department of Health.

(e) Isotopic plutonium.

(f) Isotopic uranium.

(g) Samples provided to PNL by Washington State Department of Health. 


\subsection{FRUIT}

\begin{tabular}{|c|c|c|c|c|c|}
\hline Location (a) & Sample Type & $\begin{array}{l}\text { Sample } \\
\text { Number }\end{array}$ & $\begin{array}{c}\text { Fre- } \\
\text { quency }\end{array}$ & $\begin{array}{l}\text { Collection } \\
\text { Period } \\
\end{array}$ & Analyses \\
\hline Sagemoor Area & $\begin{array}{l}\text { Apples } \\
\text { Cherries } \\
\text { Concord Grapes (e) }\end{array}$ & $\begin{array}{l}0565 \\
0543 \\
6417\end{array}$ & $\begin{array}{l}A \\
A \\
A\end{array}$ & $\begin{array}{l}\text { September } \\
\text { June } \\
\text { September }\end{array}$ & $\begin{array}{l}3_{H}, 90 \mathrm{Sr}, \text { Pu(c) , Gamma Scan, FDA }(d) \\
3_{H}, 90 \mathrm{Sr} \text {, Gamma Scan } \\
3_{H}, 90_{\mathrm{Sr}} \text {, Gamma Scan, }{ }^{129} 9_{I-L E P}\end{array}$ \\
\hline Cold Creek Area & $\begin{array}{l}\text { Apples } \\
\text { Concord Grapes (e) }\end{array}$ & $\begin{array}{l}0304 \\
0303\end{array}$ & $\begin{array}{l}\text { BA } \\
\text { BA }\end{array}$ & $\begin{array}{l}\text { September } \\
\text { September }\end{array}$ & $\begin{array}{l}3_{\mathrm{H}},{ }^{90} \mathrm{Sr} \text {, Gamma Scan } \\
3_{\mathrm{H}}{ }^{90} \mathrm{Sr} \text {, Garma Scan }\end{array}$ \\
\hline Sunnyside Area & $\begin{array}{l}\text { Apples } \\
\text { Cherries } \\
\text { Concord Grapes(e) }\end{array}$ & $\begin{array}{l}6440 \\
6453 \\
6454\end{array}$ & $\begin{array}{l}A \\
A \\
A\end{array}$ & $\begin{array}{l}\text { September } \\
\text { June } \\
\text { September }\end{array}$ & $\begin{array}{l}{ }^{3} \mathrm{H},{ }^{90} \mathrm{Sr} \text {, Pu(c), Gamma Scan } \\
3_{\mathrm{H},}{ }^{90} \mathrm{Sr} \text {, Gamma Scan } \\
3_{\mathrm{H},}{ }^{90} \mathrm{Sr} \text {, Gamma Scan, }{ }^{129} \text { I-LEP }\end{array}$ \\
\hline Riverview Area & $\begin{array}{l}\text { Apples } \\
\text { Concord Grapes(e) } \\
\text { Melons }\end{array}$ & $\begin{array}{l}0541 \\
0542 \\
0012\end{array}$ & $\begin{array}{l}A \\
A\end{array}$ & $\begin{array}{l}\text { September } \\
\text { September } \\
\text { August }\end{array}$ & $\begin{array}{l}3_{H}, 90 \mathrm{Sr} \text {, Gamma Scan, FDA }(d) \\
3_{H}, 90 \mathrm{Sr} \text {, Gamma Scan } \\
3_{\mathrm{H}},{ }^{90} \mathrm{Sr} \text {, Gamma Scan, DOH }\end{array}$ \\
\hline Ringold Area & $\begin{array}{l}\text { Apples } \\
\text { Cherries }\end{array}$ & $\begin{array}{l}0550 \\
0551\end{array}$ & $\hat{A}$ & $\begin{array}{l}\text { September } \\
\text { June }\end{array}$ & $\begin{array}{l}3_{H},{ }^{90} \mathrm{Sr}, \text { PU(C), Gamma Scan } \\
3_{\mathrm{H}}{ }^{90} \mathrm{Sr} \text {, Gamma Scan }\end{array}$ \\
\hline Harrah/Wapato Area & Melons & 0533 & A & August & ${ }^{3} \mathrm{H},{ }^{90} \mathrm{Sr}$, Gamma Scan, $\mathrm{DOH}^{(g)}$ \\
\hline Mattawa Area & $\begin{array}{l}\text { Apples } \\
\text { Concord Grapes (e) }\end{array}$ & $\begin{array}{l}0563 \\
0564\end{array}$ & $\begin{array}{l}\text { BA } \\
\text { BA }\end{array}$ & $\begin{array}{l}\text { September } \\
\text { September }\end{array}$ & $\begin{array}{l}{ }^{3} \mathrm{H},{ }^{90} \mathrm{Sr} \text {, Gamma Scan } \\
3_{\mathrm{H}}{ }^{90}{ }_{\mathrm{Sr}} \text {, Gamma Scan }\end{array}$ \\
\hline East Wahluke Area & Apples & 0540 & BA & September & ${ }^{3} H,{ }^{90} S r$, Gamma Scan, FDA $(d)$ \\
\hline
\end{tabular}

(a) Refer to Figure 3.1, Food and Farm Product Sampl ing Locations.

(b) Three samples of each type collected within each area. Biannual samples will next be collected in 1994.

(c) Isotopic plutonium

(d) Duplicate samples sent to the U.S. Fooc' and Drug Administration.

(e) Concord grapes preferred; table grapes acceptable if concord grapes are unavailable.

(g) Samples provided to PNL by Washington sjtate Department of Heal th.

\subsection{WINE}

\begin{tabular}{|c|c|c|c|c|c|}
\hline Location (a) & $\begin{array}{l}\text { Sample } \\
\text { Iype } \\
\end{array}$ & $\begin{array}{l}\text { Sample } \\
\text { Number }\end{array}$ & Erequency (b) & $\begin{array}{c}\text { Collection } \\
\text { Period } \\
\end{array}$ & Analyses \\
\hline Columbia Basin & $\begin{array}{l}\text { White } \\
\text { Red }\end{array}$ & $\begin{array}{l}0544 \\
0546\end{array}$ & $\begin{array}{l}A \\
A\end{array}$ & $\begin{array}{l}\text { December } \\
\text { December }\end{array}$ & $\begin{array}{l}3_{H} \text {, Gamma Scan, DOH(c) } \\
3_{H} \text {, Gamma Scan, DOH(c) }\end{array}$ \\
\hline Yakima Valley & $\begin{array}{l}\text { White } \\
\text { Red }\end{array}$ & $\begin{array}{l}0545 \\
0547\end{array}$ & $\begin{array}{l}A \\
A\end{array}$ & $\begin{array}{l}\text { December } \\
\text { December }\end{array}$ & $\begin{array}{l}3_{H} \text {, Garma Scan, } \mathrm{DOH}^{(c)} \\
3_{\mathrm{H}, \text { Gamma Scan, } \mathrm{DOH}(\mathrm{c})}\end{array}$ \\
\hline
\end{tabular}

(a) Refer to Figure 3.1, Food and Farm Product Sampl ing Locations.

(b) Three samples of each type collected within each area.

(c) Duplicate samples with the Washington State Department of Health. 


\subsection{WHEAT AND ALFALFA}

\begin{tabular}{|c|c|c|c|c|c|}
\hline Location (a) & $\begin{array}{c}\text { Sample } \\
\text { Iype }\end{array}$ & $\begin{array}{l}\text { Sample } \\
\text { Number }\end{array}$ & $\begin{array}{l}\text { Fre- }(b) \\
\text { quency }\end{array}$ & $\begin{array}{l}\text { Collection } \\
\text { Period } \\
\end{array}$ & Analyses \\
\hline East Wahluke Area & $\begin{array}{l}\text { Wheat } \\
\text { Alfalfa }\end{array}$ & $\begin{array}{l}0320 \\
0311\end{array}$ & $\begin{array}{l}B A \\
B A\end{array}$ & $\begin{array}{l}\text { July } \\
\text { May }\end{array}$ & $\begin{array}{l}90 \text { Sr, Gamma Scan } \\
90 \text { Sr, Garmma Scan }\end{array}$ \\
\hline Sagemoor Area & $\begin{array}{l}\text { Wheat } \\
\text { Alfalfa }\end{array}$ & $\begin{array}{l}0319 \\
0312\end{array}$ & $\hat{A}$ & $\begin{array}{l}\text { July } \\
\text { May }\end{array}$ & $\begin{array}{l}90 \mathrm{Sr}, \mathrm{Pu}(\mathrm{c}), \text { Garma Scan, FDA } \\
{ }^{90} \mathrm{Sr} \text {, Gamma Scan }\end{array}$ \\
\hline Riverview Area & $\begin{array}{l}\text { Wheat } \\
\text { Alfalfa }\end{array}$ & $\begin{array}{l}0318 \\
0313\end{array}$ & $\hat{A}$ & $\begin{array}{l}\text { July } \\
\text { May }\end{array}$ & $\begin{array}{l}90 \mathrm{Sr} \text {, Gamma Scan } \\
90 \mathrm{Sr} \text {, Gamma Scan, } \mathrm{DOH}^{(e)}\end{array}$ \\
\hline Benton City Area & $\begin{array}{l}\text { Wheat } \\
\text { Alfalfa }\end{array}$ & $\begin{array}{l}0317 \\
0314\end{array}$ & $\begin{array}{l}A \\
A\end{array}$ & $\begin{array}{l}\text { July } \\
\text { May }\end{array}$ & $\begin{array}{l}90 \mathrm{Sr} \text {, Gamma Scan } \\
90 \mathrm{Sr} \text {, Gamma Scan }\end{array}$ \\
\hline Sunnyside Area & $\begin{array}{l}\text { Wheat } \\
\text { Alfalfa }\end{array}$ & $\begin{array}{l}0316 \\
0315\end{array}$ & $\begin{array}{l}A \\
A\end{array}$ & $\begin{array}{l}\text { July } \\
\text { May }\end{array}$ & $\begin{array}{l}{ }^{90} \mathrm{Sr}, \mathrm{Pu}(\mathrm{c}), \text { Gamma Scan, } \mathrm{DOH}^{(\mathrm{e})} \\
{ }^{90} \mathrm{Sr} \text {, Gamma Scan, DOH }\end{array}$ \\
\hline Battelle Field & Alfalfa & 0301 & A & May & ${ }^{90} \mathrm{Sr}$, Gamma Scan, $\mathrm{DOH}^{(e)}$ \\
\hline Horn Rapids & Alfalfa & 0339 & A & May & ${ }^{90} \mathrm{Sr}$, Gamma Scan, $\mathrm{DOH}(\mathrm{e})$ \\
\hline
\end{tabular}

(a) Refer to Figure 3.1, Food and Farm Product Sampling Locations.

(b) Three samples of each type collected within each area. Biannual samples will next be collected in 1992.

(c) I sotopic plutonium.

(d) Dupl icate samples sent to the U.S. Food and Drug Administration.

(e) Duplicate samples with the Washington State Department of Heal th. 


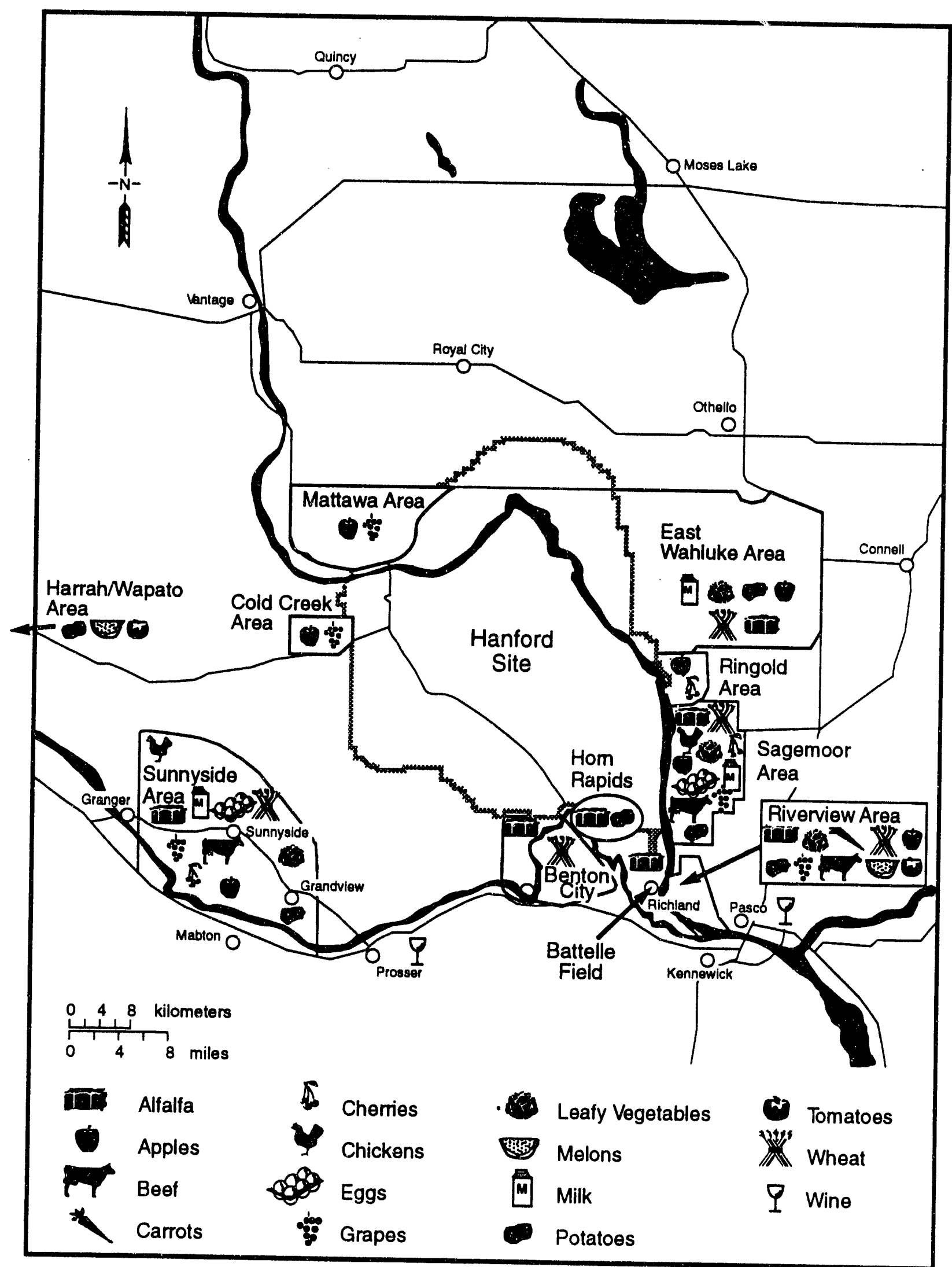

Figure 3.1. Food and Farm Product Sampling Locations

S9212076.6 


\subsection{WILDLIFE}

\subsection{AQUATIC BIOTA}

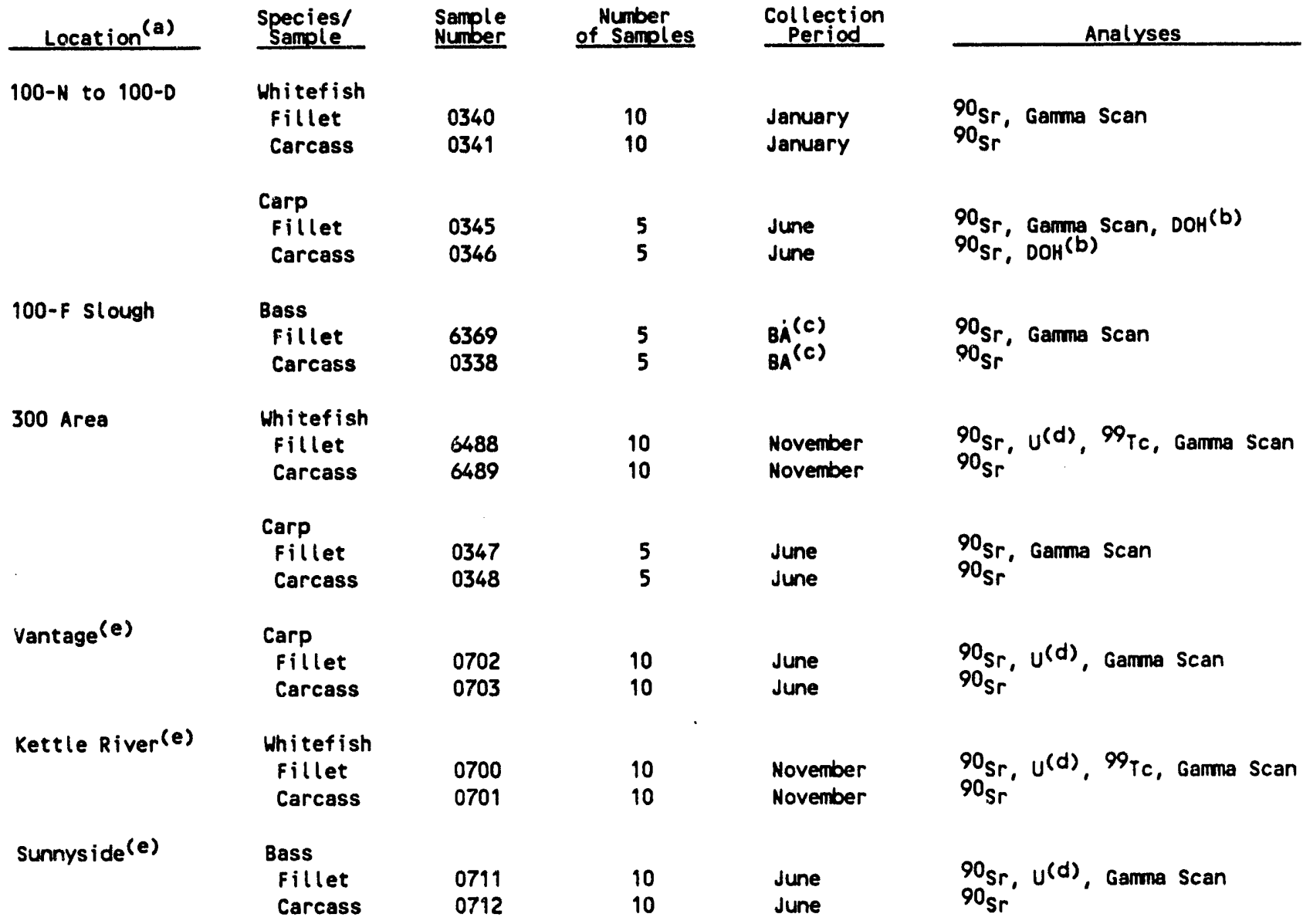

(a) Refer to Figure 4.1, Wildlife Sampling Areas.

(b) Dupl icate samples with the Hashington State Department of Heal th.

(c) Biannual samples will next be collected in 1994.

(d) Isotopic uranium.

(e) Background samples to be collected at least once every 5 years and will next be collected in 1995. 


\subsection{DUCKS}

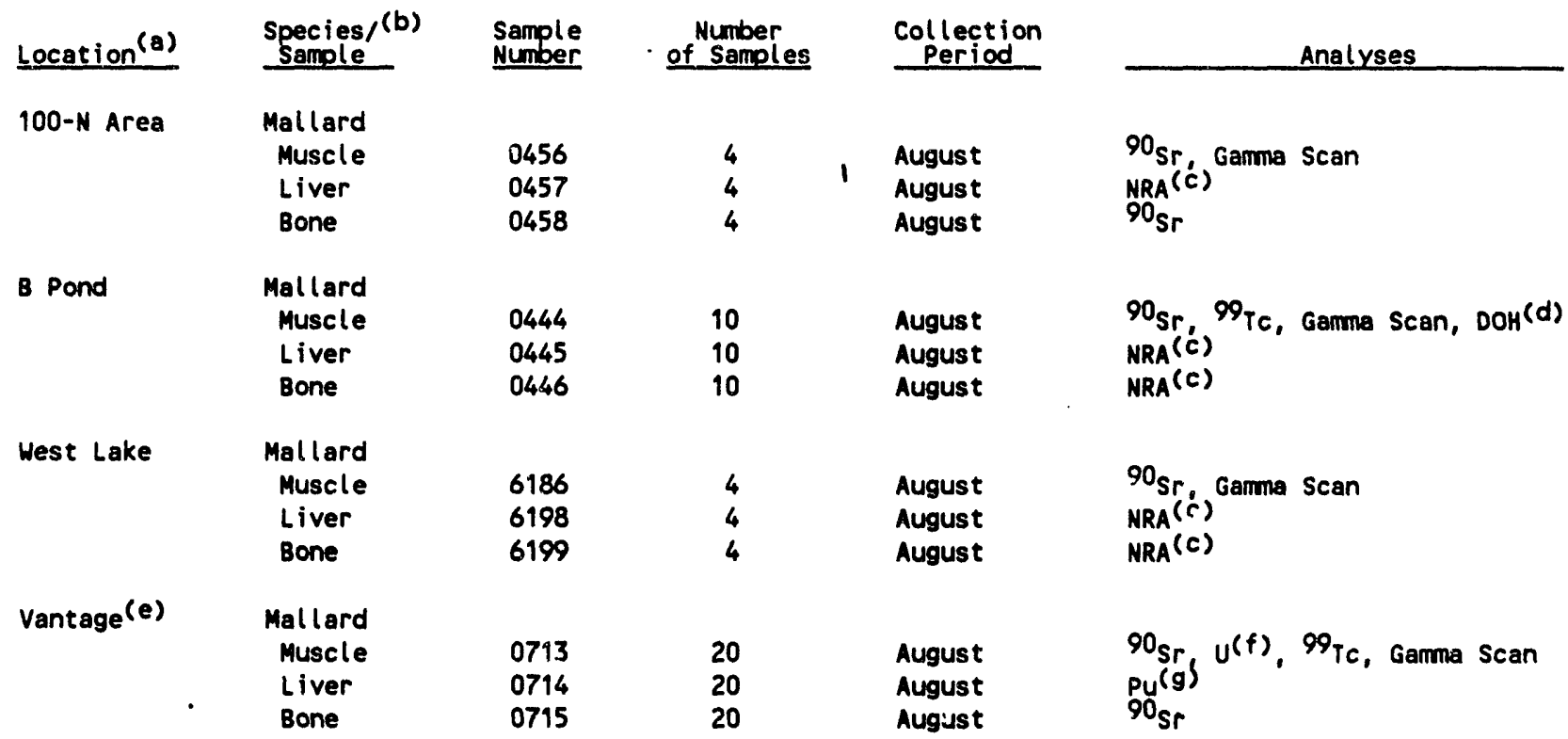

(a) Refer to Figure 4.1, Wildt ife Sampling Areas.

(b) Mallard preferred; other puddle ducks acceptable if mallard is unavailable.

(c) Not Routinely Analyzed (NRA) - Retain liver and bone to be analyzed for isotopic plutonium and $90 \mathrm{Sr}$, respectively, if $137 \mathrm{Cs}$ exceeds $100 \mathrm{pCi} / \mathrm{g}$ (wet weight) in the muscle.

(d) Dupl icaté samples with' the Washington State Department of Heal th.

(e) Background samples to be collected at least every 5 years and will next be collected in 1994 .

(f) Isotopic uranium.

(g) Isotopic plutonium.

\subsection{GAME BIRDS}

\begin{tabular}{|c|c|c|c|c|c|}
\hline Location (a) & $\begin{array}{l}\text { Species/(b) } \\
\text { Sample } \\
\end{array}$ & $\begin{array}{l}\text { Sample } \\
\text { Number }\end{array}$ & $\begin{array}{c}\text { Number } \\
\text { of Samples }\end{array}$ & $\begin{array}{c}\text { Collection } \\
\text { Period } \\
\end{array}$ & Analyses \\
\hline Near $100-N$ & $\begin{array}{l}\text { Pheasant } \\
\text { Muscle } \\
\text { Liver } \\
\text { Bone }\end{array}$ & $\begin{array}{l}0479 \\
0480 \\
0481\end{array}$ & $\begin{array}{l}3 \\
3 \\
3\end{array}$ & $\begin{array}{l}\text { January } \\
\text { January } \\
\text { January }\end{array}$ & $\begin{array}{l}{ }^{90} \mathrm{Sr}, \text { Gamma Scan } \\
\text { NRA }(\dot{C}) \\
{ }^{90}{ }_{\mathrm{Sr}}\end{array}$ \\
\hline $100-D$ to $100-H$ & $\begin{array}{l}\text { Pheasant } \\
\text { Muscle } \\
\text { Liver } \\
\text { Bone }\end{array}$ & $\begin{array}{l}0490 \\
0491 \\
0492\end{array}$ & $\begin{array}{l}4 \\
4 \\
4\end{array}$ & $\begin{array}{l}\text { January } \\
\text { January } \\
\text { January }\end{array}$ & $\begin{array}{l}\text { Gamma Scan } \\
\text { NRA (C) } \\
90_{S r}\end{array}$ \\
\hline $100-H$ to $100-F$ & $\begin{array}{l}\text { Pheasant } \\
\text { Muscle } \\
\text { Liver } \\
\text { Bone }\end{array}$ & $\begin{array}{l}0493 \\
0494 \\
0495\end{array}$ & $\begin{array}{l}6 \\
6 \\
6\end{array}$ & $\begin{array}{l}\text { January } \\
\text { January } \\
\text { January }\end{array}$ & $\begin{array}{l}\text { Gamma Scan, DOH } \\
\text { NRA }(c) \\
90_{\mathrm{Sr}}\end{array}$ \\
\hline Yakima County $(f)$ & $\begin{array}{l}\text { Pheasant } \\
\text { Muscle } \\
\text { Liver } \\
\text { Bone }\end{array}$ & $\begin{array}{l}0716 \\
0717 \\
0718\end{array}$ & $\begin{array}{l}20 \\
20 \\
20\end{array}$ & $\begin{array}{l}\text { September } \\
\text { September } \\
\text { September }\end{array}$ & $\begin{array}{l}{ }^{90} \mathrm{Sr} \\
\mathrm{Pu}_{\mathrm{Sg}} \mathrm{U}^{(\mathrm{e})} \text {, Gamma scan } \\
90_{\mathrm{Sr}}\end{array}$ \\
\hline
\end{tabular}

(a) Refer to Figure 4.1, Wildlife Sampling Areas.

(b) Pheasant preferred; chukar or quail acceptable if pheasant is unavailable.

(c) Not Routinely Analyzed (NRA) - Retain liver and bone to be analyzed for isotopic

plutonium and $90 \mathrm{Sr}$, respectively, if $137 \mathrm{Cs}$ exceeds $100 \mathrm{pCi} / \mathrm{g}$ (wet weight) in muscle.

(d) bupl icate samples with the Washington State Department of Health.

(a) Uuplicate samples

(f) Background samples to be collected at least every 5 years and will next be collected in 1994 .

(g) Isotopic plutonium. 


\subsection{DEER}

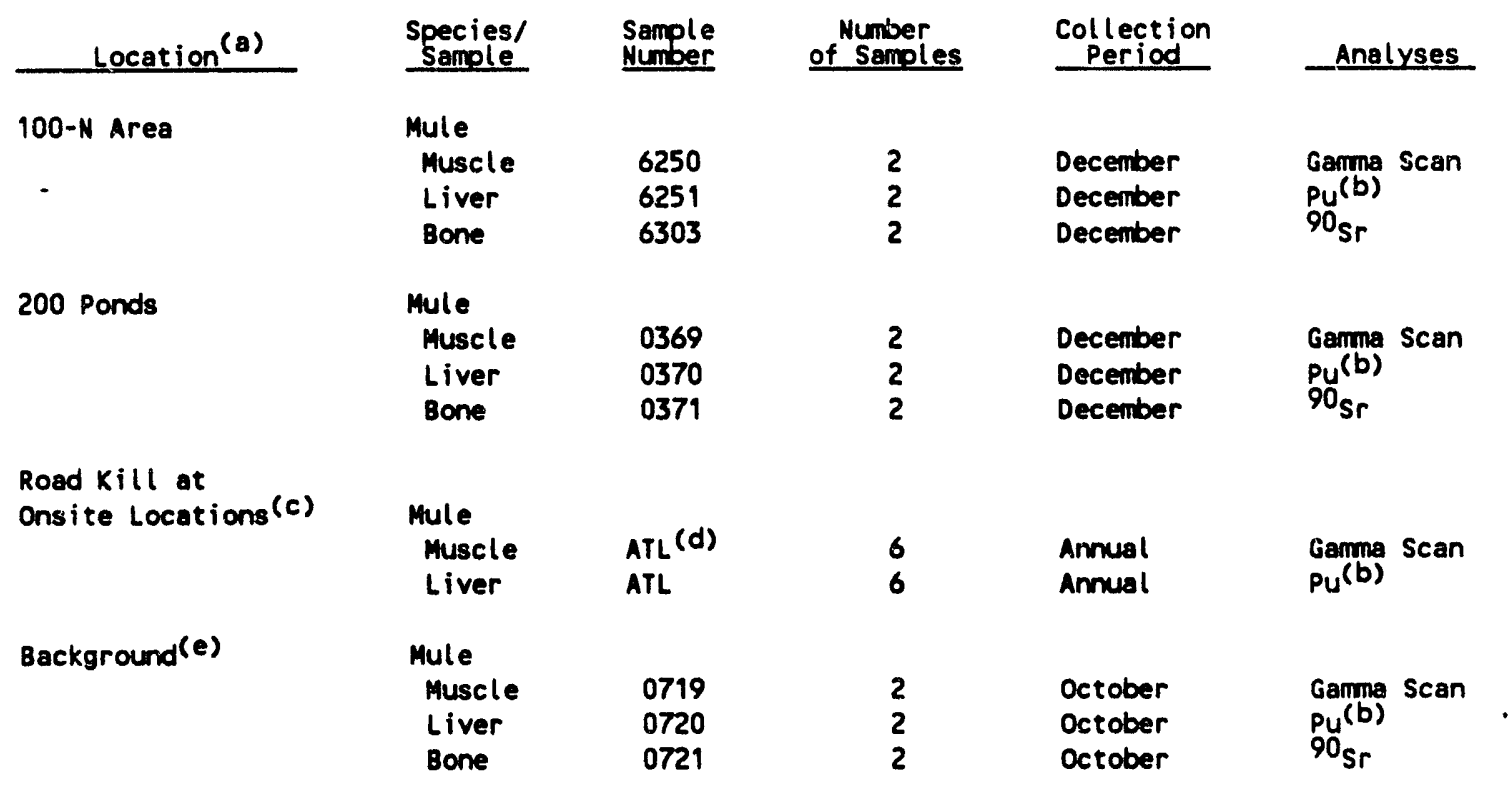

(a) Refer to Figure 4.1, Wildlife Sampling Areas.

(b) I sotopic plutonium.

(c) As available.

(d) ATL - according to location.

(e) Background samples to be coilected at least every 5 yesrs and will next be collected in 1995 .

\subsection{RABBITS}

\begin{tabular}{|c|c|c|c|c|c|}
\hline Location $(a)$ & $\begin{array}{l}\text { Type/ } \\
\text { Sample } \\
\end{array}$ & $\begin{array}{l}\text { Sample } \\
\text { Number }\end{array}$ & $\begin{array}{c}\text { Number } \\
\text { of Samples }\end{array}$ & $\begin{array}{l}\text { Collection } \\
\text { Period }\end{array}$ & Analyses \\
\hline $100-N$ Area & $\begin{array}{l}\text { Cottontail } \\
\text { Muscle } \\
\text { Liver } \\
\text { Bone }\end{array}$ & $\begin{array}{l}6145 \\
6146 \\
6452\end{array}$ & $\begin{array}{l}4 \\
4 \\
4\end{array}$ & $\begin{array}{l}\text { April } \\
\text { April } \\
\text { April }\end{array}$ & $\begin{array}{l}\text { Gamma Scan } \\
\mathrm{Pu}^{(\mathrm{b})} \\
{ }^{90} \mathrm{Sr}\end{array}$ \\
\hline 200-E Area & $\begin{array}{l}\text { Jack Rabbit } \\
\text { Muscle } \\
\text { Liver } \\
\text { Bone }\end{array}$ & $\begin{array}{l}1769 \\
1770 \\
6432\end{array}$ & $\begin{array}{l}4 \\
4 \\
4\end{array}$ & $\begin{array}{l}\text { April } \\
\text { April } \\
\text { April }\end{array}$ & $\begin{array}{l}\text { Gamma Scan } \\
P_{U}(b) \\
90_{S r}\end{array}$ \\
\hline 200-W Area & $\begin{array}{l}\text { Jack Rabbit } \\
\text { Muscle } \\
\text { Liver } \\
\text { Bone }\end{array}$ & $\begin{array}{l}1789 \\
1790 \\
6413\end{array}$ & $\begin{array}{l}4 \\
4 \\
4\end{array}$ & $\begin{array}{l}\text { April } \\
\text { April } \\
\text { April }\end{array}$ & $\begin{array}{l}\text { Gamma Scan } \\
\mathrm{Pu}(\mathrm{b}) \\
90_{\mathrm{Sr}}\end{array}$ \\
\hline N of 300 Area & $\begin{array}{l}\text { Cottontail } \\
\text { Muscle } \\
\text { Liver } \\
\text { Bone }\end{array}$ & $\begin{array}{l}6203 \\
6204 \\
6205\end{array}$ & $\begin{array}{l}4 \\
4 \\
4\end{array}$ & $\begin{array}{l}\text { April } \\
\text { April } \\
\text { April }\end{array}$ & $\begin{array}{l}\text { Gamma Scan } \\
\mathrm{Pu}^{(b)} \\
{ }^{90} \mathrm{Sr}\end{array}$ \\
\hline Boardman, $O R(C)$ & $\begin{array}{l}\text { Jack Rabbit } \\
\text { Muscle } \\
\text { Liver } \\
\text { Bone }\end{array}$ & $\begin{array}{l}0705 \\
0706 \\
0707\end{array}$ & $\begin{array}{l}10 \\
10 \\
10\end{array}$ & $\begin{array}{l}\text { April } \\
\text { April } \\
\text { April }\end{array}$ & $\begin{array}{l}\text { Gamma Scan } \\
\mathrm{Pu}^{(\mathrm{b})} \\
{ }^{0} \mathrm{O}_{\mathrm{Sr}}\end{array}$ \\
\hline
\end{tabular}




\subsection{RABBITS (contd)}

\begin{tabular}{|c|c|c|c|c|c|}
\hline Location (a) & $\begin{array}{l}\text { Type/ } \\
\text { Sample } \\
\end{array}$ & $\begin{array}{l}\text { Sample } \\
\text { Number }\end{array}$ & $\begin{array}{c}\text { Number } \\
\text { of Samples }\end{array}$ & $\begin{array}{l}\text { Collection } \\
\text { Period } \\
\end{array}$ & Analyses \\
\hline Boarchnan, $O R(c)$ & $\begin{array}{l}\text { Cottontail } \\
\text { Muscle } \\
\text { Liver } \\
\text { Bone }\end{array}$ & $\begin{array}{l}0708 \\
0709 \\
0710\end{array}$ & $\begin{array}{l}10 \\
10 \\
10\end{array}$ & $\begin{array}{l}\text { April } \\
\text { April } \\
\text { April }\end{array}$ & $\begin{array}{l}\text { Gamma Scan } \\
\mathrm{Pu}^{(\mathrm{b})} \\
90_{\mathrm{Sr}}\end{array}$ \\
\hline
\end{tabular}

(a) Refer to Figure 4.1, Wildlife Sampling Areas.

(b) Isotopic plutonium.

(c) Background samples to be collected at least every 5 years and will next be collected in 1995. 


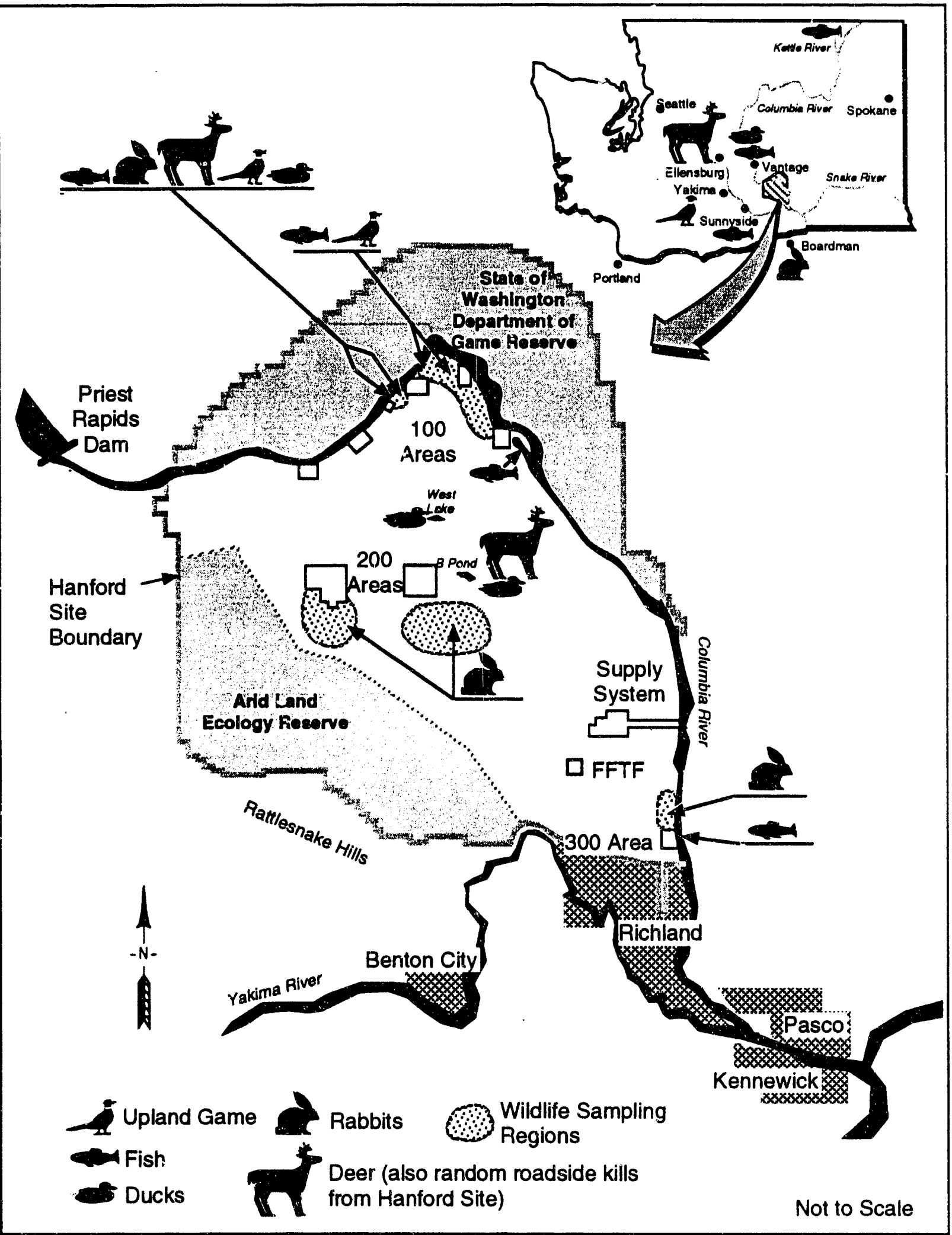

$\$ 9212076.7$

Figure 4.1 Wildlife Sampling Areas 


\subsection{SOIL AND VEGETATION}

\subsection{SOIL}

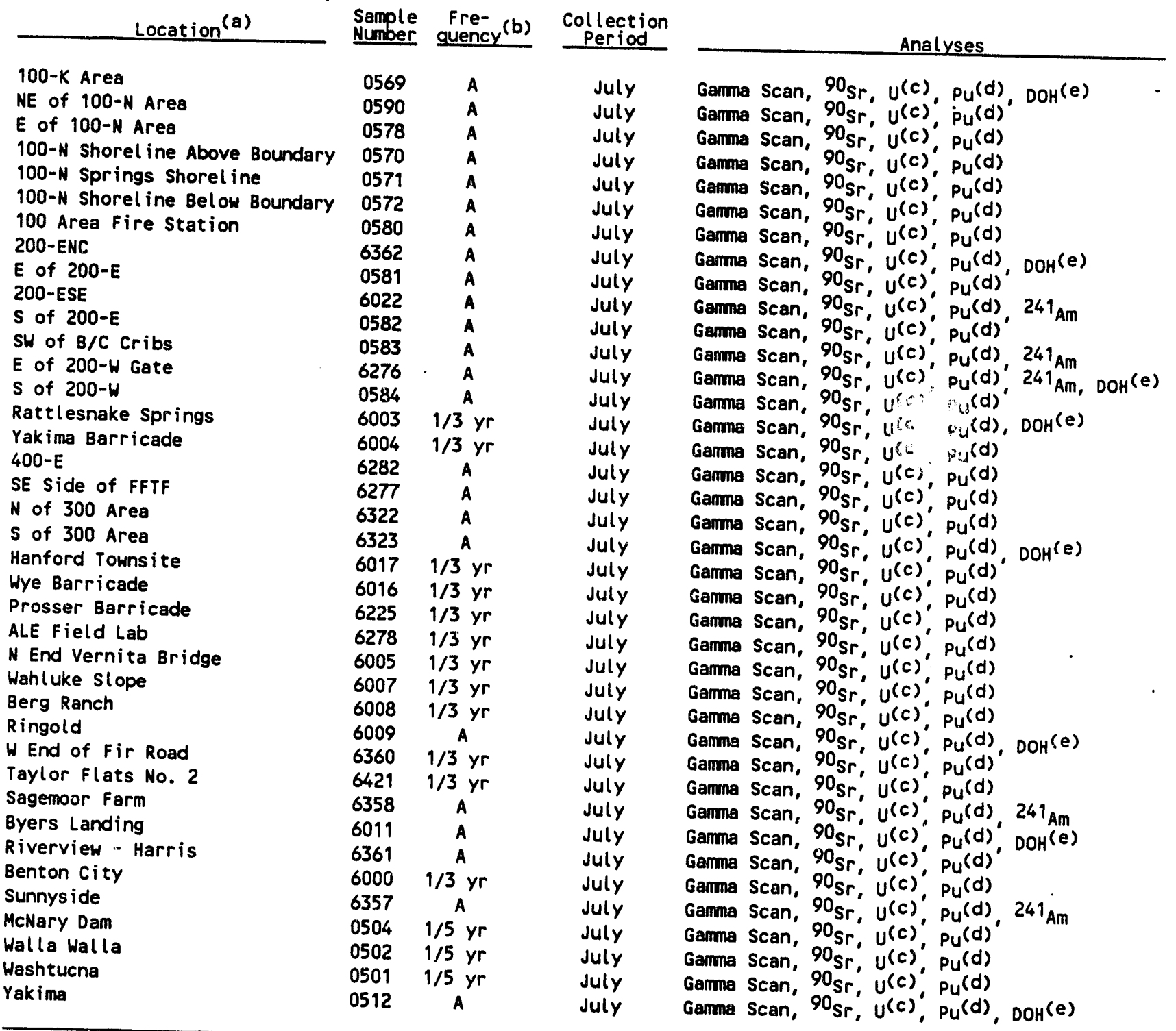

(a) Refer to figure 5.1, Soil and Vegetation Sampl ing Locations.

b) Specified samples are collected once every 3 or 5 years, respectively. The

(c) 235 U-LEP, $238 U$-LEP.

(d) Isotopic plutonium.

(e) Duplicate samples with the Washington State Department of Health. 


\subsection{VEGETATION}

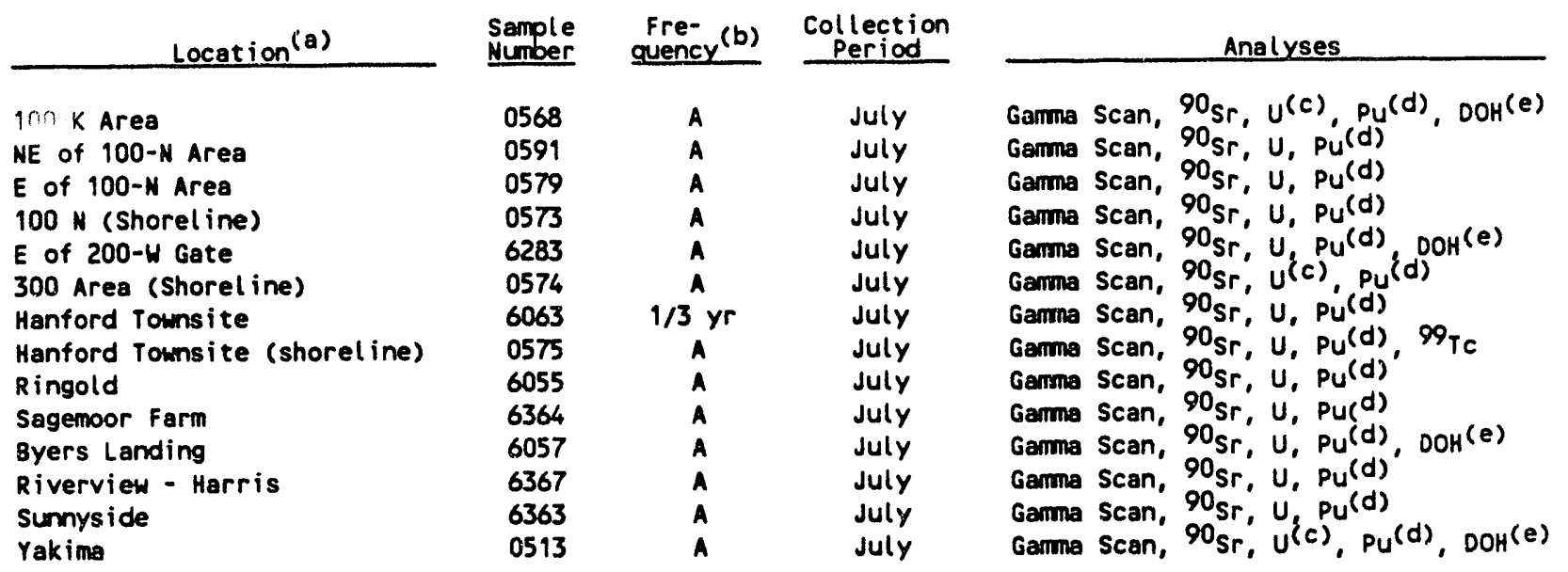

(a) Refer to Figure 5.1, Soil and Vegetation Sampling Locations.

(b) Specified samples are collected once every 3 years (1/3 yr). The next sampling will be 1993.

(c) Isotopic uranium.

(d) Isoropic plutonium.

(e) Duplicate samples with the Washington State Department of Health. 


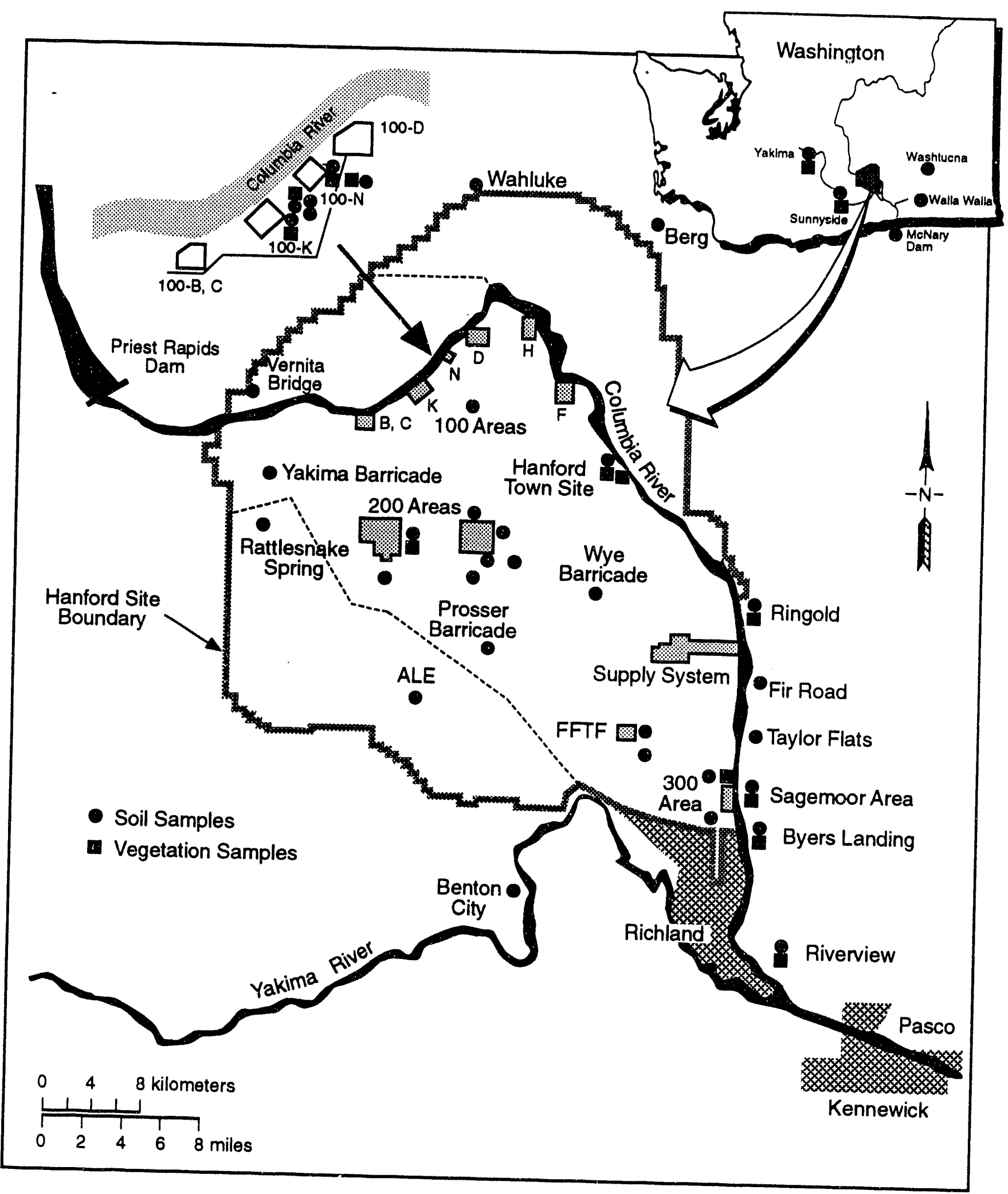

59212076.8

Figure 5.1. Soil and Vegetation Sampling Locations 


\subsection{SEDIMENT}

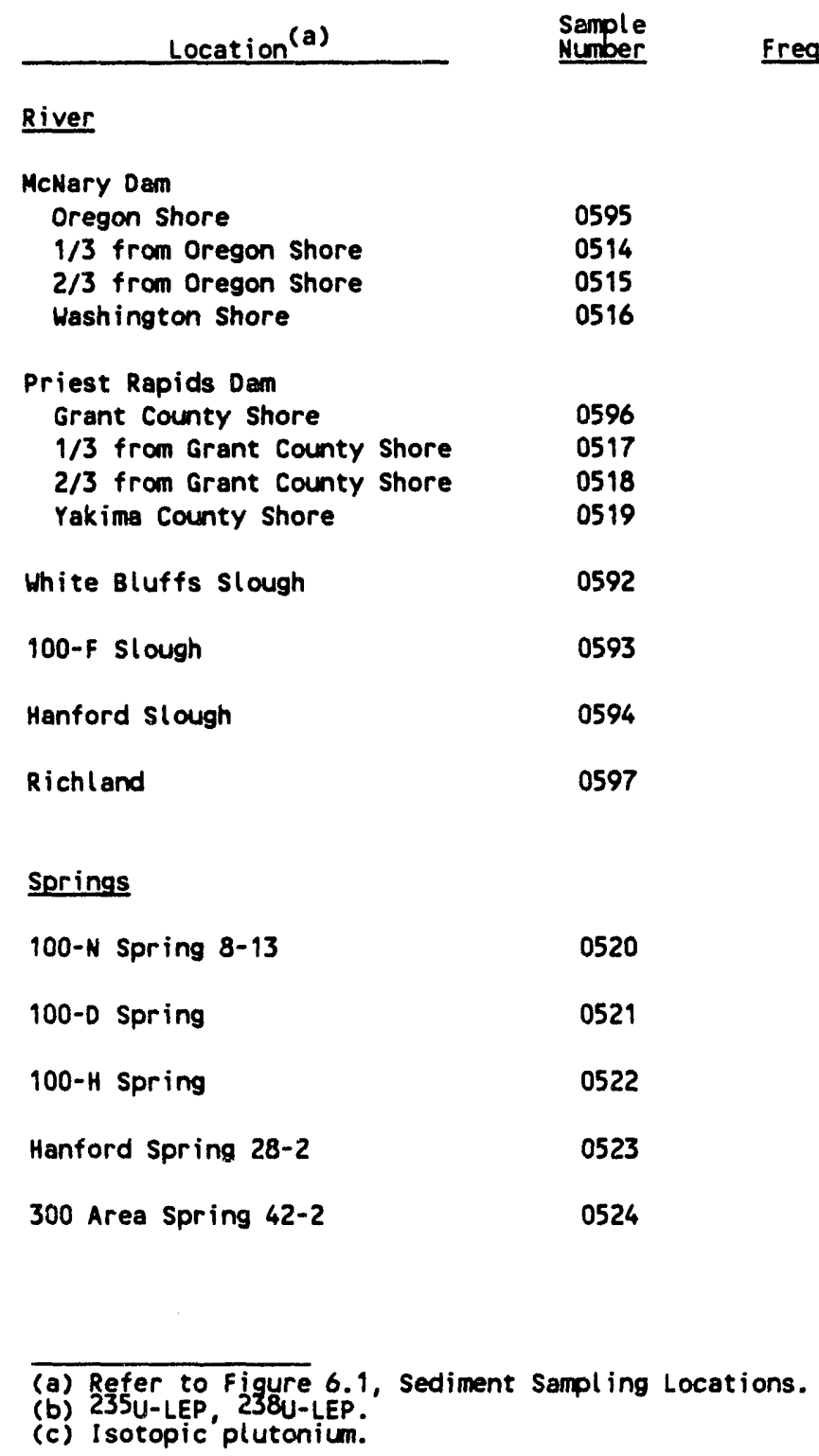

$\hat{A}$

A

A

A

A

A

A

A

A

Gamma Scan, ${ }^{90} \mathrm{Sr}, U(\mathrm{~b}), \mathrm{Pu}(\mathrm{c})$, ICP Metals Gamma scan, ${ }^{90} \mathrm{Sr}, \mathrm{u}^{(b)}, \mathrm{Pu}(\mathrm{c})^{\prime}$, ICP Metals Gamma Scan, $90 \mathrm{Sr}, u^{(b)}, \mathrm{Pu}(c)$, ICP Metals Gamma Scan, $90_{\mathrm{Sr}}, \mathrm{u}(b)^{\prime}, \mathrm{Pu}^{(c)}$, ICP Metals

Gamma Scan, $90 \mathrm{Sr}, \mathrm{u}^{(b)}, \mathrm{Pu}(\mathrm{c})$, ICP Metals Gamme Scan, ${ }^{90} \mathrm{Sr}, u^{(b)}, \mathrm{Pu}^{(c)}$, ICP Metals Gamma Scan, ${ }^{90} \mathrm{Sr}, u(b), P u(c)$, ICP Metals Gamma Scan, ${ }^{90} \mathrm{Sr}, \mathrm{u}(b)^{\prime}, \mathrm{Pu}^{(c)}$, ICP Metals

Gamma Scan, ${ }^{90} \mathrm{Sr}, \mathrm{u}^{(b)}, \mathrm{Pu}(\mathrm{c})$, ICP Metals Gamma Scan, ${ }^{90} \mathrm{Sr}, \mathrm{U}^{(b)}, \mathrm{Pu}(\mathrm{c})$, ICP Metals

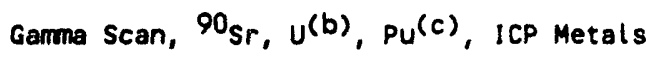
Gamma Scan, ${ }^{90} \mathrm{Sr}, \mathrm{u}^{(b)}, \mathrm{Pu}(\mathrm{c}), \mathrm{ICP}$ Metals

Gamma Scan, ${ }^{90} \mathrm{Sr}, \mathrm{U}^{(b)}$, ICP Metals

Gamma Scan, ${ }^{90} \mathrm{Sr}, \mathrm{u}^{(b)}$, ICP Metals

Gamma Scan, ${ }^{90} \mathrm{Sr}, \mathrm{U}^{(b)}$, ICP Metals

Gamma Scan, ${ }^{90} \mathrm{Sr}, \mathrm{u}^{(b)}$, ICP Metals

Gamma Scan, ${ }^{90} \mathrm{Sr}, \mathrm{u}(\mathrm{b})$, ICP Metals 


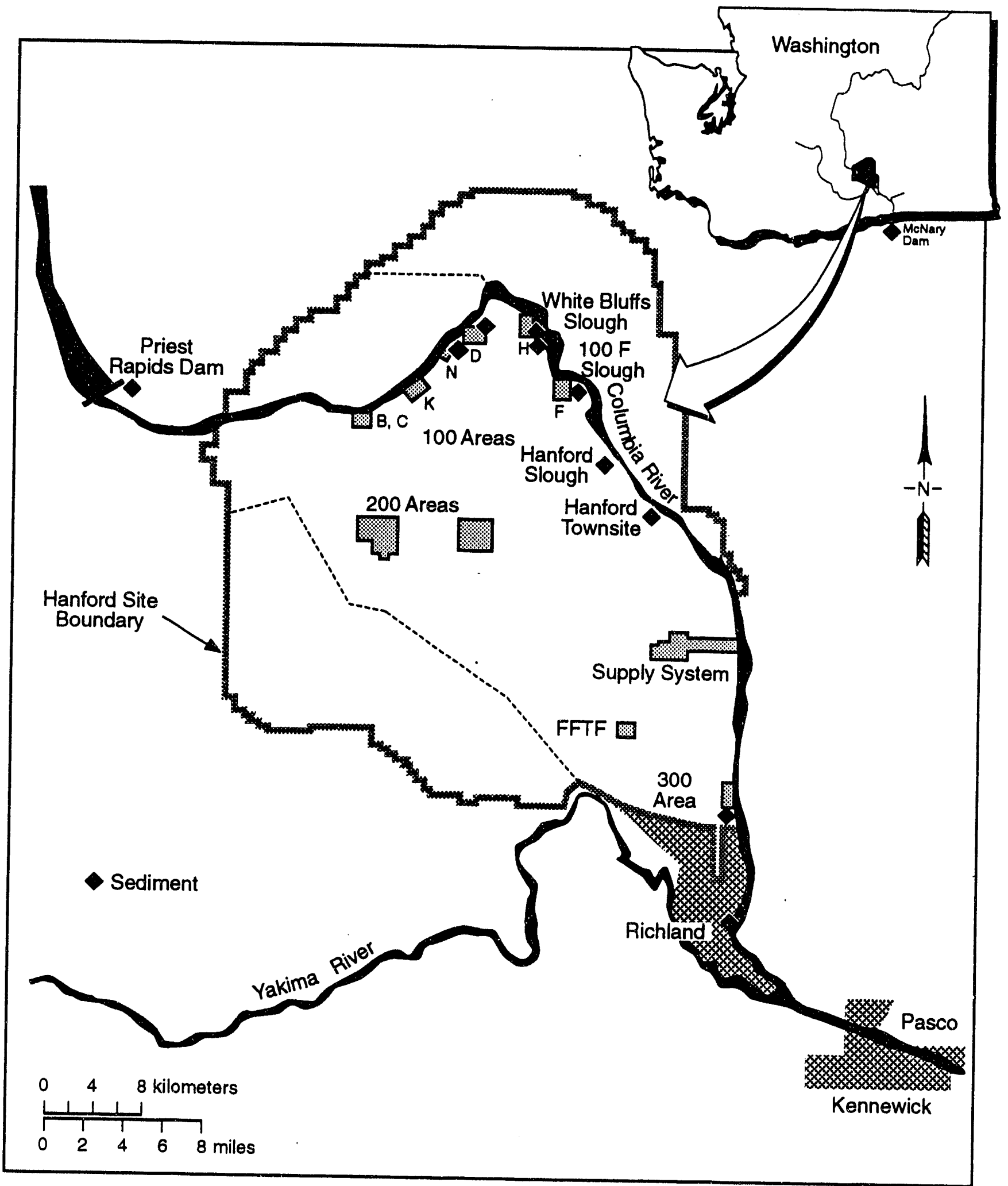

S9212076.10

Figure 6.1. Sediment Sampling Locations 


\subsection{EXTERNAL RADIATION}

\subsection{THERMOLUMINESCENT DOSIMETERS (TLDS)}

\subsubsection{Terrestrial Locations}

\begin{tabular}{|c|c|c|c|c|c|}
\hline Location & $\begin{array}{l}\text { Location } \\
\text { Number } \\
\end{array}$ & $\begin{array}{l}\text { Sample } \\
\text { Number }\end{array}$ & Erequency & Measurement & Instrument \\
\hline \multicolumn{6}{|l|}{ Onsite ${ }^{(a)}$} \\
\hline 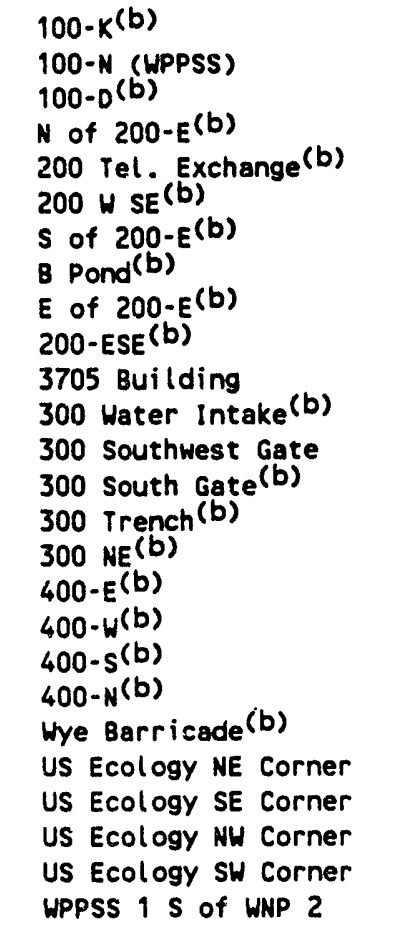 & $\begin{array}{r}1 \\
2 \\
3 \\
4 \\
5 \\
6 \\
7 \\
8 \\
9 \\
10 \\
11 \\
12 \\
13 \\
14 \\
15 \\
16 \\
17 \\
18 \\
19 \\
20 \\
21 \\
22 \\
23 \\
24 \\
25 \\
26\end{array}$ & $\begin{array}{l}1138 \\
1136 \\
1398 \\
1141 \\
1142 \\
1143 \\
1145 \\
1146 \\
1148 \\
1399 \\
1149 \\
1150 \\
1151 \\
1152 \\
1153 \\
1154 \\
1400 \\
1155 \\
1156 \\
1158 \\
1395 \\
0118 \\
0119 \\
0120 \\
0121 \\
0122\end{array}$ & $\begin{array}{l}0 \\
0 \\
0 \\
0 \\
0 \\
0 \\
0 \\
0 \\
0 \\
0 \\
0 \\
0 \\
0 \\
0 \\
0 \\
0 \\
0 \\
0 \\
0 \\
0 \\
0 \\
0 \\
0 \\
0 \\
0 \\
0 \\
0\end{array}$ & $\begin{array}{l}\text { Ambient Dose } \\
\text { Ambient Dose } \\
\text { Ambient Dose } \\
\text { Ambient Dose, } D O H(c) \\
\text { Ambient Dose, } D O H(c) \\
\text { Ambient Dose } \\
\text { Ambient Dose, } D O H(c) \\
\text { Ambient Dose } \\
\text { Ambient Dose, } D O H(c) \\
\text { Ambient Dose, } D O H(c) \\
\text { Ambient Dose } \\
\text { Ambient Dose } \\
\text { Ambient Dose } \\
\text { Ambient Dose } \\
\text { Ambient Dose } \\
\text { Ambient Dose } \\
\text { Ambient Dose } \\
\text { Ambient Dose } \\
\text { Ambient Dose } \\
\text { Ambient Dose } \\
\text { Ambient Dose, } D O H(c) \\
\text { Ambient Dose, } D O H(c) \\
\text { Ambient Dose, } D O H(c) \\
\text { Ambient Dose, } D O H(c) \\
\text { Ambient Dose, } D O H(c) \\
\text { Ambient Dose, } D O H(c)\end{array}$ & \\
\hline \multicolumn{6}{|l|}{ Perimeter ${ }^{(d)}$} \\
\hline $\begin{array}{l}\text { Ringold Met. Tower (b) } \\
\text { Byers Landing }(b) \\
\text { Battelle Complex }(b) \\
\text { WPPSS } 4 \text { WPPSS Warehouse } \\
\text { WPPSS } 8 \text { Fir Road }\end{array}$ & $\begin{array}{l}1 \\
2 \\
3 \\
4 \\
5\end{array}$ & $\begin{array}{l}0930 \\
1166 \\
1167 \\
0123 \\
0124\end{array}$ & $\begin{array}{l}0 \\
0 \\
0 \\
0 \\
0\end{array}$ & $\begin{array}{l}\text { Ambient Dose } \\
\text { Ambient Dose } \\
\text { Ambient Dose } \\
\text { Ambient Dose, } \mathrm{DOH}^{(\mathrm{c})} \\
\text { Ambient Dose, } \mathrm{DOH}^{(\mathrm{c})}\end{array}$ & . \\
\hline \multicolumn{6}{|l|}{ Community (d) } \\
\hline $\begin{array}{l}\text { Basin City(b)(e) } \\
\text { Pasco(b) } \\
\text { Kennewick(b) } \\
\text { Benton City(b) } \\
\text { Mattawa(b) } \\
\text { Edwin Markham }(b)(e) \\
\text { Richland }(L G P)(b)(e)\end{array}$ & $\begin{array}{r}6 \\
7 \\
8 \\
9 \\
10 \\
11 \\
12\end{array}$ & $\begin{array}{l}1936 \\
1378 \\
1379 \\
1381 \\
1384 \\
1921 \\
1906\end{array}$ & $\begin{array}{l}0 \\
0 \\
0 \\
0 \\
0 \\
0 \\
0\end{array}$ & $\begin{array}{l}\text { Ambient Dose } \\
\text { Ambient Dose } \\
\text { Ambient Dose } \\
\text { Ambient Dose } \\
\text { Ambient Dose } \\
\text { Ambient Dose } \\
\text { Ambient Dose }\end{array}$ & $\begin{array}{l}P I C^{(f)} \\
P I C^{(f)}\end{array}$ \\
\hline
\end{tabular}




\subsubsection{Terrestrial Locations (contd)}

\begin{tabular}{|c|c|c|c|c|c|}
\hline Location & $\begin{array}{l}\text { Location } \\
\text { Number } \\
\end{array}$ & $\begin{array}{l}\text { Sample } \\
\text { Number }\end{array}$ & Erequency & Measurement & Instrument \\
\hline Distant $(d)$ & & & & & \\
\hline $\begin{array}{l}\text { Sunnyside(b) } \\
\text { Yakima(b) }\end{array}$ & $\begin{array}{l}13 \\
14\end{array}$ & $\begin{array}{l}1388 \\
1389\end{array}$ & i & $\begin{array}{l}\text { Ambient Dose, } \mathrm{DOH}^{(c)} \\
\text { Ambient Dose, } \mathrm{DOH}^{(c)}\end{array}$ & \\
\hline
\end{tabular}

(a) Refer to Figure 7.1. Thermoluminescent Dosimeter (TLD) Locations on the Hanford Site.

(b) Located at air sampling station.

(c) Washington State Department of Heal th TLDs also at these locations. (c) Refer to Figure 7.2, Thermoluminescent Dosimeter (TLD) Locations for Perimeter and
Community sites.

(e) Cormunity-Operated Environmental Surveillance Station.

(f) PIC data (in $\mathrm{mR} / \mathrm{H}$ ) collected monthly. 


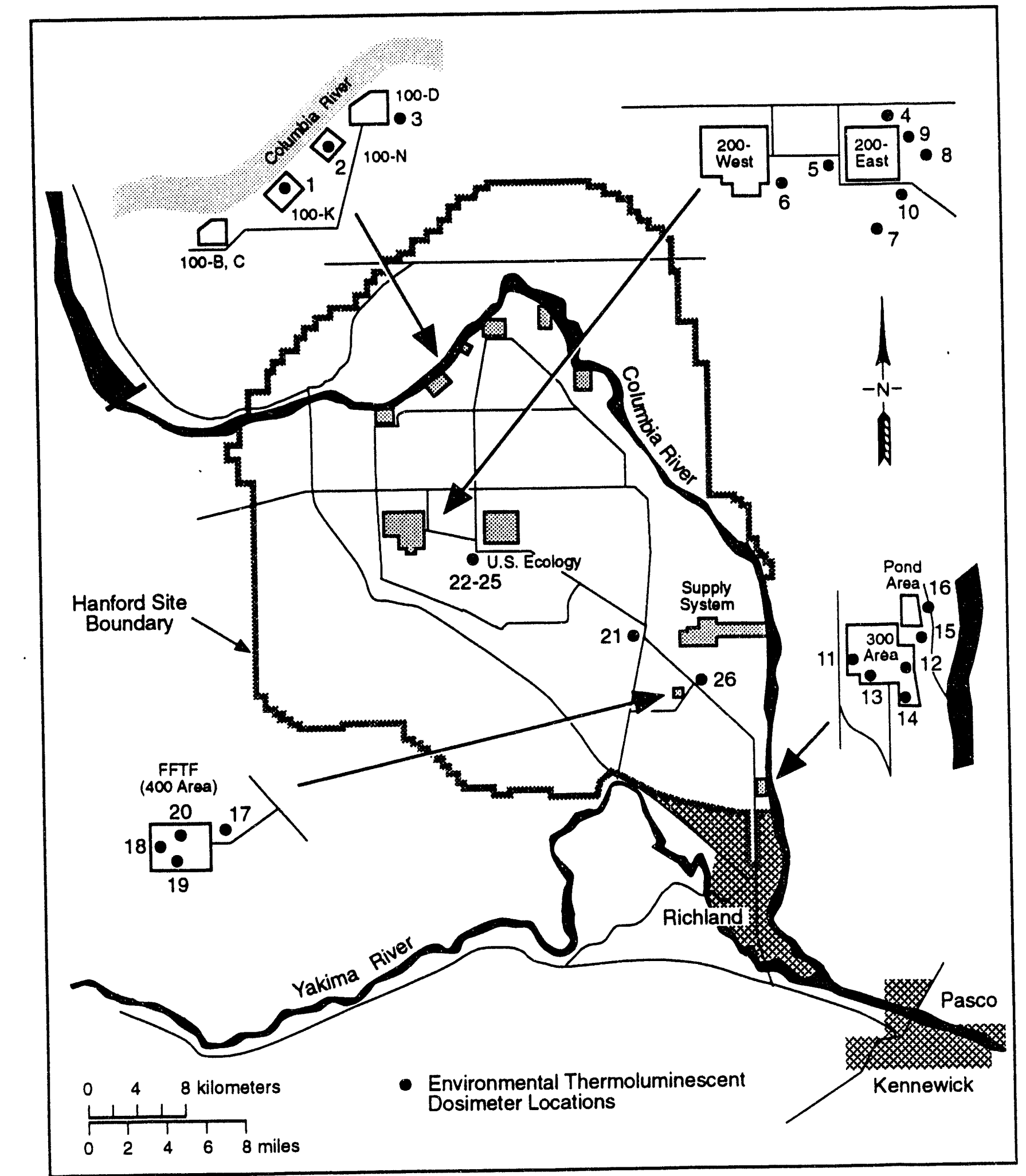

S9212076.3

Figure 7.1. Thermoluminescent Dosimeter (TLD) Locations on the Hanford Site 


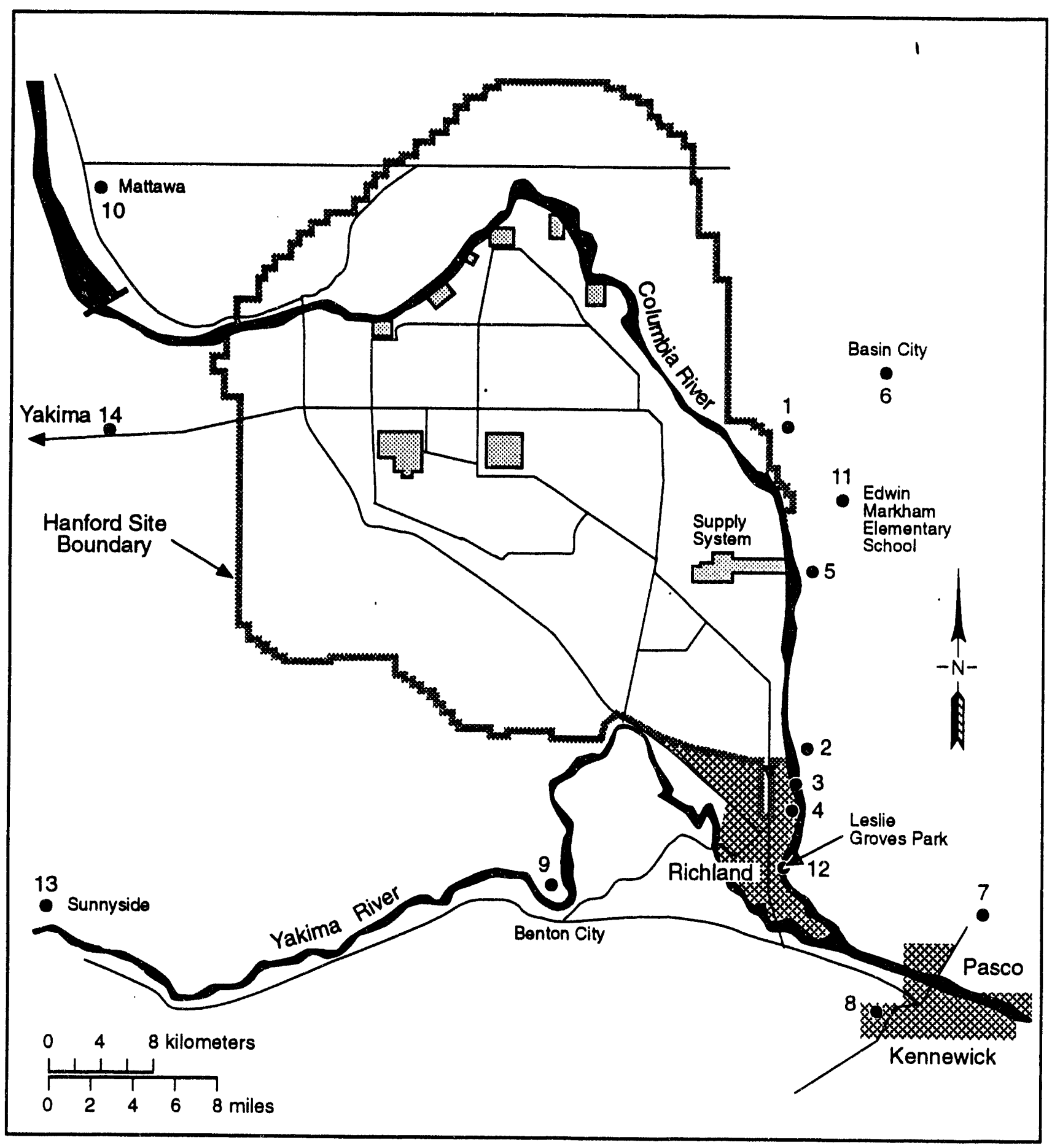

S9212076.1

Figure 7.2. Thermoluminescent Dosimeter (TLD) Locations for Perimeter and Community Sites 


\subsubsection{Columbia River Shoreline Locations}

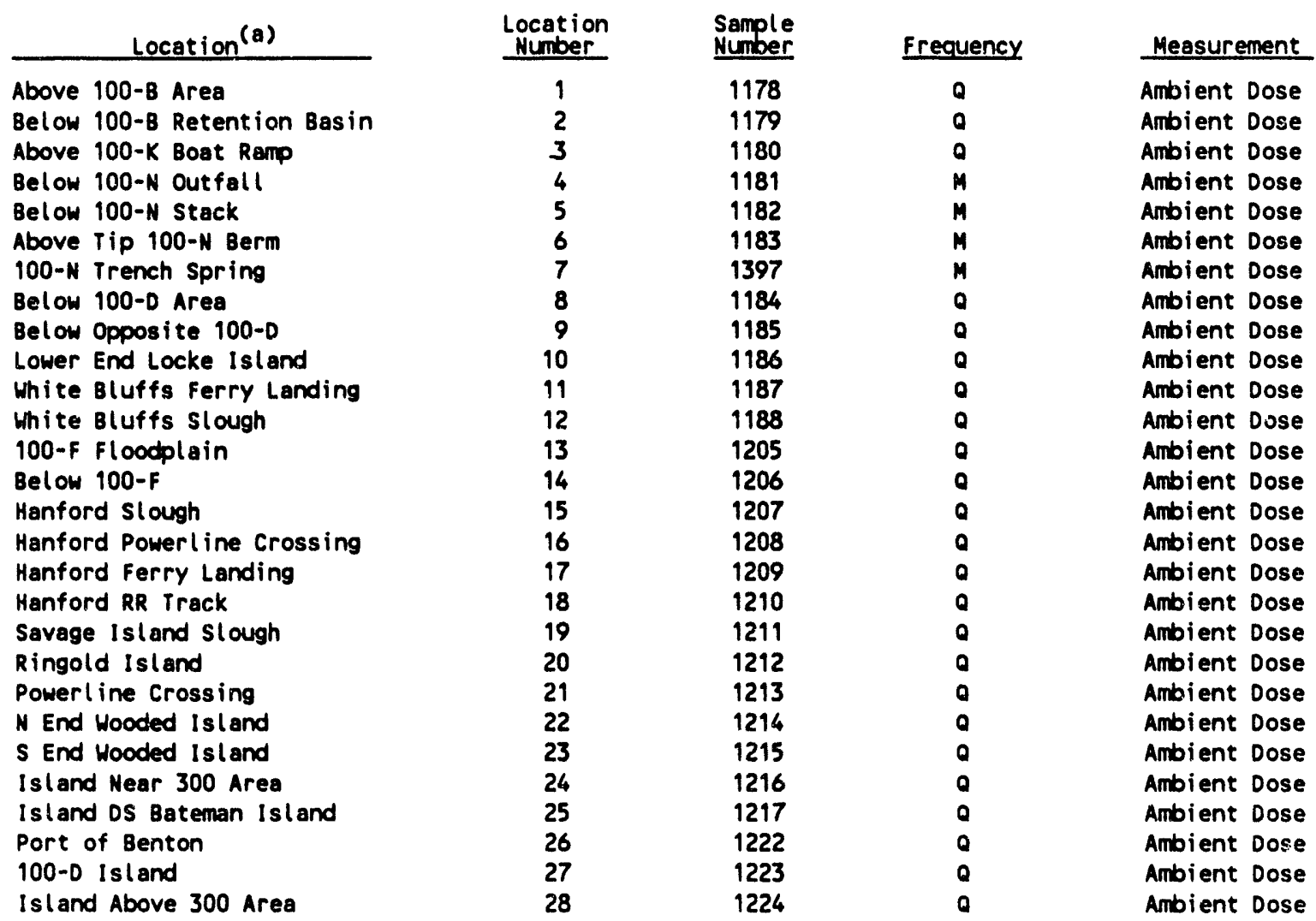

(a) Refer to Figure 7.3, Thermoluminescent Dosimeter (TLD) Locations on the Hanford Reach of the Columbia River. 


\subsection{COLUMBIA RIVER SHORELINE RADIATION SURVEYS}

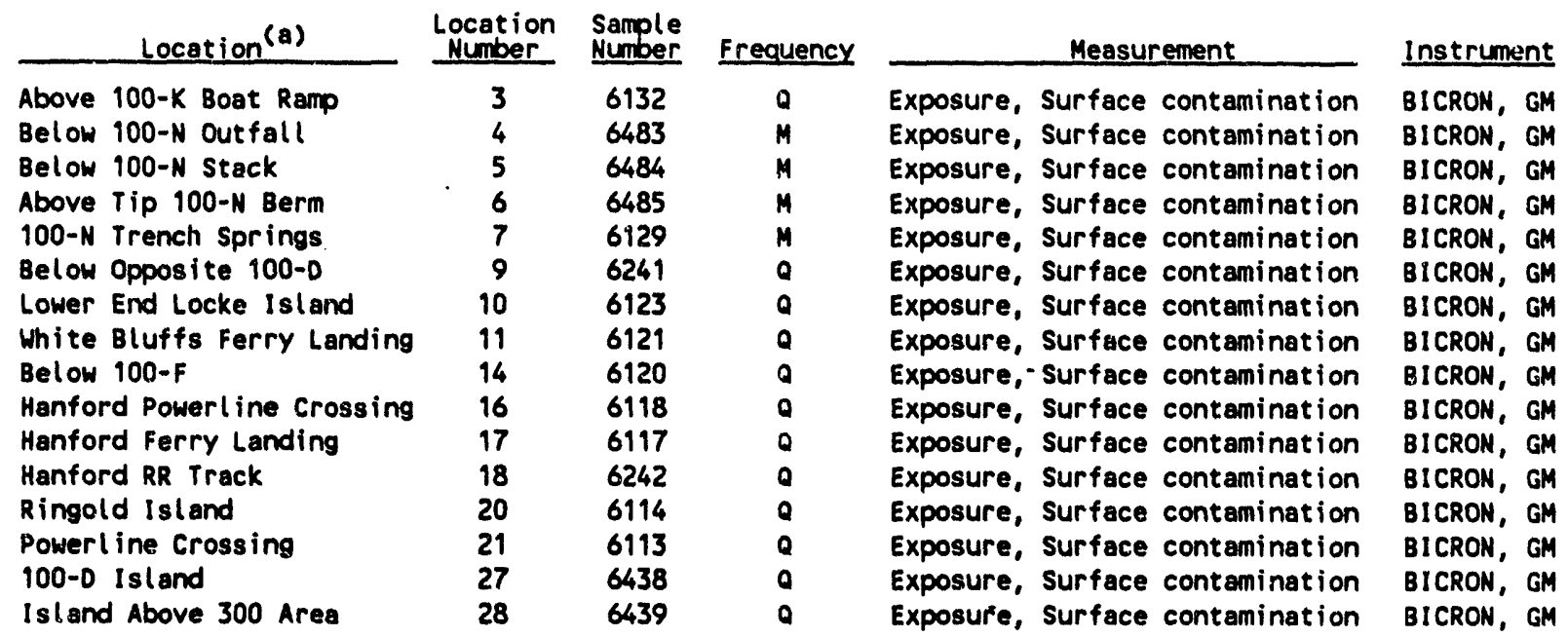

(a) Refer to Figure 7.3, Thermoluminescent Dosimeter (TLD) Locations on the Hanford Reach of the Columbia River. 


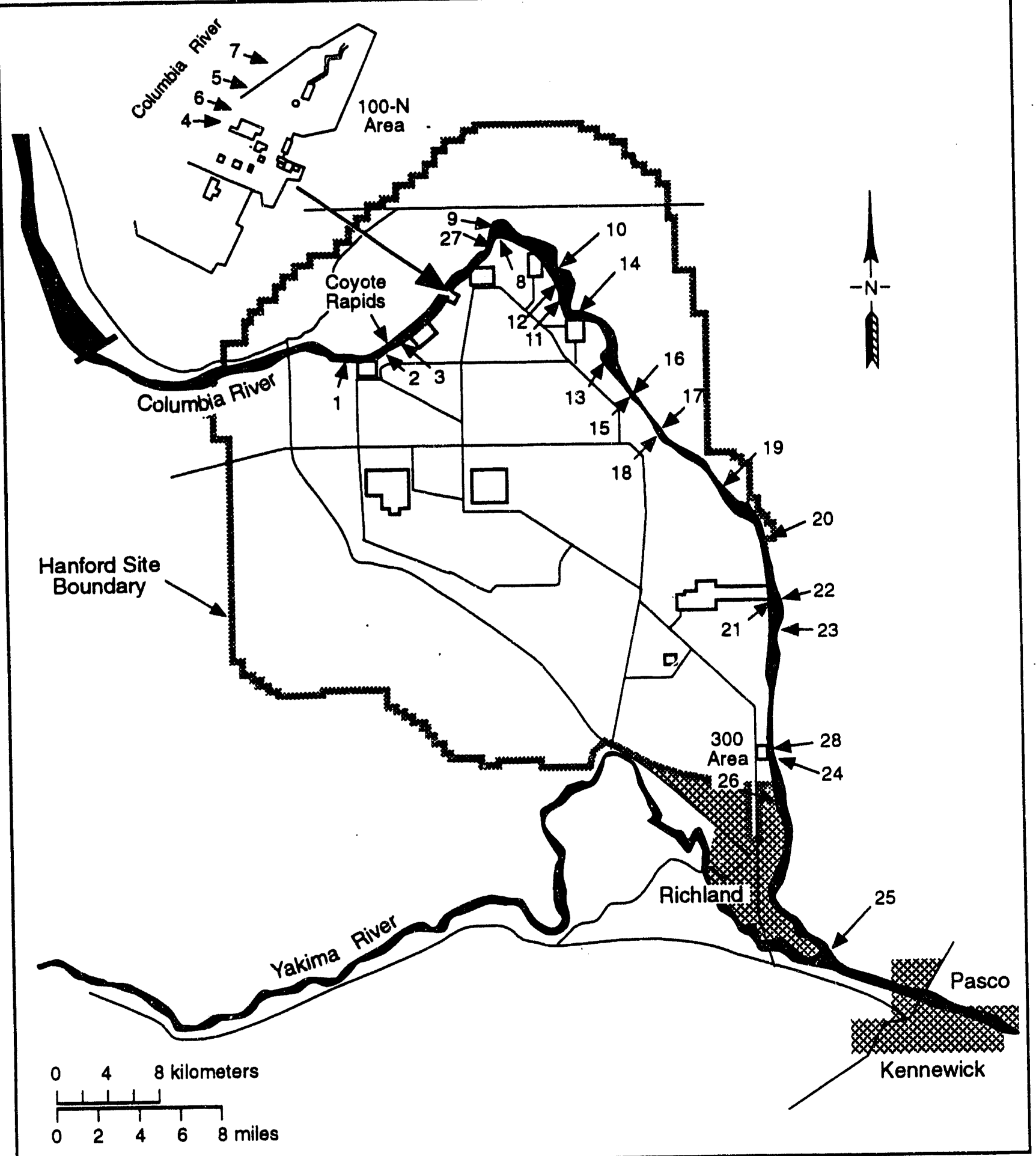

S9212076.2

Figure 7.3. Thermoluminescent Dosimeter (TLD) Locations on the Hanford Reach of the Columbia River 


\begin{tabular}{|c|c|c|c|c|}
\hline Description $^{(a)}$ & $\begin{array}{l}\text { Sample } \\
\text { Number }\end{array}$ & $\begin{array}{l}\text { Trip } \\
\text { Number } \\
\end{array}$ & Erequency & Instrument ${ }^{(b)}$ \\
\hline $\begin{array}{l}\text { 1100 Area to FFTF } \\
\text { 4-S at } 1100 \text { Area; 4-S at No. } 2 \text { RR Track; } \\
\text { 4-S at FFTF Access; FFTF; FFFF Access at 4-5; } \\
\text { 4-S at No. } 2 \text { RR Track; 4-S at } 300 \text { Area }\end{array}$ & 1800 & 1 & $M$ & Road Monitor \\
\hline $\begin{array}{l}\text { FFTF to US Ecology } \\
\text { 4-S at FFTF; } 4-5 \text { at Wye Barricade; } 4-5 \text { at } \\
\text { Army LoOp; } 4-5 \text { at US Ecology }\end{array}$ & 1801 & 2 & M & Road Monitor \\
\hline 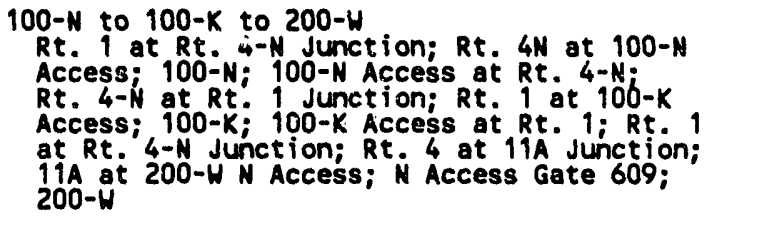 & 1802 & 3 & 0 & Road Monitor \\
\hline $\begin{array}{l}\text { Yakima Barricade to US Ecology } \\
\text { Yakima Barricade; ArmY Loop Road; 4-S; US } \\
\text { Ecology; 4-S Rt. 3; 200-W; 11A; 200-W; 4-S; } \\
\text { 11A }\end{array}$ & 1803 & 4 & $Q$ & Road Monitor \\
\hline $\begin{array}{l}\text { Army Loop Road } \\
\text { 4-s Army Loop; Old Powerl ine Crossing; } \\
\text { S 200-W Area; 11A Army Loop }\end{array}$ & 1804 & 5 & A & Road Monitor \\
\hline $\begin{array}{l}\text { US Ecology to FFTF } \\
\text { 4-S US Ecology; 4-S Army Loop; } \\
\text { Wye Barricade; Rt: 10 FFFF Access Road; } \\
\text { Prosser Barricade; Rt. 10 FFTF Access; } \\
\text { FFTF; Rt. } 10 \text { FFTF Access; Rt 10 and 4-s; } \\
\text { 4-S and FFTF Access Rood; FFTF }\end{array}$ & 1805 & 6 & A & Road Monitor \\
\hline $\begin{array}{l}\text { 300-Area through } 1100 \text { Area } \\
300 \text { Area; Garage; Stores; Gas Station; } \\
\text { Parking Lot; Ist street; Kaiser's Shop Area; } \\
\text { 1st street; IT; George Washington } \\
\text { Way - Stevens; IT }\end{array}$ & 1806 & 7 & A & Road Monitor \\
\hline 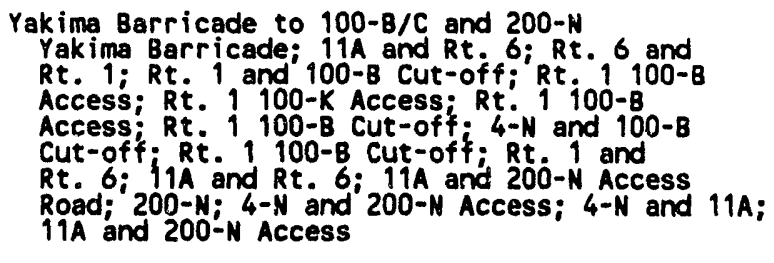 & 1807 & 8 & A & Road Monitor \\
\hline $\begin{array}{l}\text { Wye Barricade to } 100-N \\
\text { Hye Barricade; } 2-N \text { and } 11 A ; 2-N \text { and } 100-F ; \\
2-N \text { and } 100-H ; 2-N \text { and } 100-D ; 2-N \text { and } 4-N ; \\
4-N \text { and } 100-N A C c e s s ; 4-N \text { and } 2-N ; 2-N \text { and } \\
100-D ; 2-N \text { and } 100-H ; 2-N \text { and } 100-F ; 2-N \text { and } \\
11 A_{;} \text {iye Barricade }\end{array}$ & 1808 & 9 & $A$ & Road Monitor \\
\hline 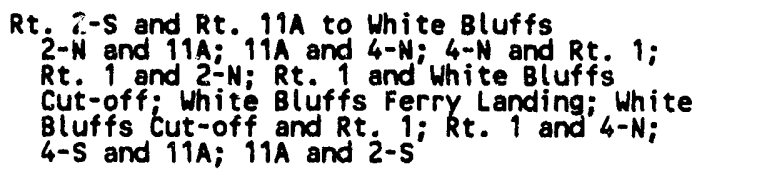 & 1809 & 10 & A & Road Monitor \\
\hline 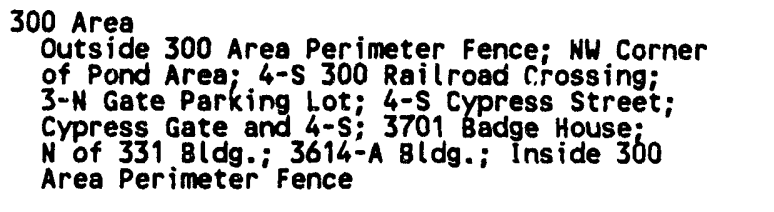 & 1810 & 11 & $Q$ & Road Monitor \\
\hline $\begin{array}{l}400 \text { Area } \\
\text { Visitor Center Access Road; Main Gate; NW } \\
\text { Corner Parking Lot; NE Corner Parking Lot; } \\
\text { Visitor Center; old 4-E Monitoring Station; } \\
\text { NE Corner Perimeter Fence; NH Corner Perimeter } \\
\text { Fence; SW Corner Perimeter Fence; SE Corner } \\
\text { Perimeter Fence; Main Gate }\end{array}$ & 1811 & 12 & 0 & Rosd Monitor \\
\hline
\end{tabular}

(a) Refer to Figure 7.4, Road Survey Routes.

(b) The instrument used for road monitoring consists of sodium iodide detectors with associated electronics which function as gross-gamme counters. 


\subsection{ONSITE RAILWAY RADIATION SURVEYS}

\begin{tabular}{|c|c|c|c|c|}
\hline 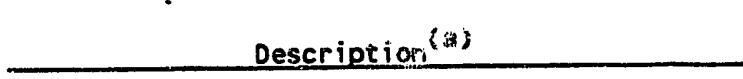 & $\begin{array}{l}\text { Sample } \\
\text { Number }\end{array}$ & $\begin{array}{c}\text { Trip } \\
\text { Number } \\
\end{array}$ & Frequency & Instrument ${ }^{(b)}$ \\
\hline $\begin{array}{l}1100 \text { Area; Ruby Junc:ion; } 300 \text { Area Gate; Geneva } \\
\text { Junction; FFf; May Junction; Willa Junction; } \\
\text { Peari Junction; Ethel Junction; } 200-\mathrm{E} \text { Gate; } \\
\text { Susie Junction; 200-W Gate }\end{array}$ & 1821 & 2 & 0 & Railway Monitor \\
\hline $\begin{array}{l}\text { May Junction; Ruth Junction; Ginger Junction } \\
\text { Betty Jurction; Nancy Junction; } 100-N \text { Gate; } \\
\text { Helen Junction; fudrey Junction; 100-K Gate; } \\
\text { Susie Junction(c) }\end{array}$ & 1822 & 3 & $Q$ & Railway Monitor \\
\hline $\begin{array}{l}1100 \text { Area; Van Giesfn; Yakima River Bridge; } \\
\text { Columbia Center }\end{array}$ & 1819 & 5 & a & Railway Monitor \\
\hline
\end{tabular}

(a) Refer to Figure 7.5 , Rail road Survey Routes. electronics which function as gross-gama counters.

(c) These surveys will be performed quarterly only if deemed necessary by the External Radiation Task Leader; at a minimm these will be performed annually.

\subsection{ONSITE AERIAL RADIATION SURVEYS}

$$
\text { Description }(a)
$$

Sample
Number

1820

$$
\text { Trip }
$$

3

Frizquency

A $e^{(b)}$

Aerial Monitor

(a) Survey is performed either annually or at an appropriate $f_{1}$ equicy determined for maintaining emergency preparedness support or post-accident characterization (currently the only purposes for it).

(b) Preparedness support or postal accident character used for aerial monitoring corisists of sodium iodide detectors with associated electronics which function as gross-gamma counters. 


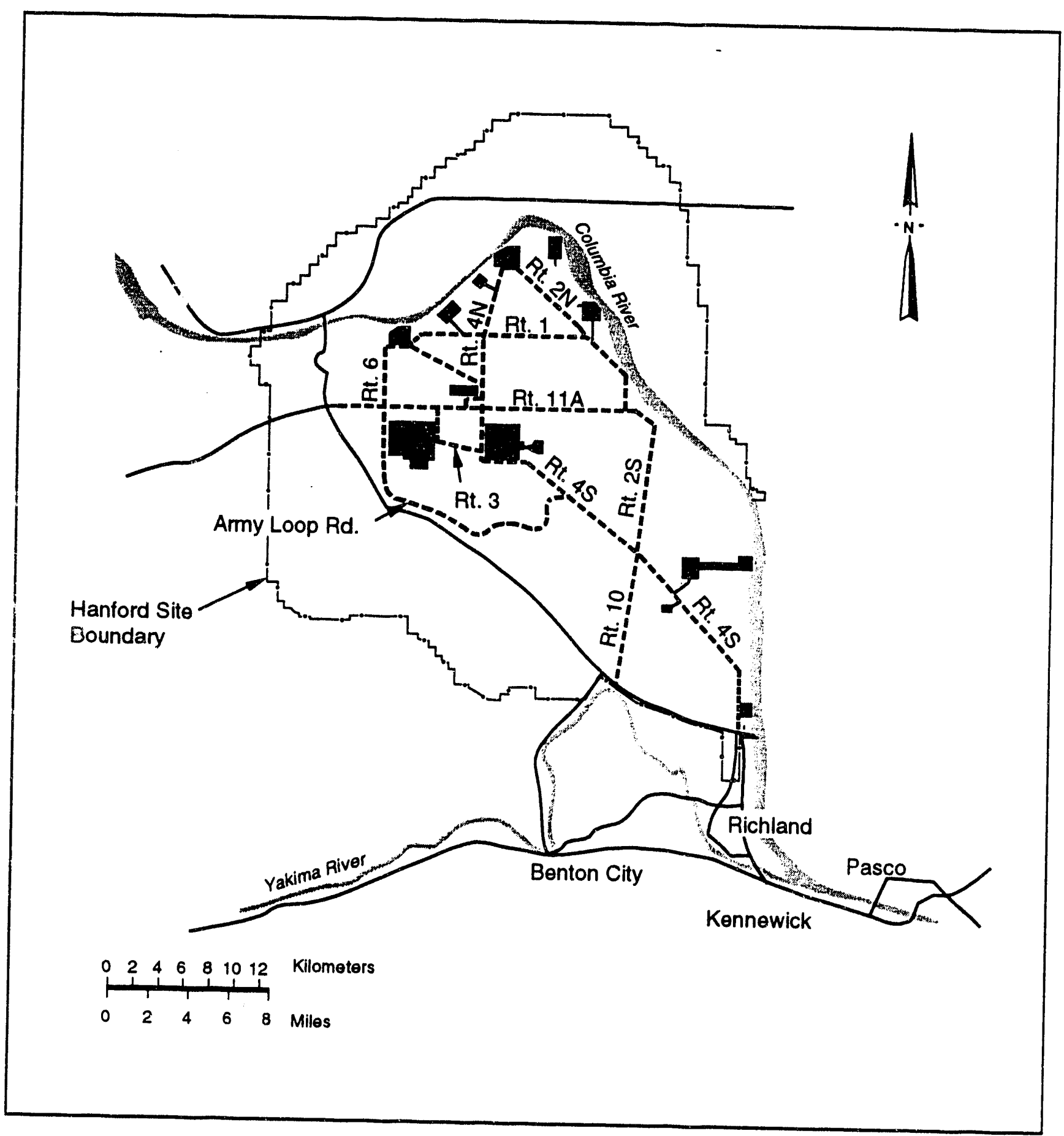

\$9108076.1

Figure 7.4. Road Survey Routes 


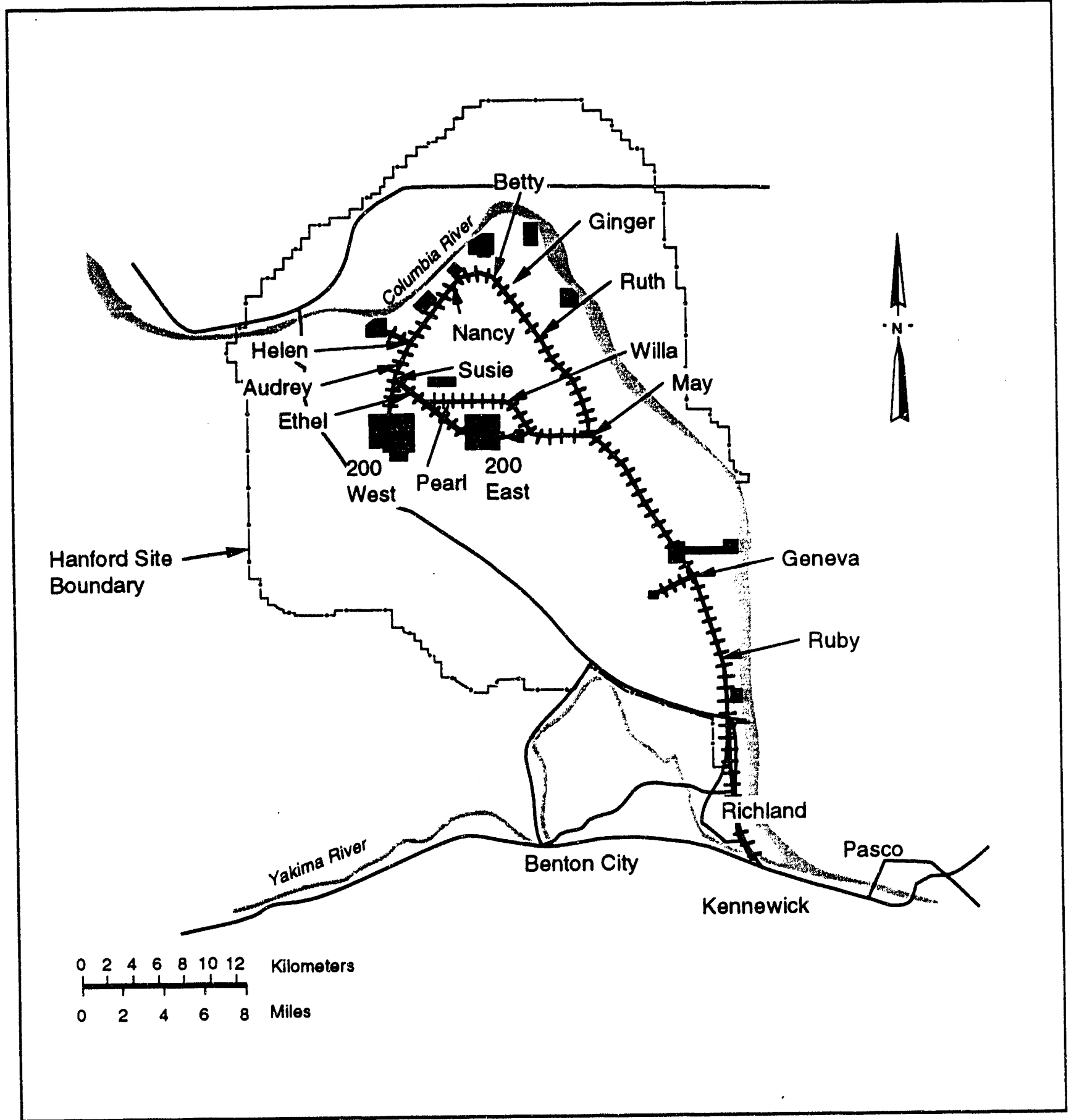

S9108076.2

Figure 7.5. Railroad Survey Routes 
PART II. HANFORD GROUND-WATER SURVEILLANCE 


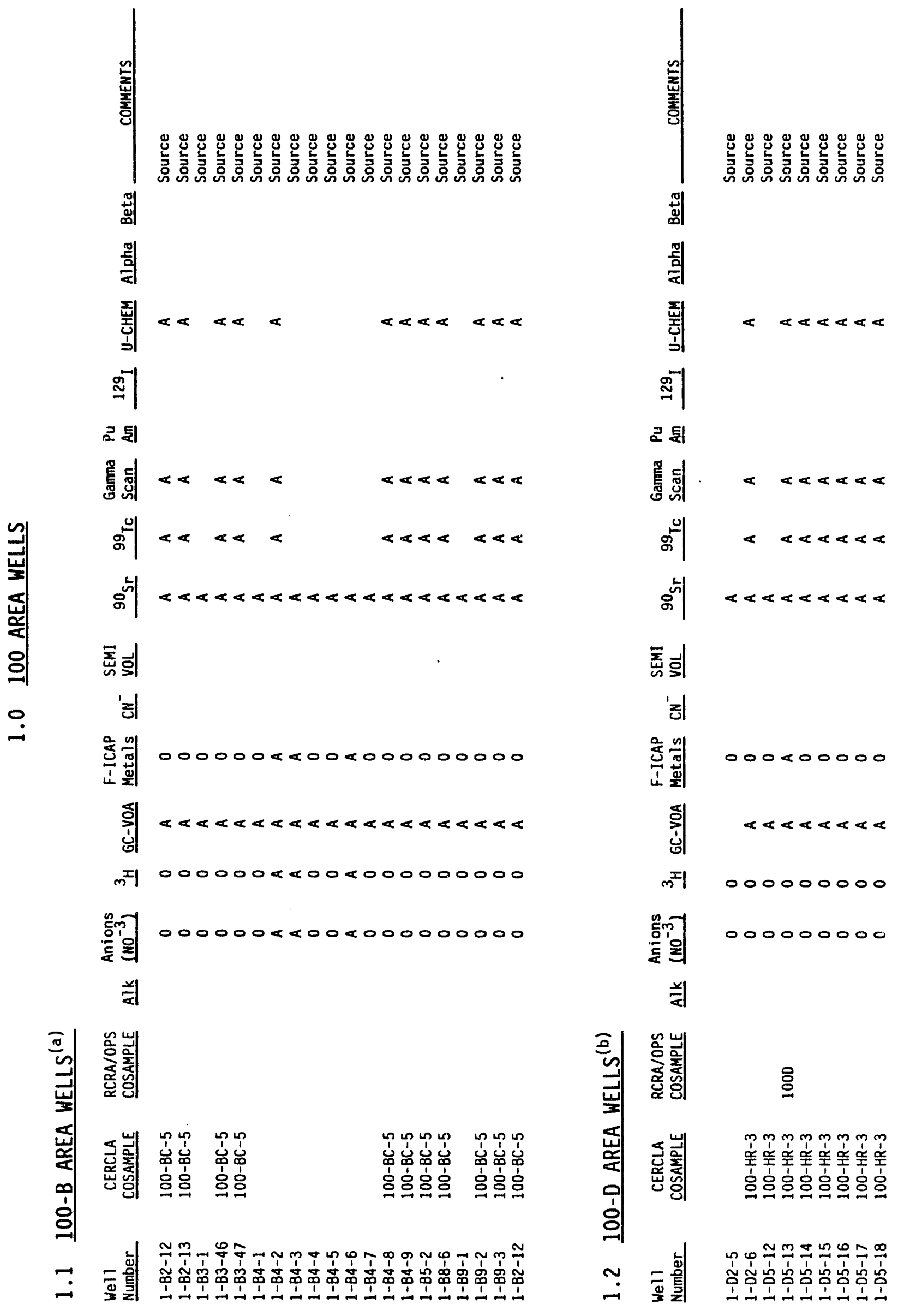




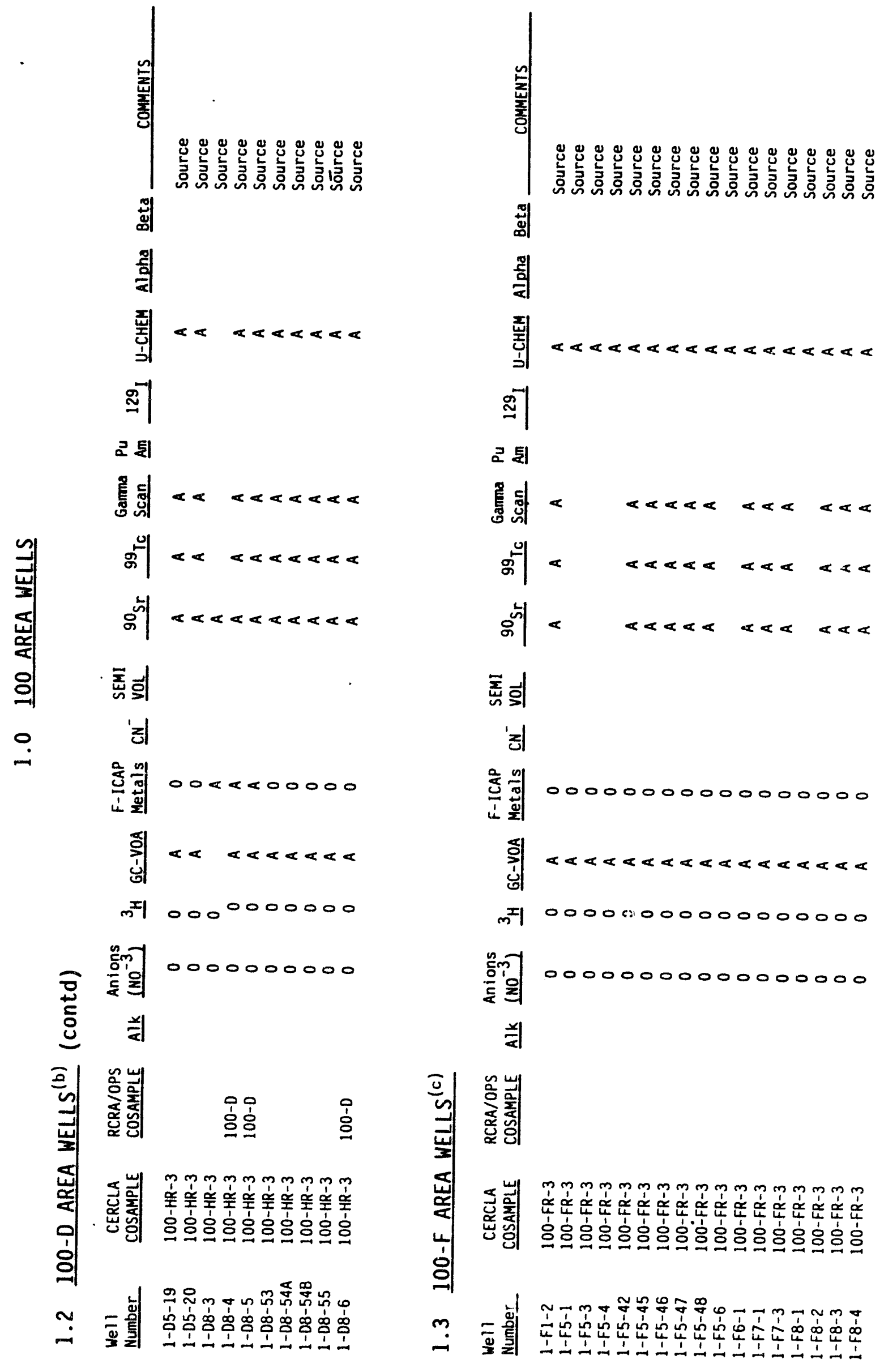




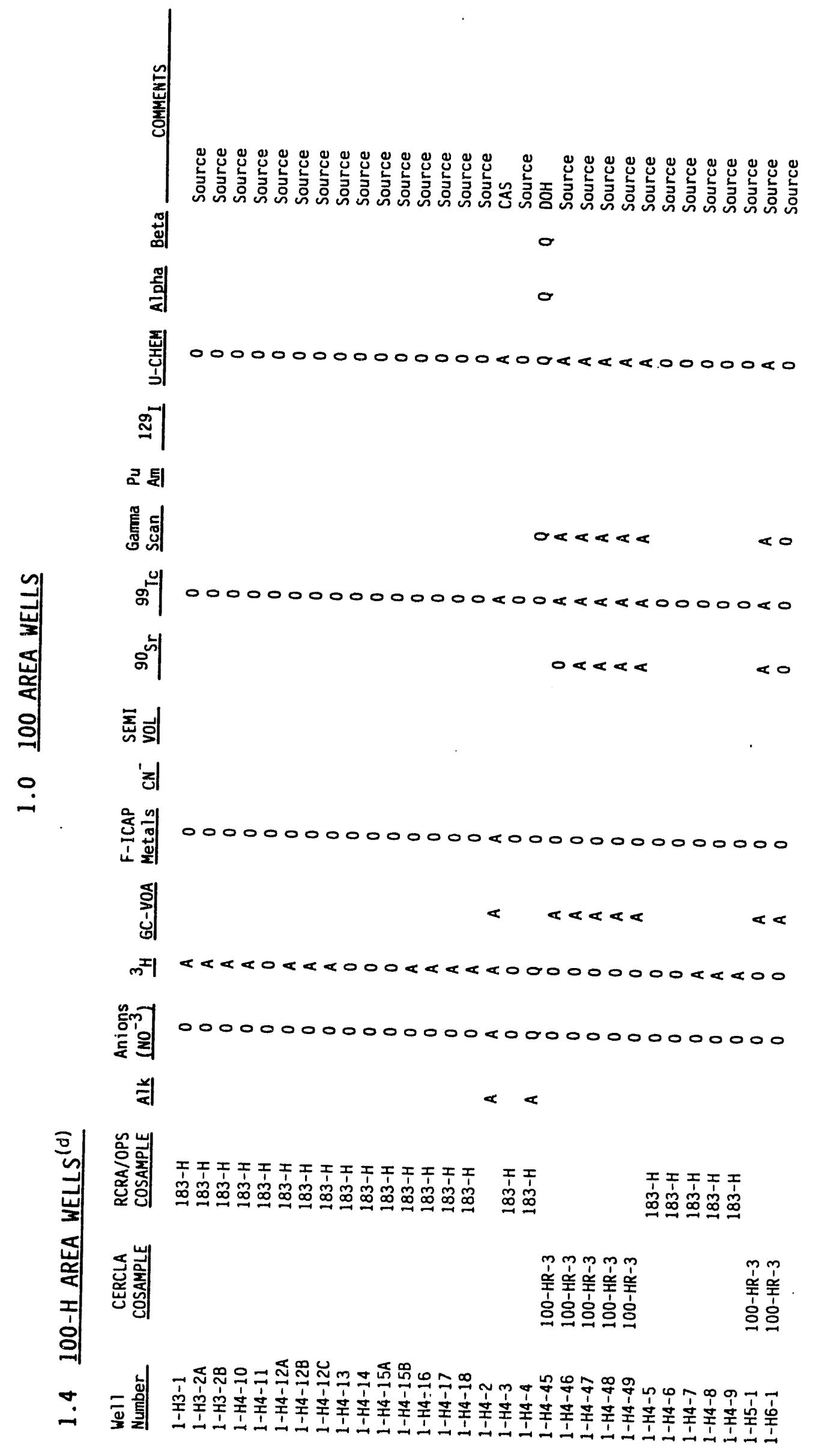




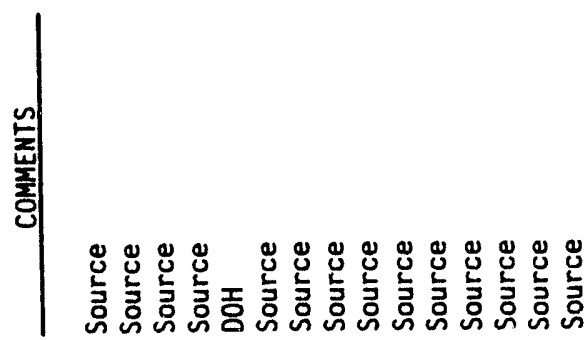

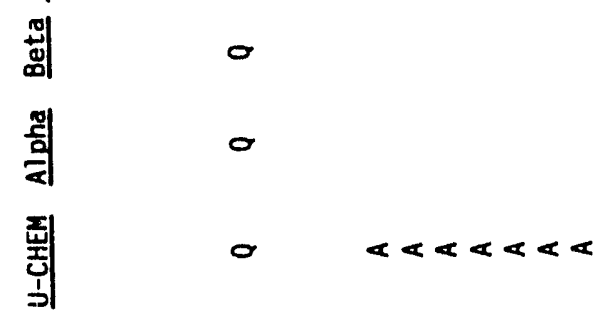

$\Xi$

로

总 웡

का

की

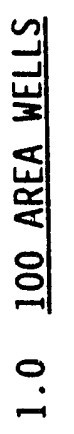

意䡈

'된

热管 000000000000000

夏

लI 000000000000000

总 000000000000000

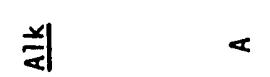

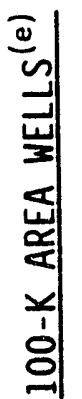

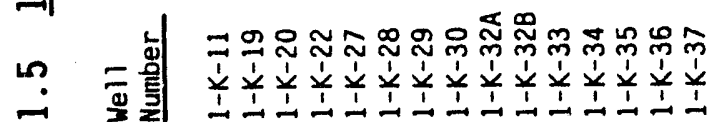

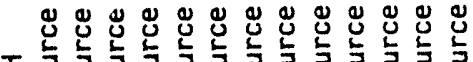

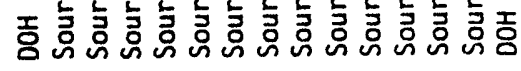

总 0 ह

学 0 है

矛部

I্

고퇴 일

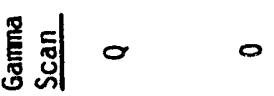

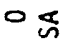

읭

离 $0000000<000000$ 嵌

홈회

'하

过算 $0000000<0$ 立

京

mI $0000000<000000$ 占

洁雨 $0000000<000000<$

$\frac{y}{4}<$

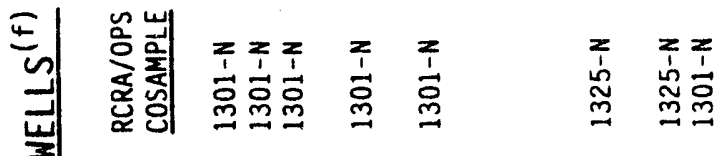

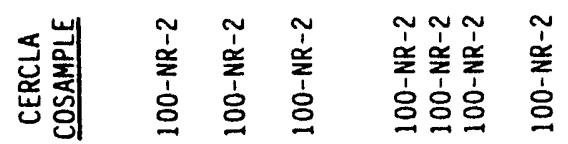

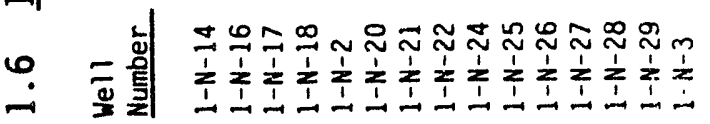




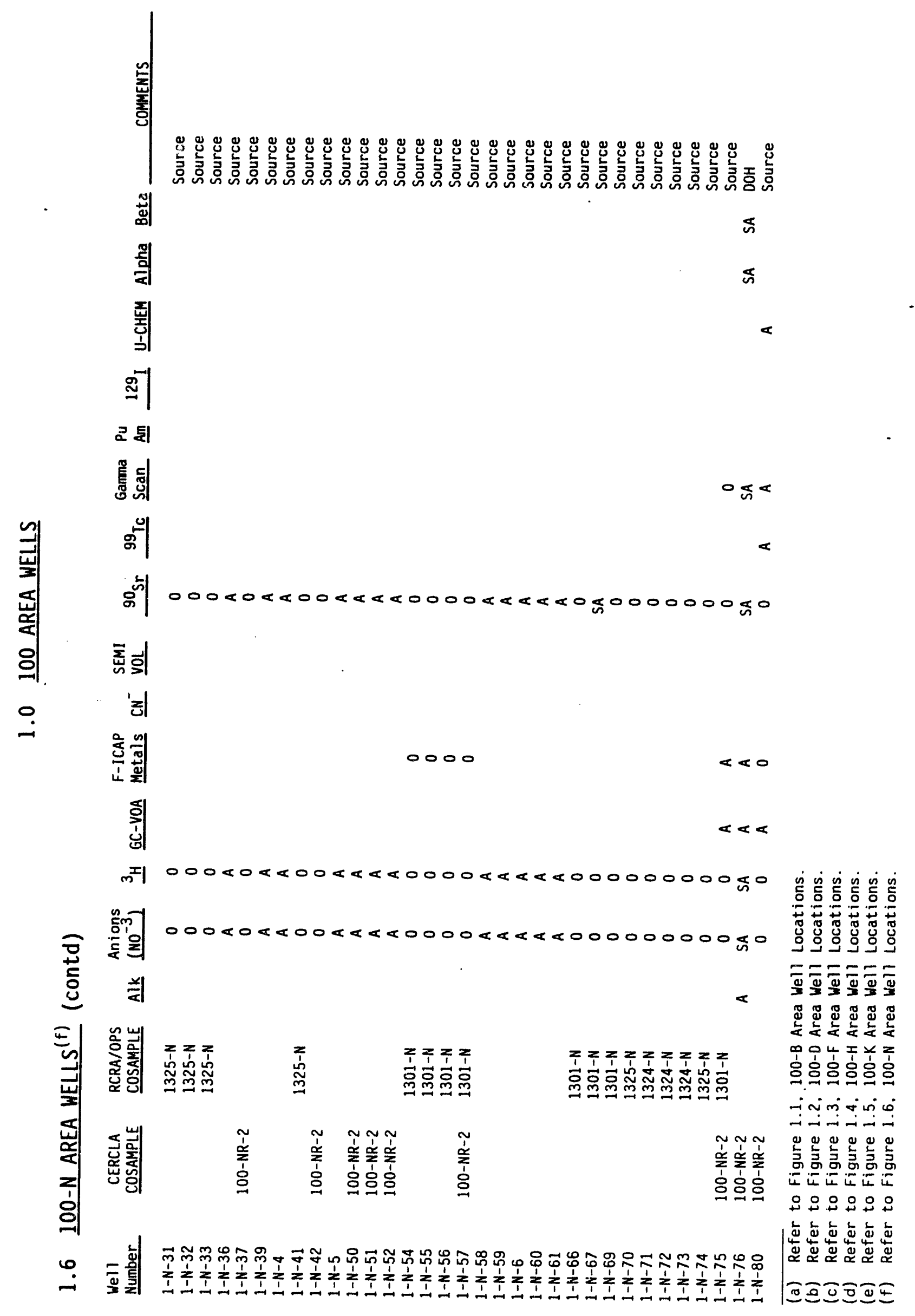




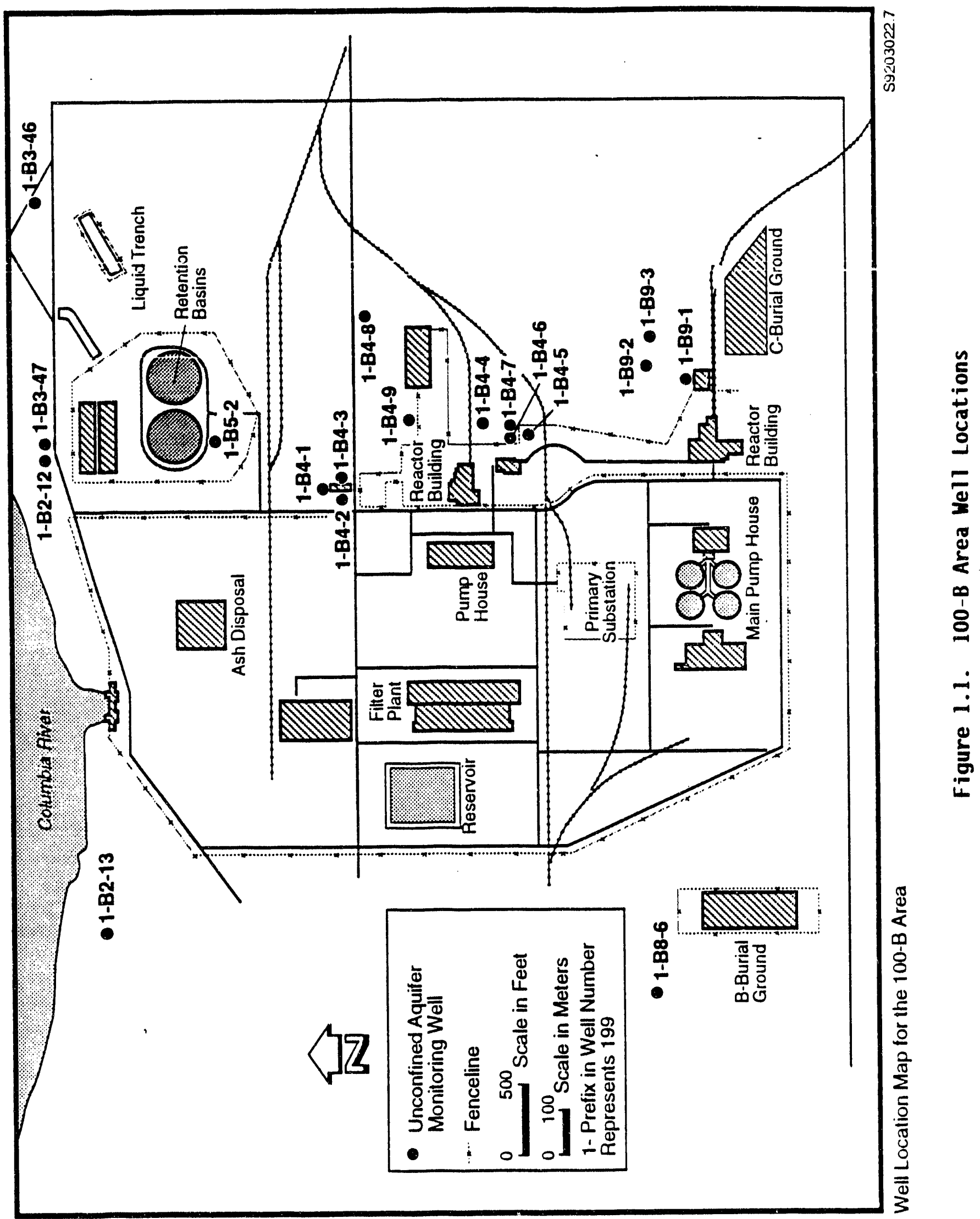




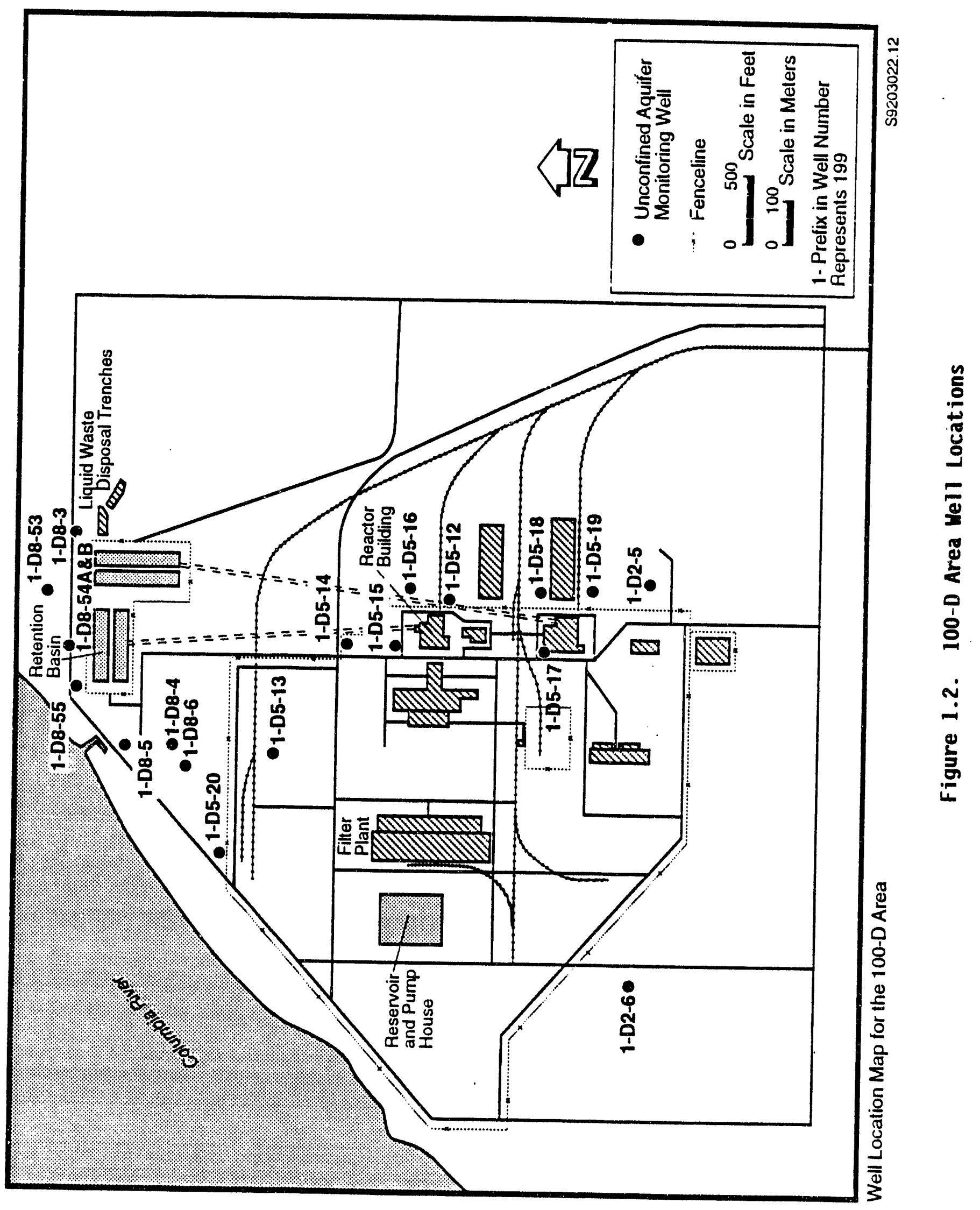




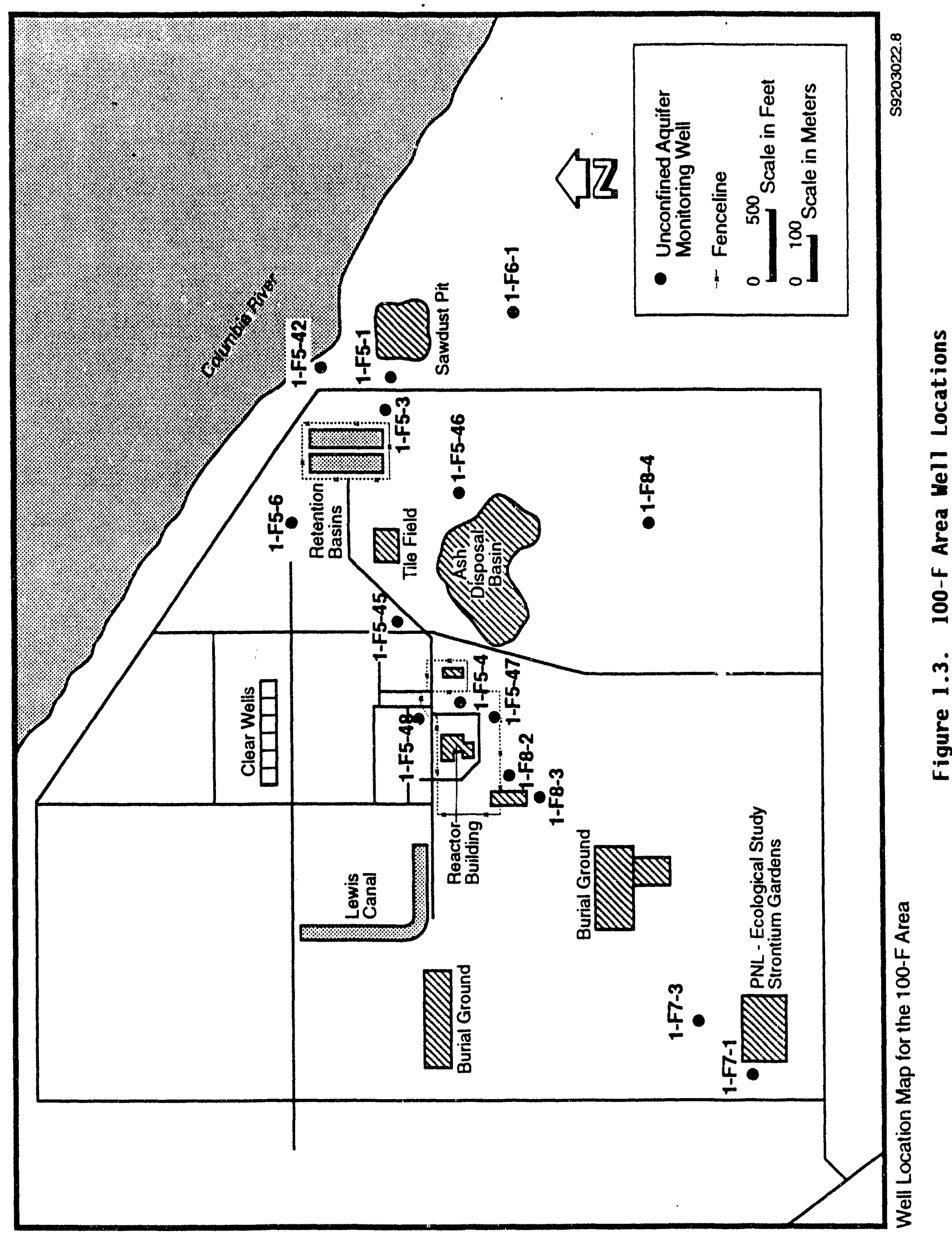




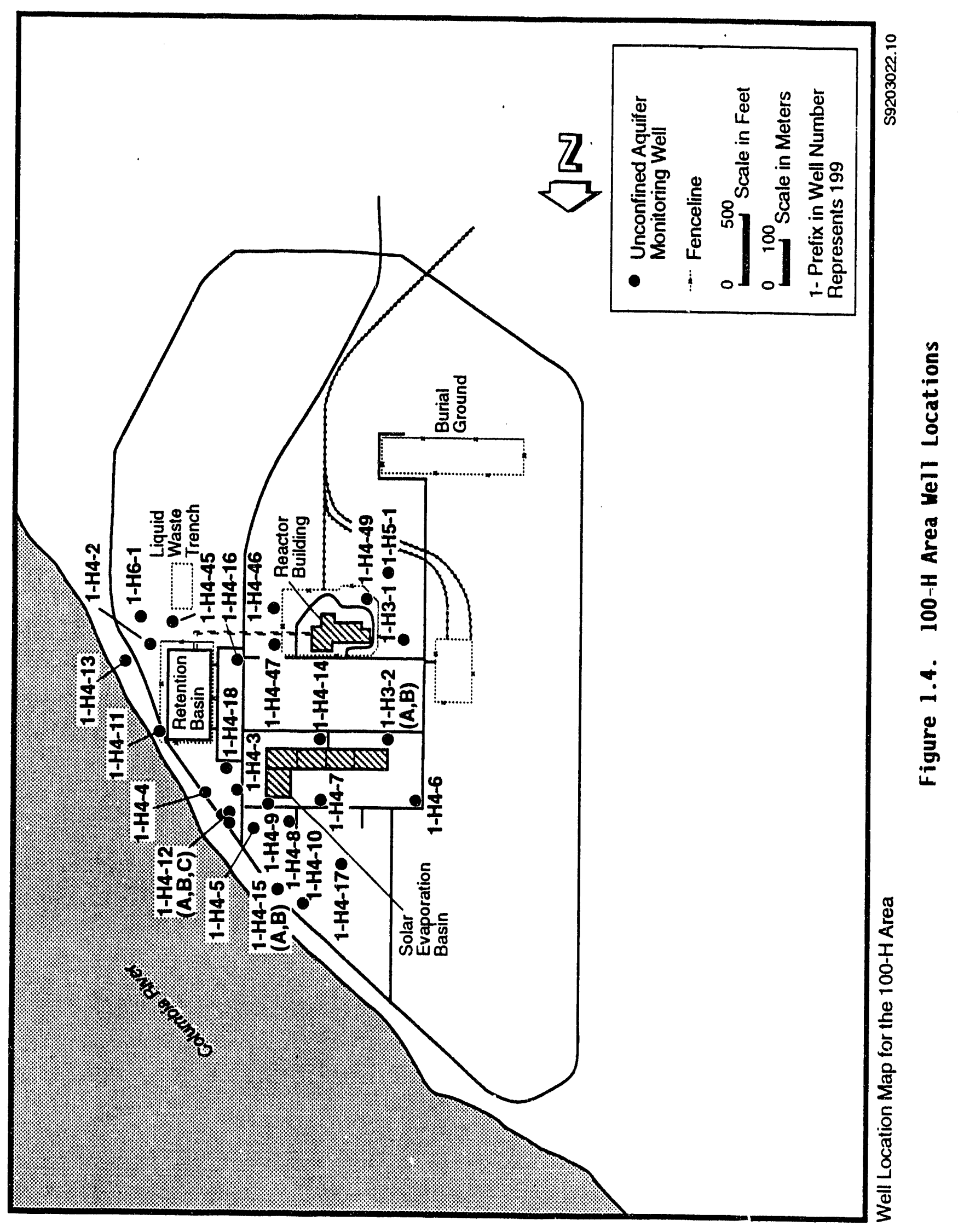




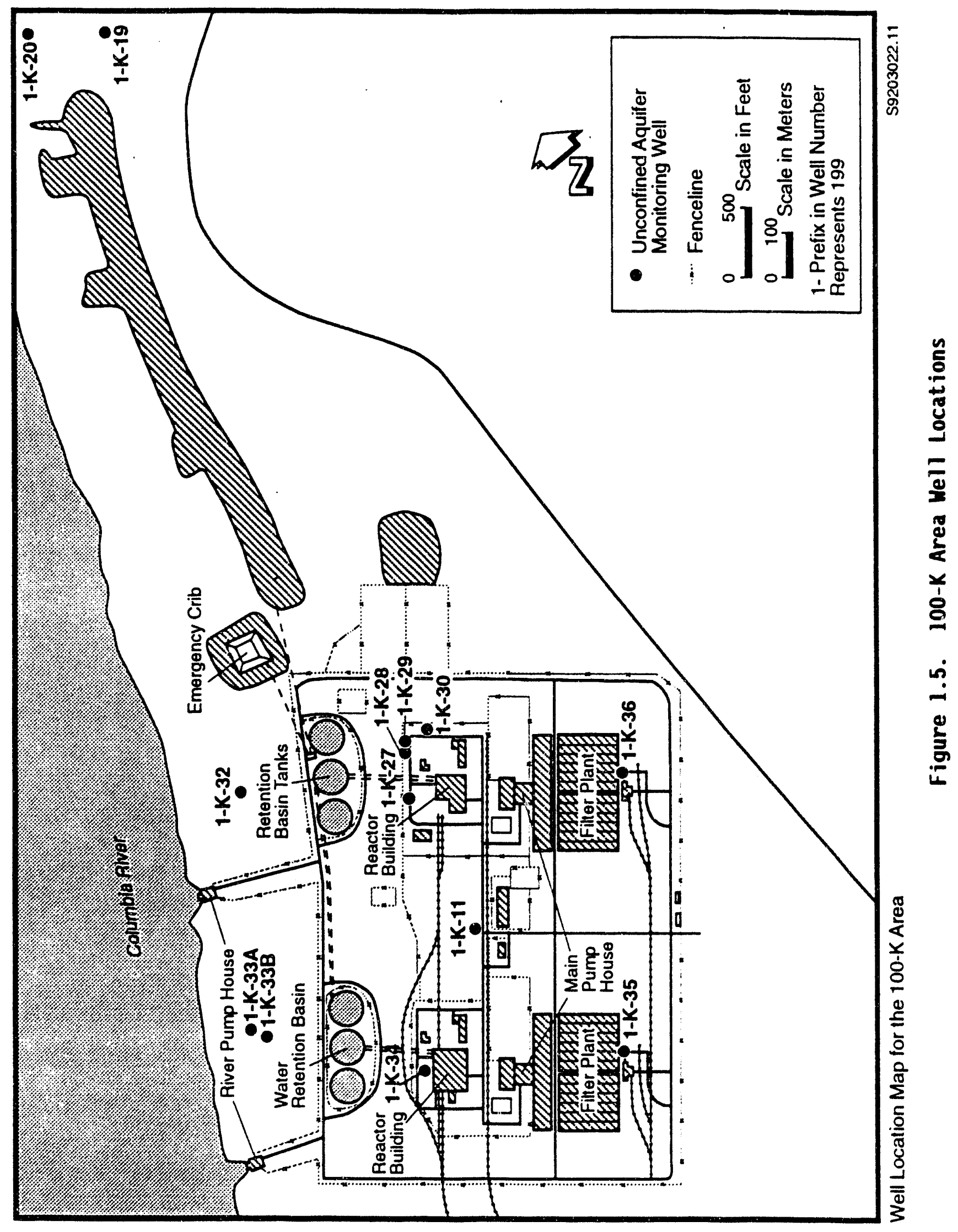




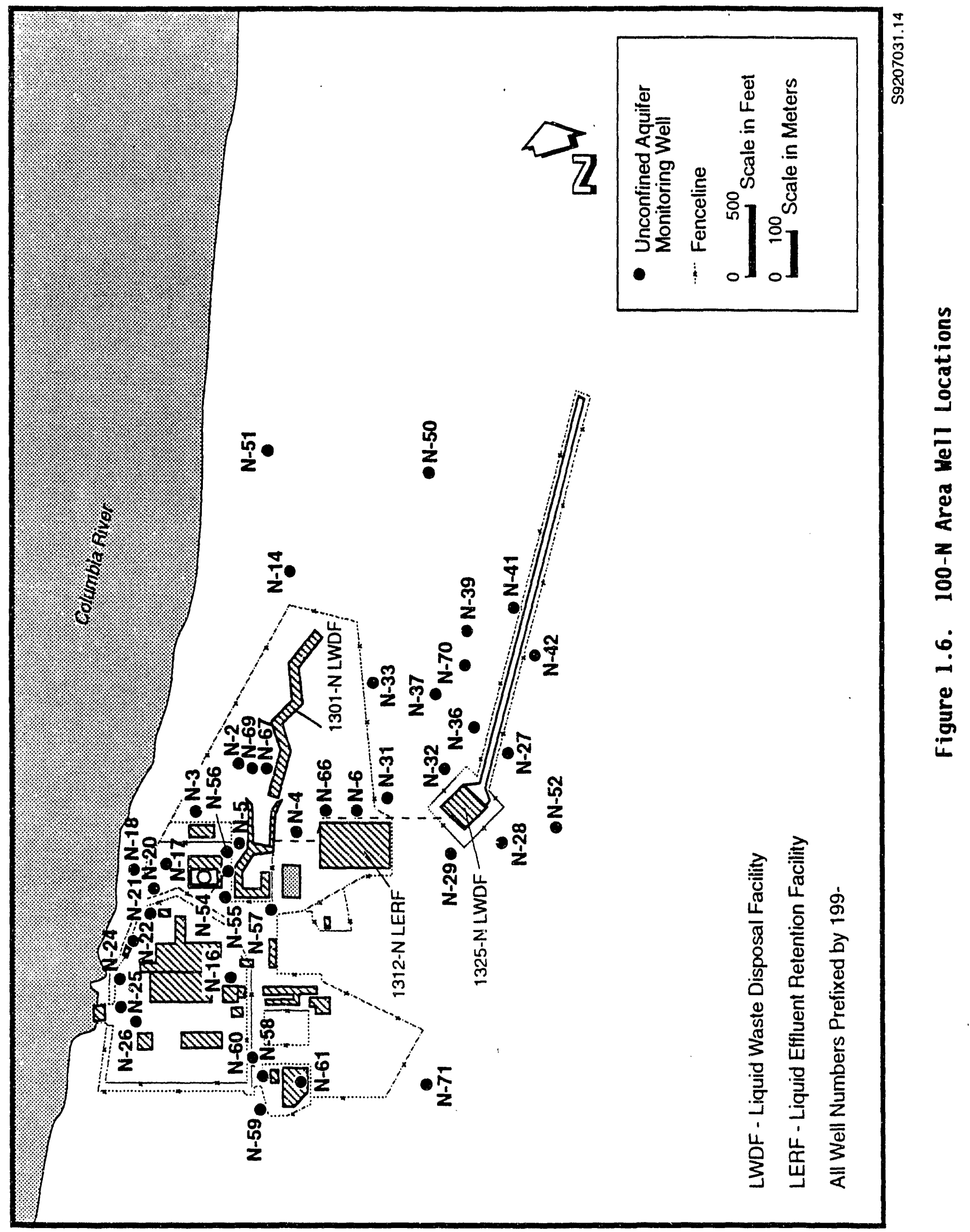


항

受 $\ll$

김

$<<0<<<<<<<0<0<<<<<<<<<-4<<<00000<<<<<<<$

조위

莺 哥

000000000

봎

g.

85 $14<0 \quad 000000<00<<0$

$<<<<<<<<<$

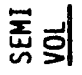

'단

$\stackrel{\circ}{i}$

空器

站

m| $<<000000000<00<<00000<<<<000000<<<0<<0$

웅ำ

$<<000000000<00<<00000<<<<000000<<<0<<0$

娄

늠현

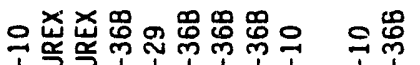

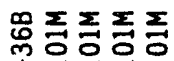

䒺

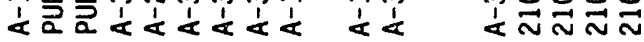

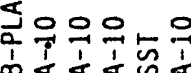

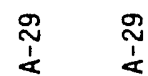

矛耐

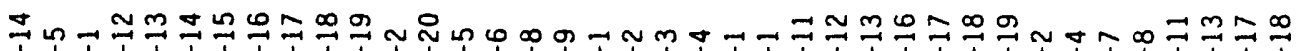

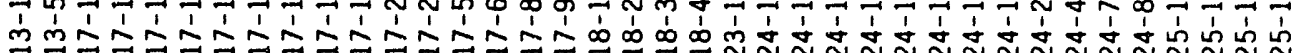

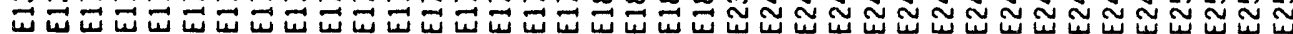

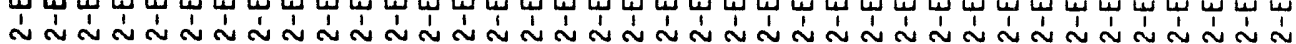


ํํำ

章

害

惯

$m^{I} \mid 0000<0<<<<0<0<<00000000<<000000000<$

$0000<0<0<00000000000000<<000000000<$

前

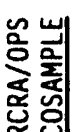

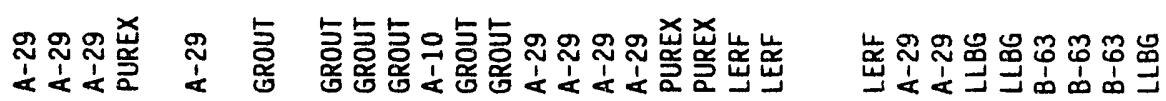

运岂

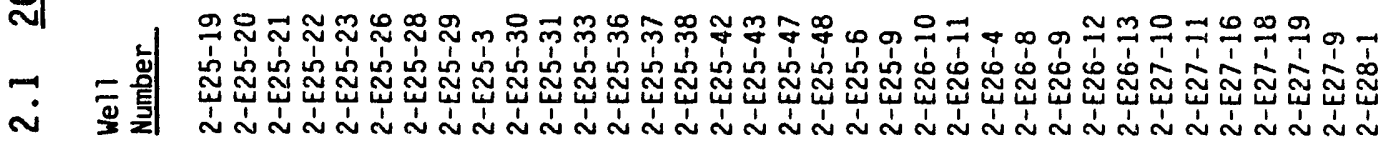




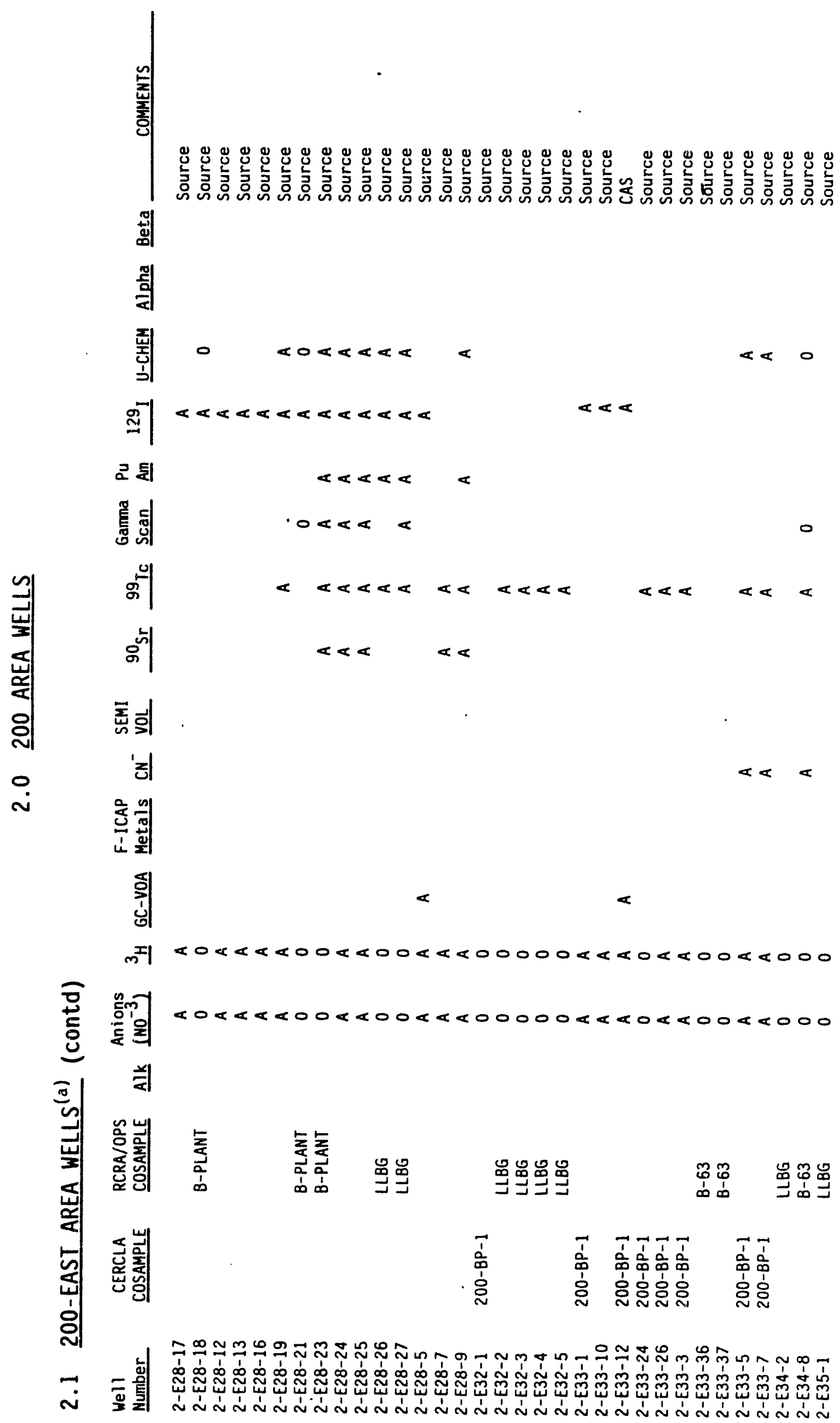




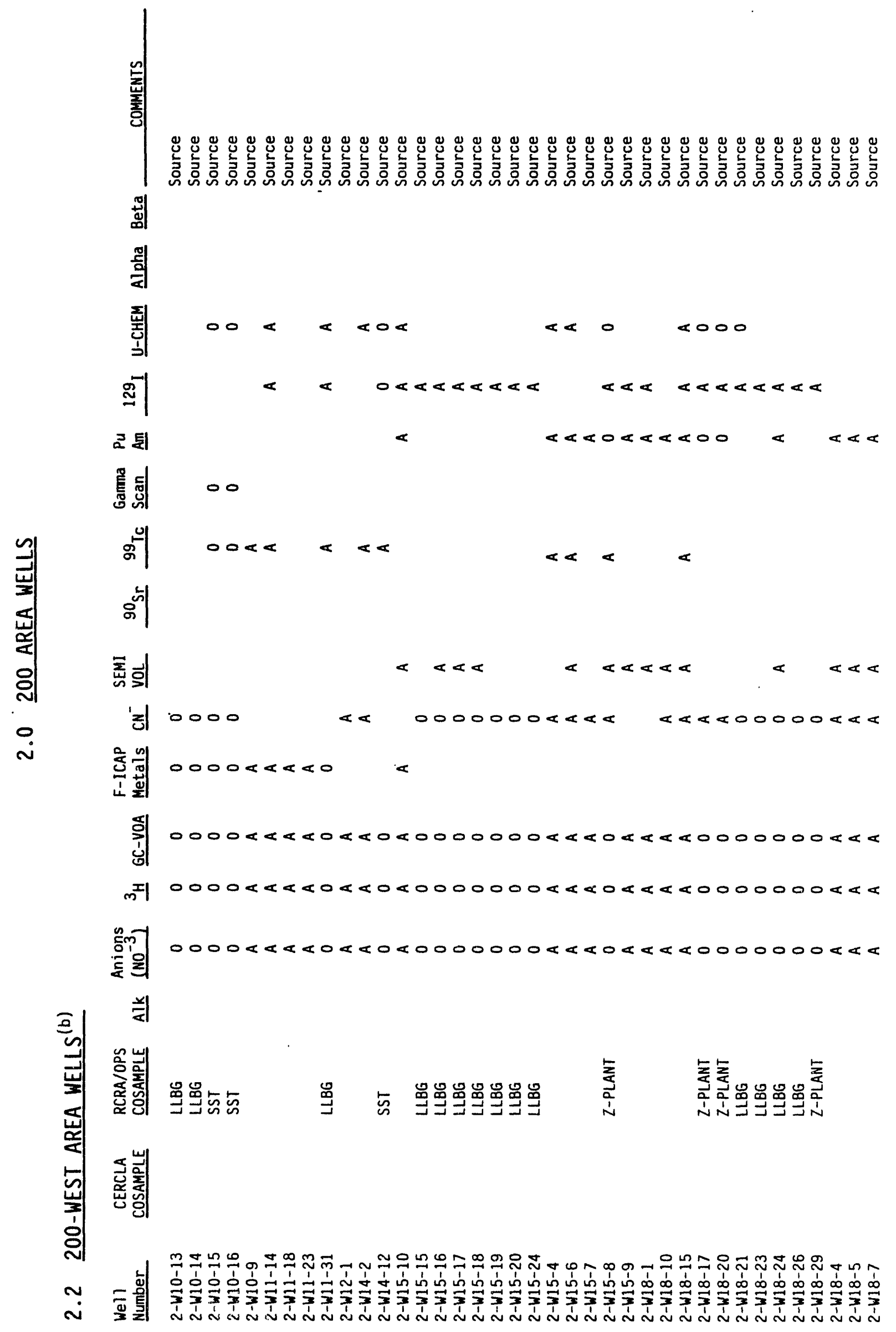




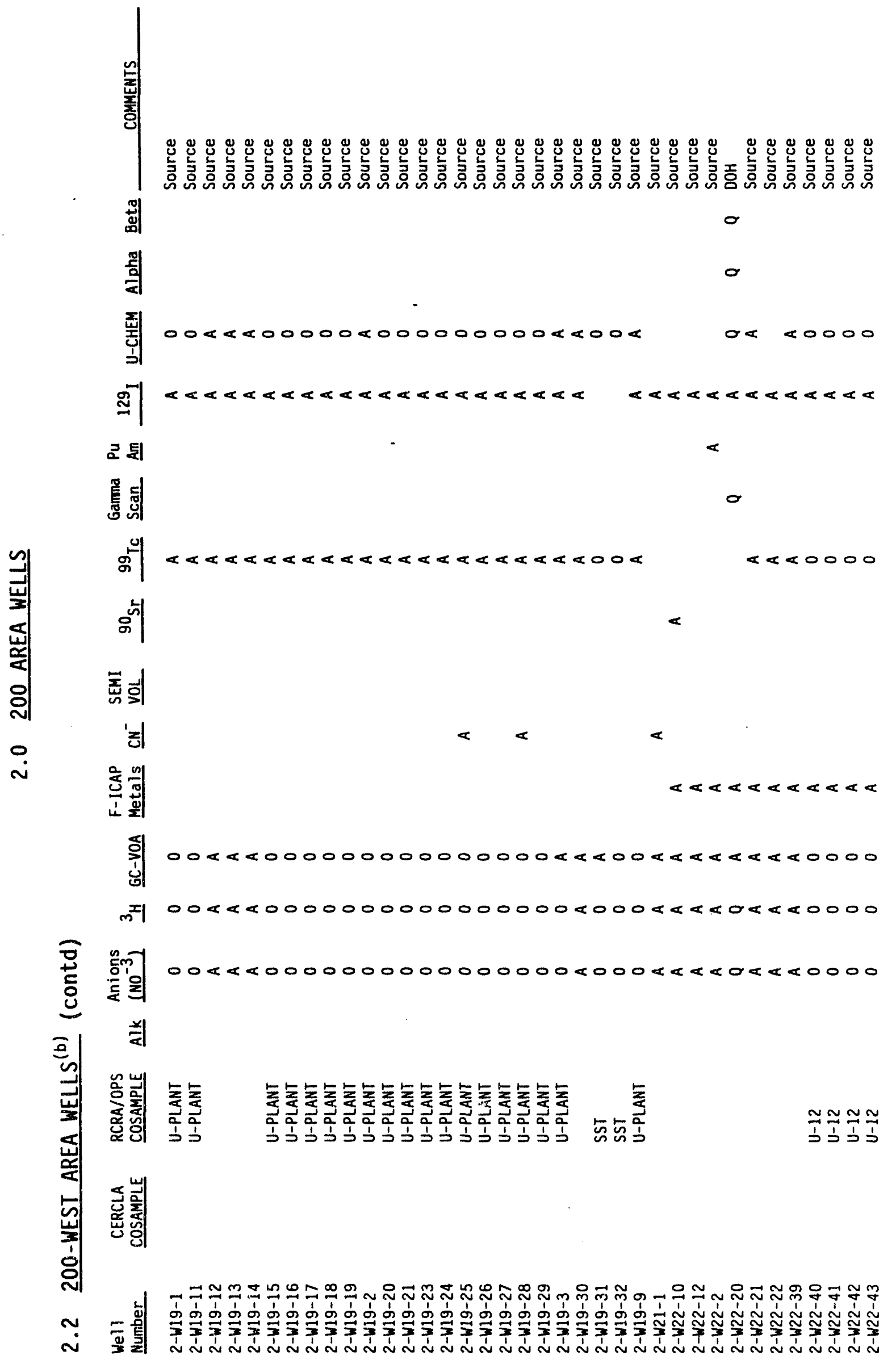




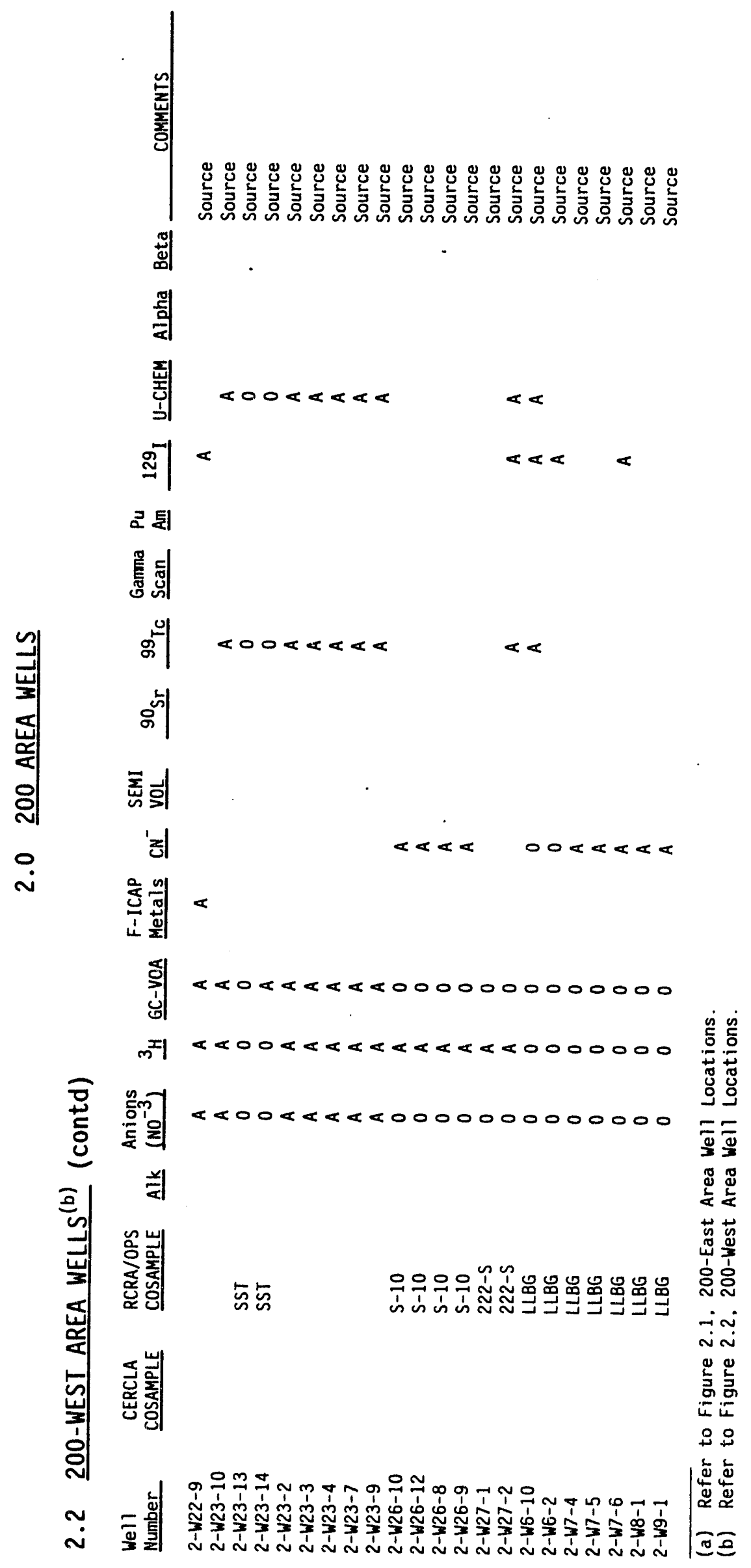




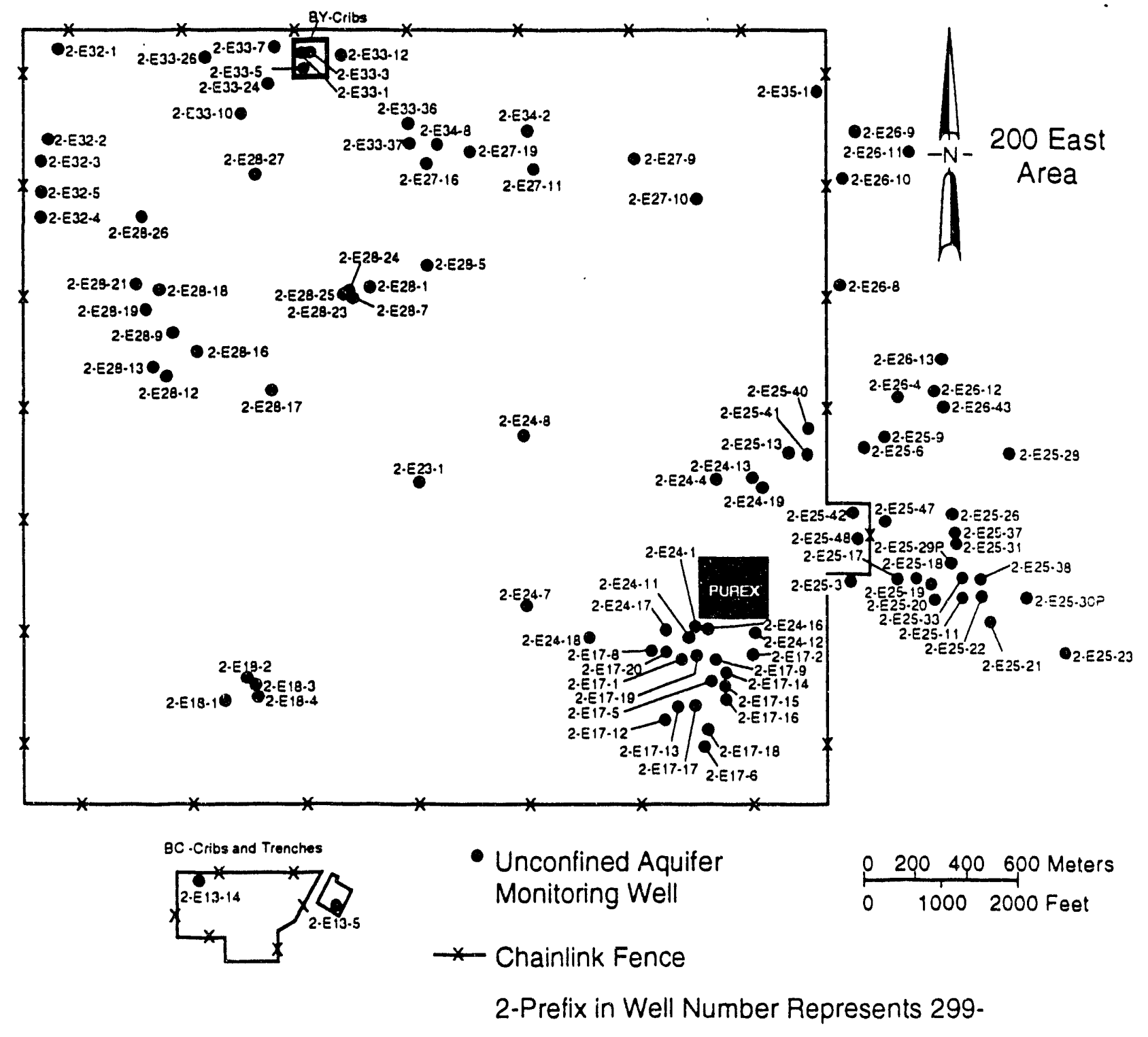

Figure 2.1. 200-East Area Well Locations 


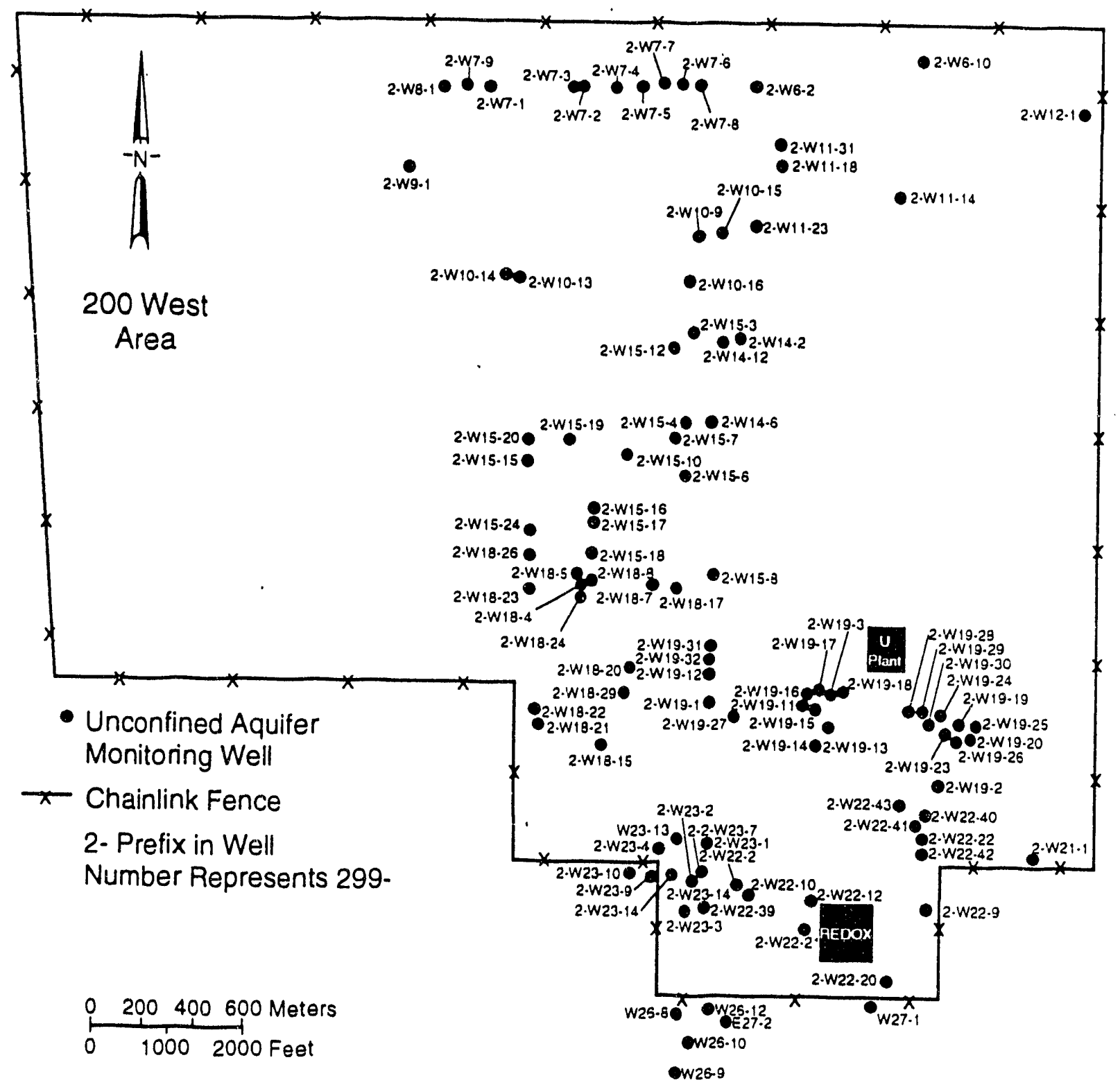

$\$ 9212057.2$

Figure 2.2. 200-West Area Well Locations 


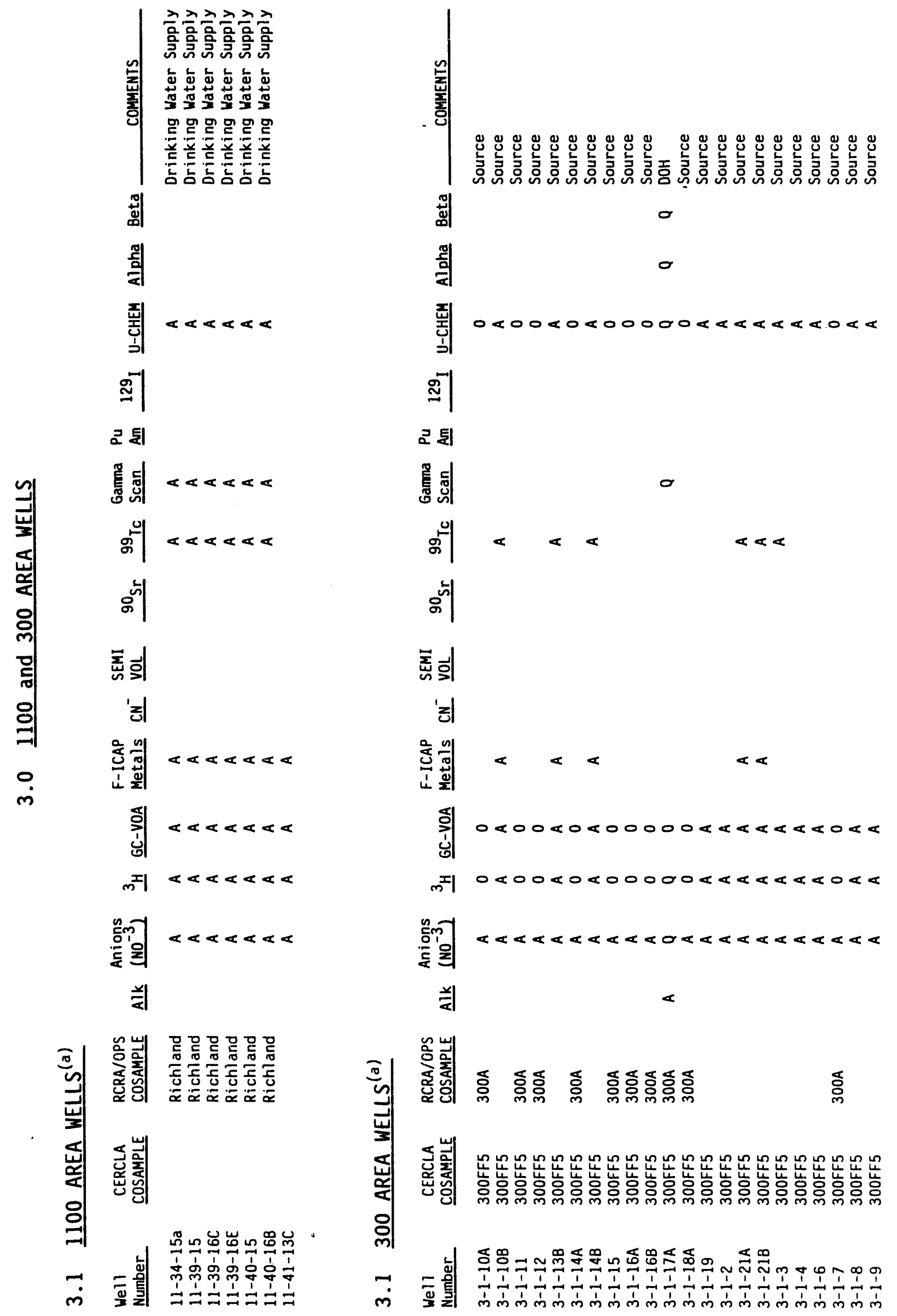




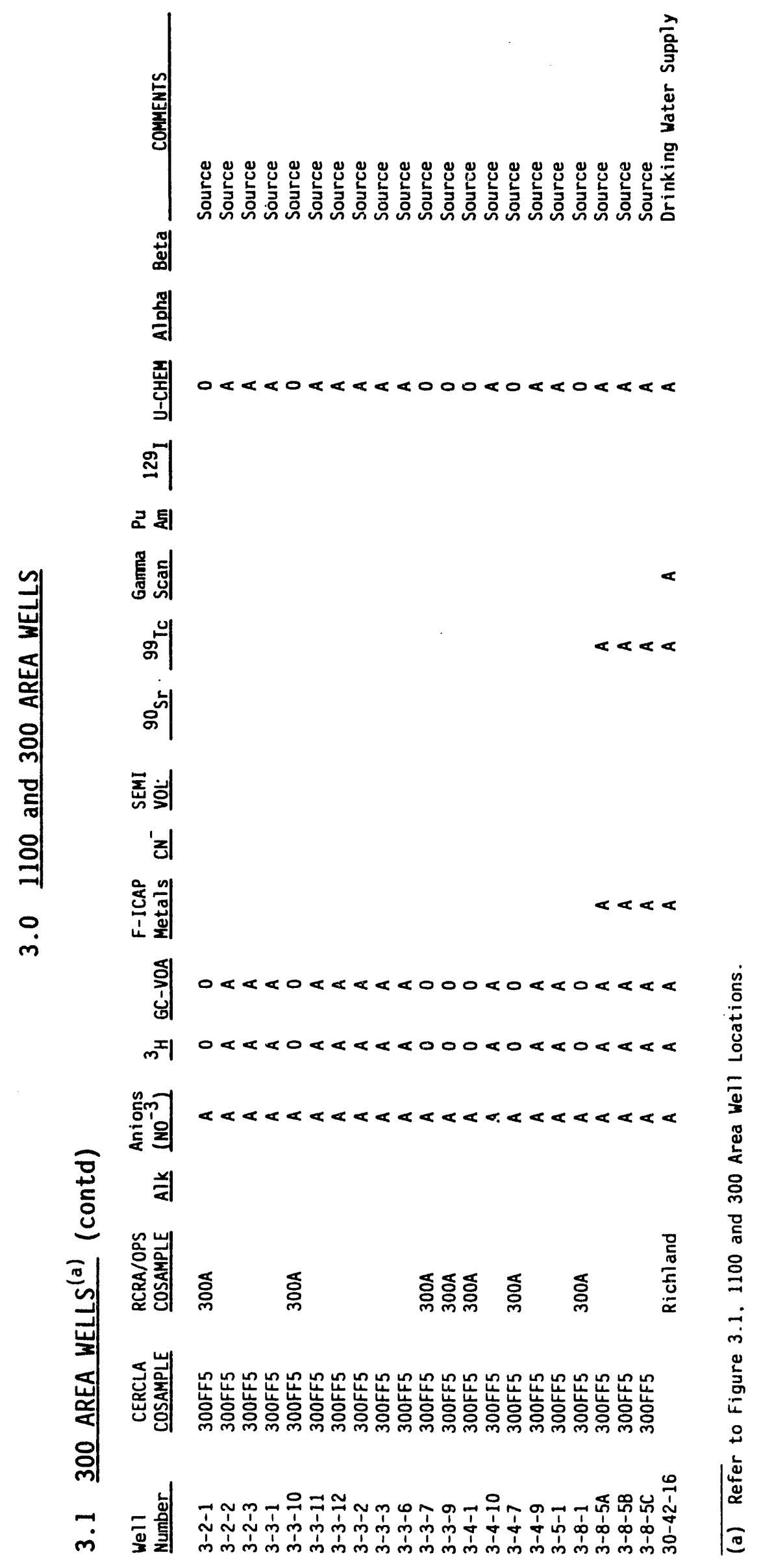




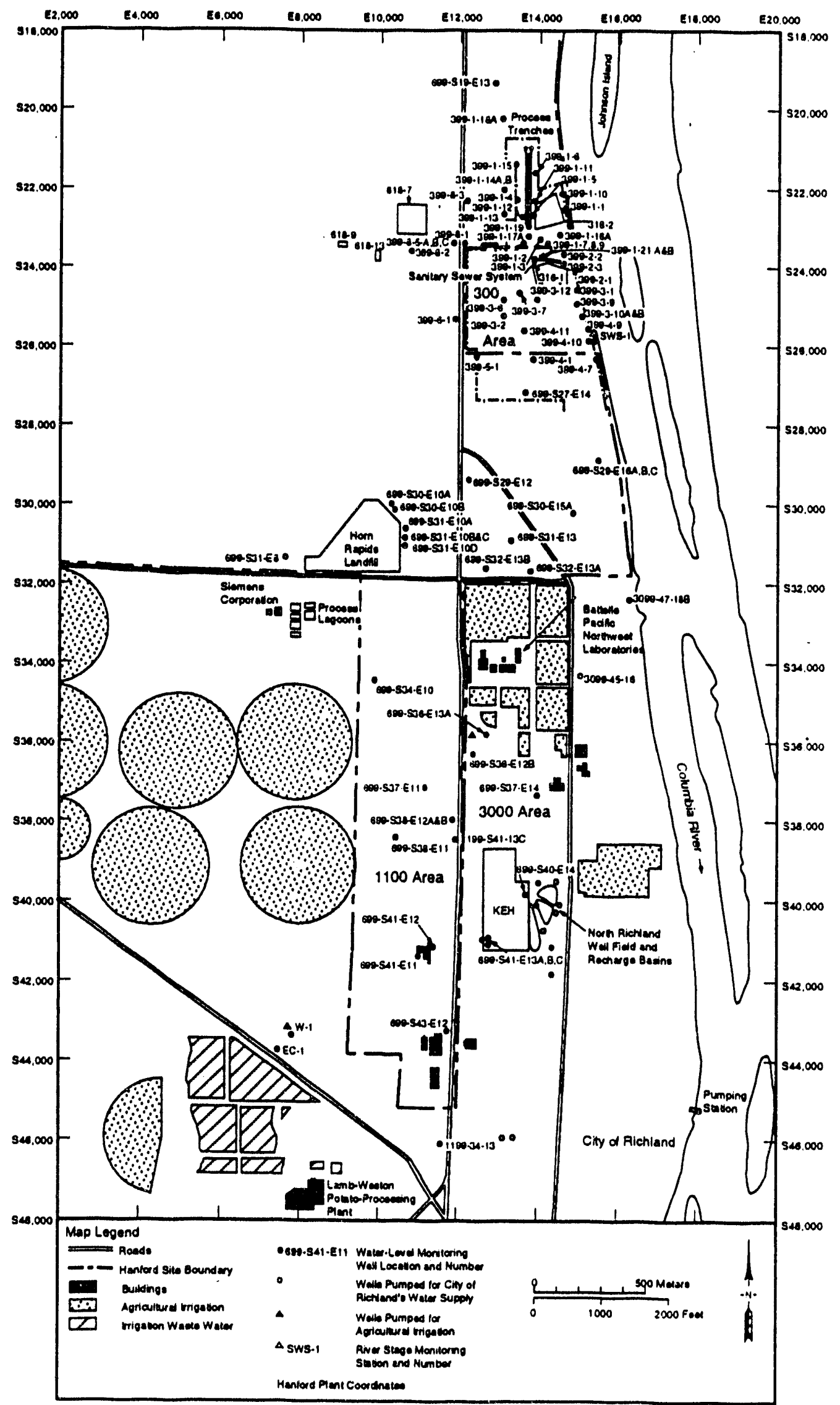

58212057.

Figure 3.1. 1100 and 300 Area Well Locations 


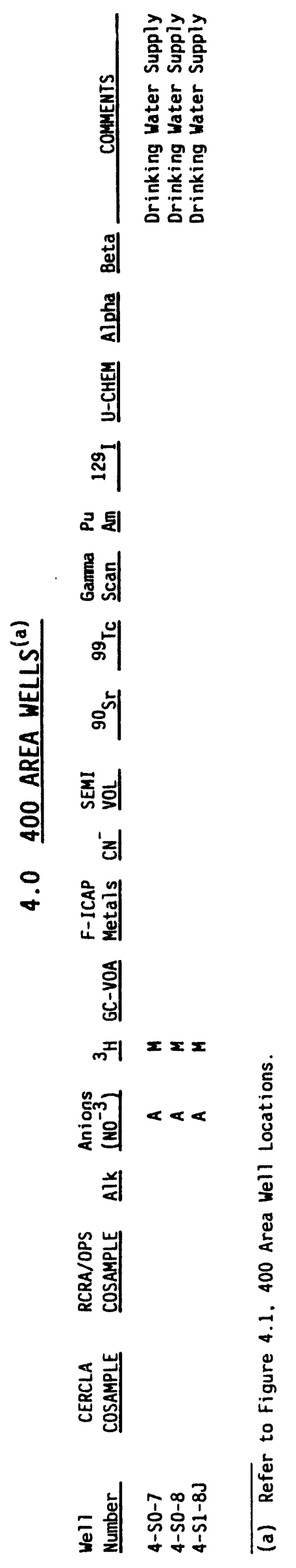




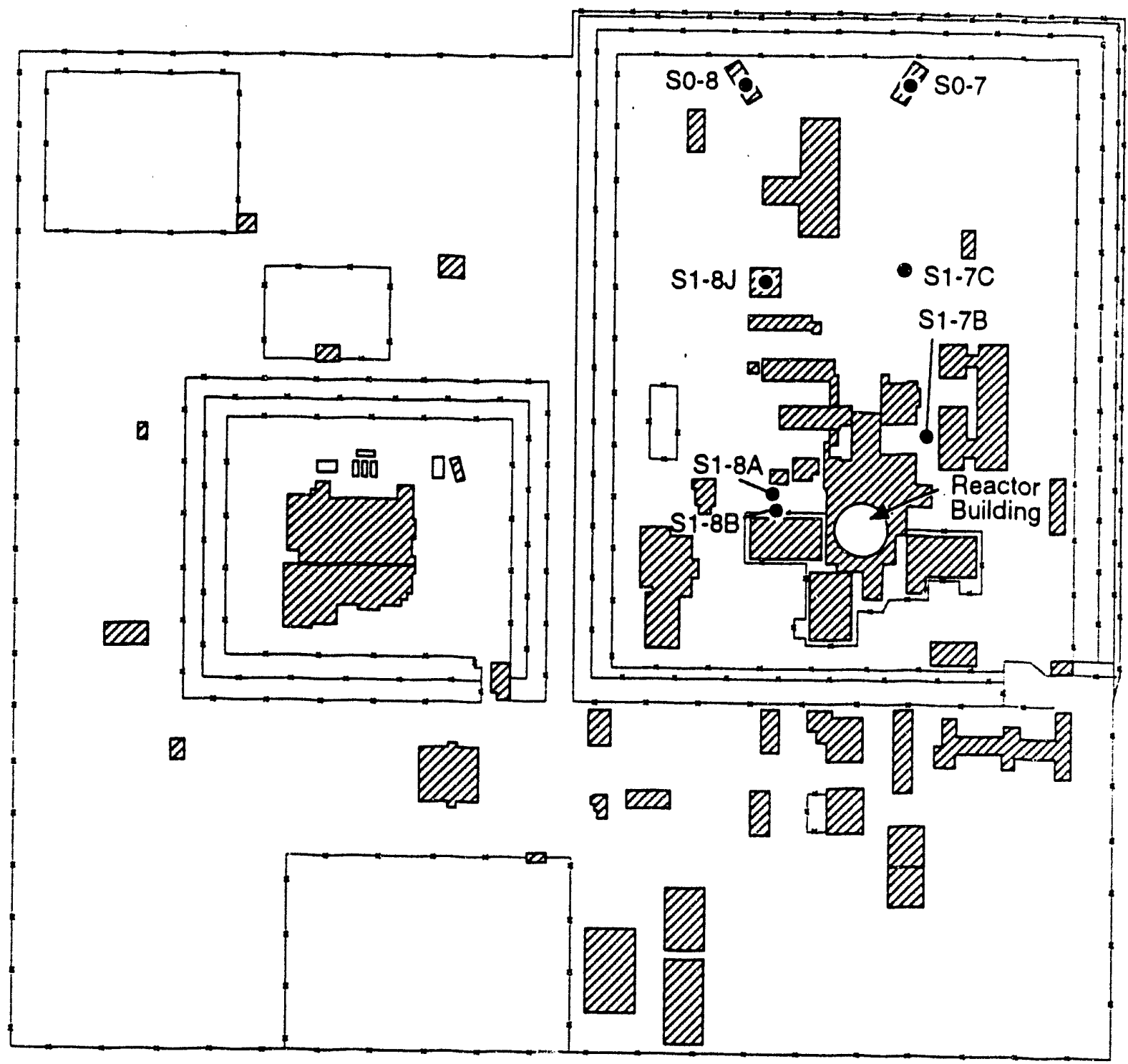

All Well Numbers Prefixed by 499

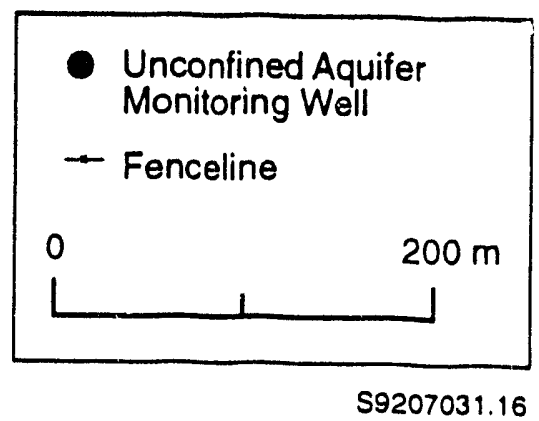

Figure 4.1. 400 Area Well Locations 
\& 0

\& $<\quad$

$<\sigma$

矛

$\stackrel{9}{\Xi}$

$\leftarrow$

공

茞 矛

\&

o

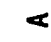

$<$

$<$

$<$

ฒ

ज्ञ

密잉

인 횐

요

$\ll<\ll<$

$<<<<<<<$

离

$m^{x}$ $4<<<1<$

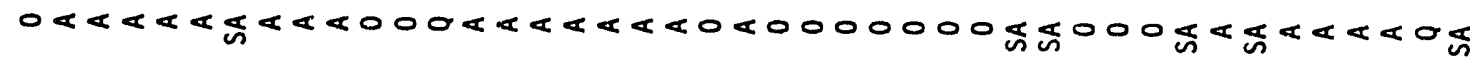

농ํำ

$0<<<<<\&<<<000<<<<<<<0<0000000<<000<<<<<<<0<$

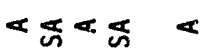

号就

$\S$

审守

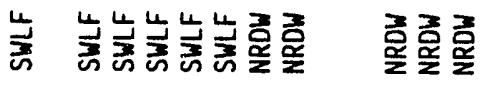

这㒬

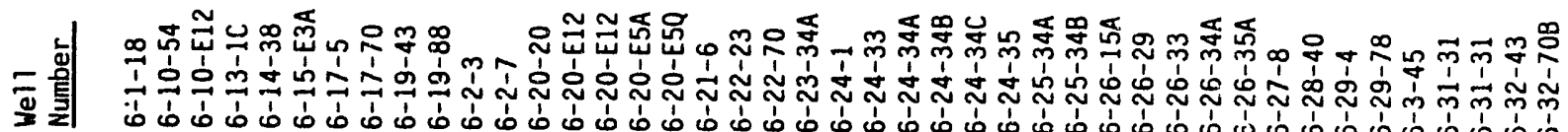




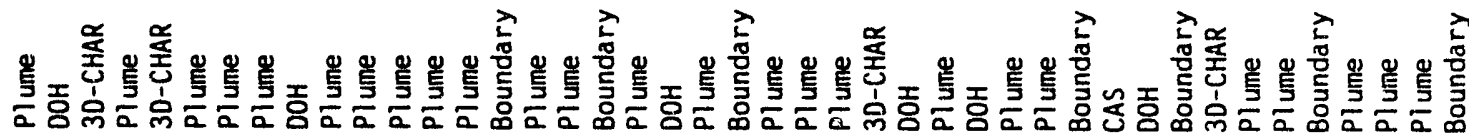

웨 $<$

웡

풀

ำ

룽

兽 哥

苍苛

웅

폈의

춘

م.

ら

희

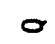

$\circ$

$0 \leftleftarrows$

$<<$

$\circ$

$<<$

这势

의

ปั่

$m^{I}$

둥의

当

농현

志兘

¿

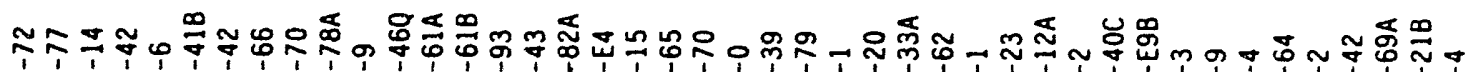

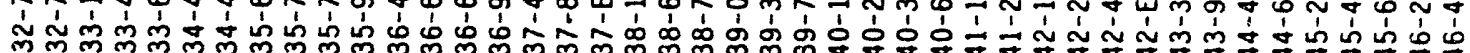

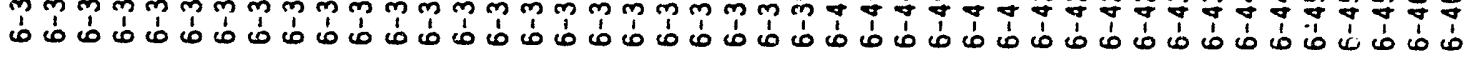




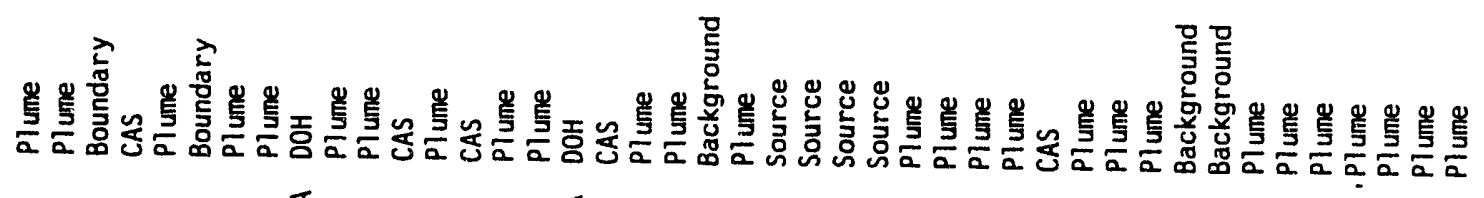
ज s

휭 \&

矛

$\stackrel{2}{2}$ s $\pi$

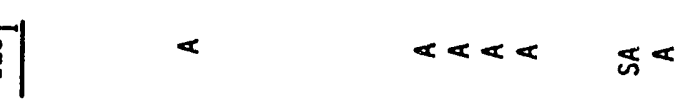

르ㄴㅚㅟ

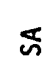

哭 듕

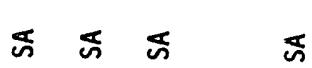

$\leftarrow<$

战

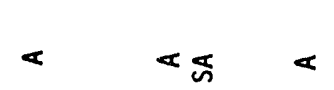

密 이

인

م) 兘

$\frac{0}{0} \frac{0}{0} \quad \ll<<<$

\&

$\ll \ll$

$\propto<\ll$

$\varangle \quad<\ll$

离|
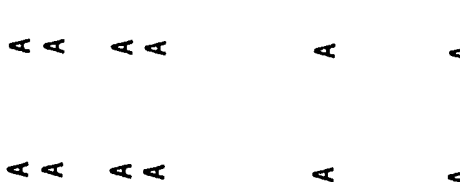

$<$

$<$

$\ll<\ll$

$n^{\text {II }}$

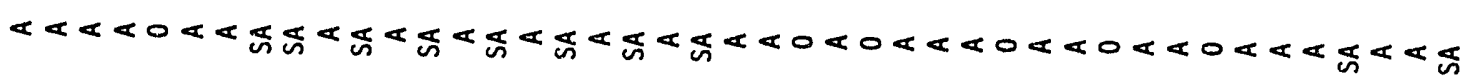

등ำ

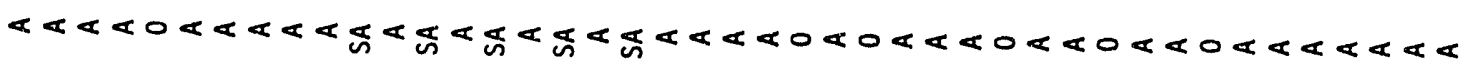
$\frac{\ddot{a}}{\mathrm{a}}<<<<$

号严

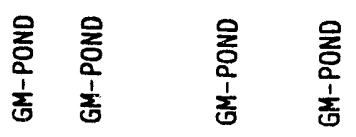

选高

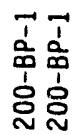

$\begin{array}{ll}1 & 1 \\ 1 & 0 \\ 0 & 1 \\ 1 & 1 \\ 0 & 0 \\ N & 0\end{array}$

$\begin{array}{ll}i & 1 \\ \vdots & \vdots \\ 0 & 0 \\ 1 & 1 \\ 0 & 0 \\ 0 & 0\end{array}$

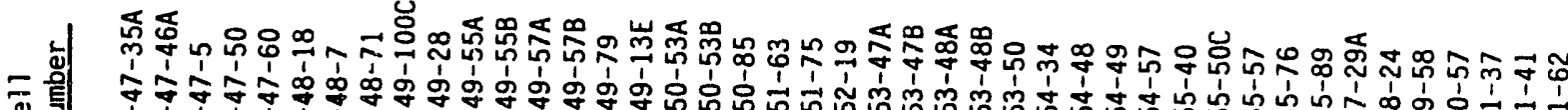

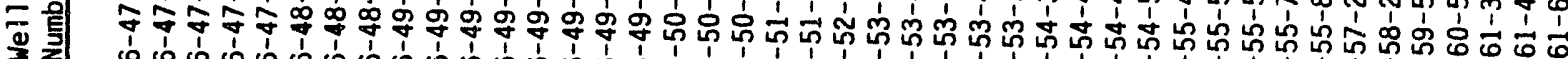
○ 


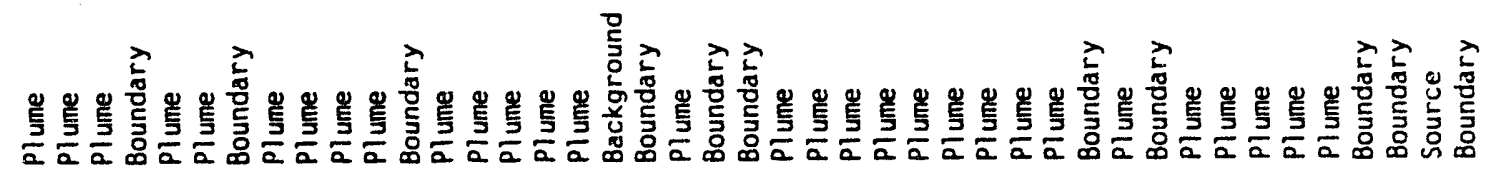

- ث્|

윙

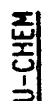

피

루 튕

篦

$<$

$<\alpha<\quad<$

焉

品

이 형

in

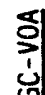

m

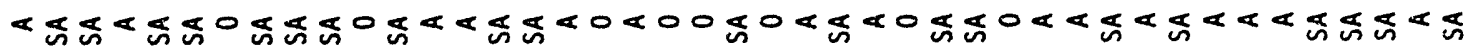

둘욜

$<<<<<<0<<<0<<<<<<0<00<0<<<0<<0<<<<<<<<<<<<<$

当

享념

놀면

$\begin{array}{ll}0 & 0 \\ \dot{y} & 0 \\ 0 & 0 \\ 0 & 0 \\ 0 & 0\end{array}$

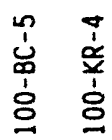

京

竞总紊

غㅇㅇㅇㅇㅇ

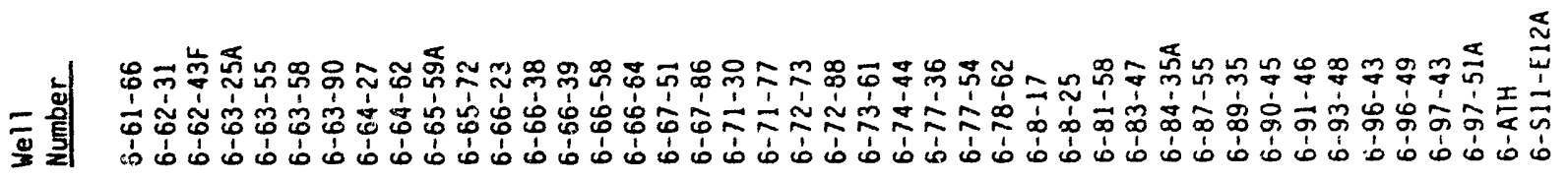




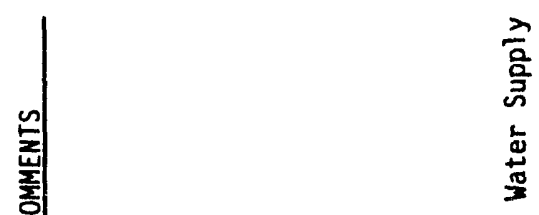

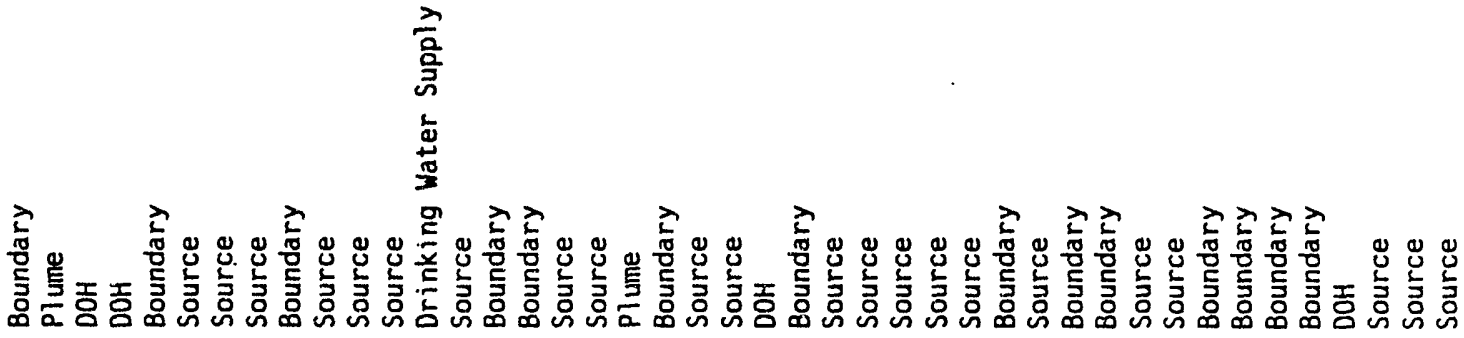

敬

00

哭

焉

$\infty 0$

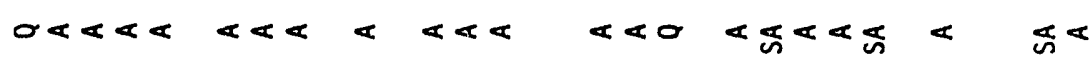

ฐ్స

롱

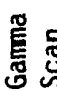

-

$\circ$

o

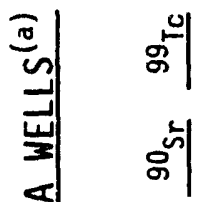

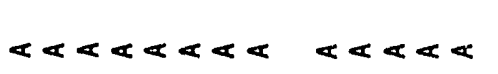

$<<<$

$4<<<<<<$

$\ll<$

o

密

잉

친

is

悹悹新

$<<<<<<<<$

$\ll<<<<<$

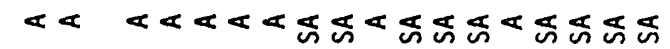

๒์ธ์

站

|

$m^{I}$

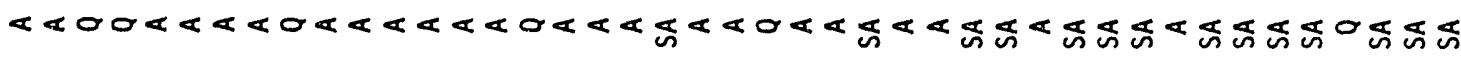

둥ํำ

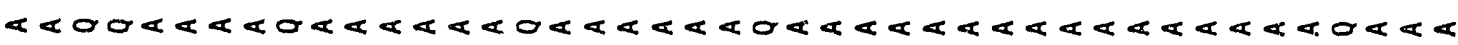

羊

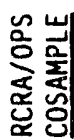

岕趗

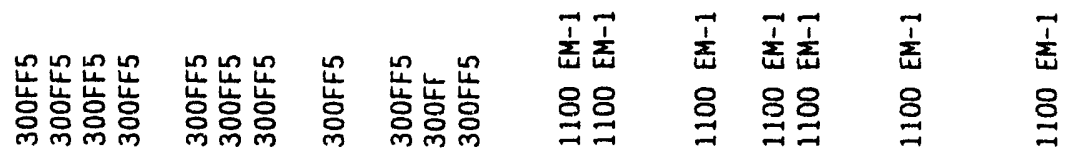

范

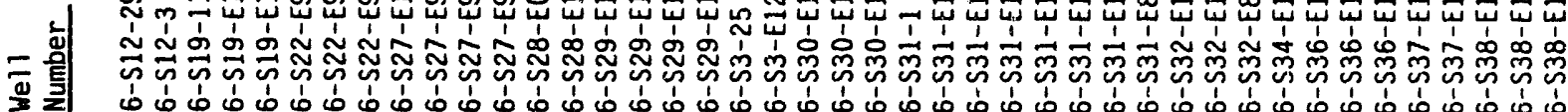

बू 


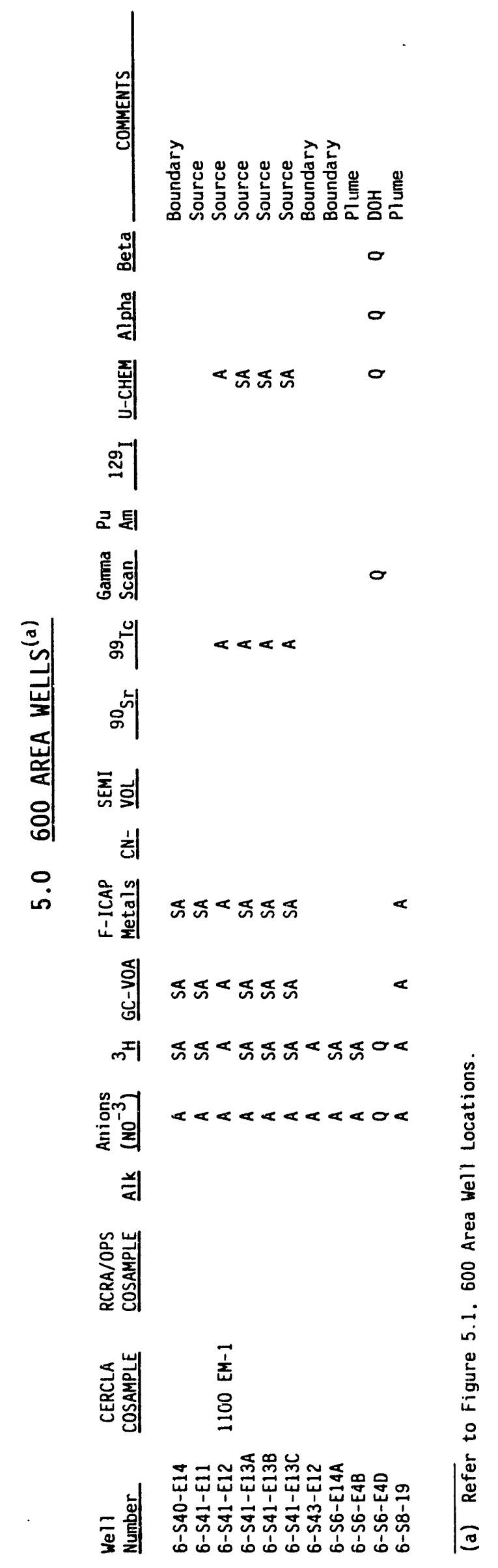




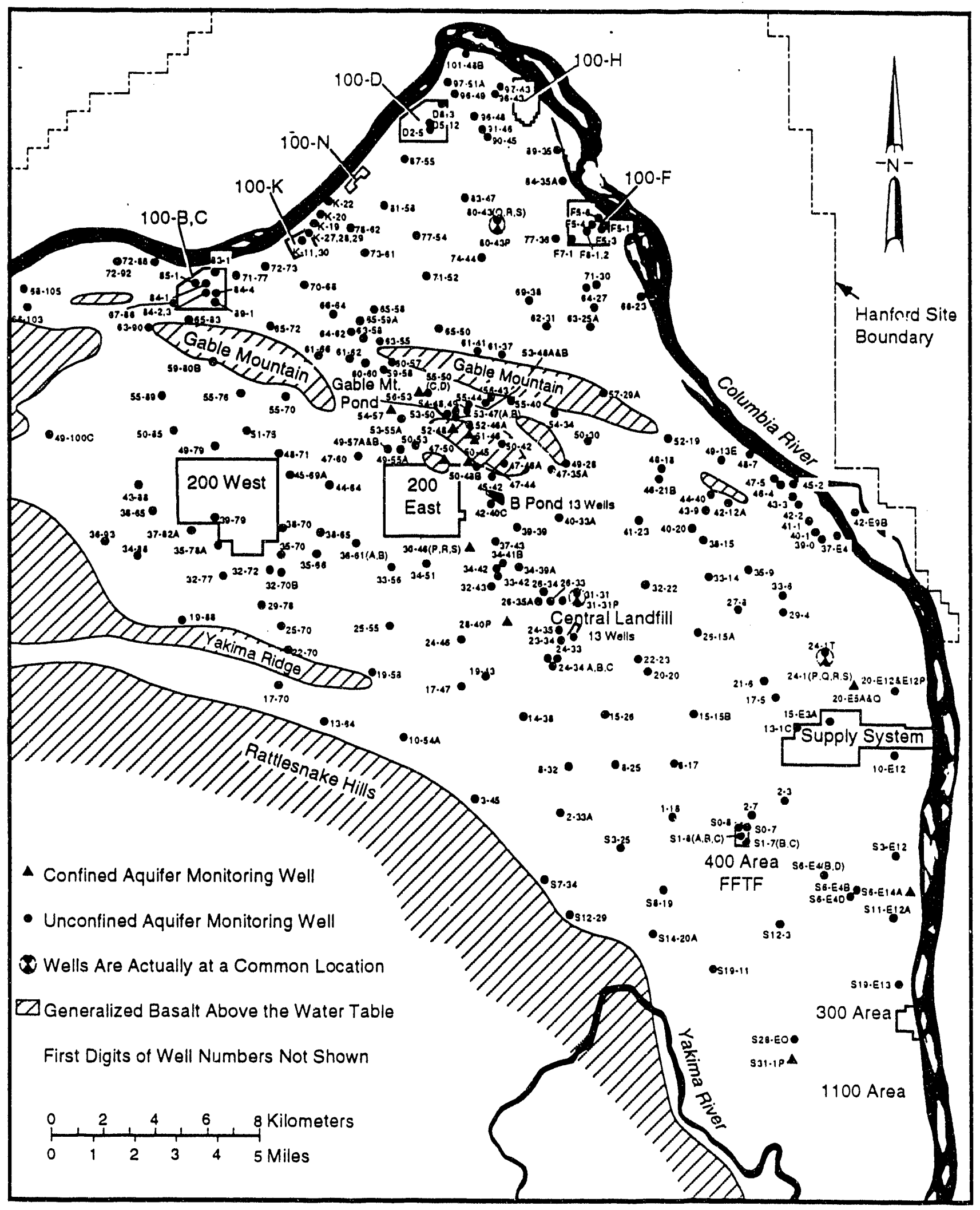

s9212057.9

Figure 5.1. 600 Area Well Locations 
No. of

Copies

\section{OFFSITE}

12 DOE/Office of Scientific and Technical Information

C. E. Cline

Washington State Department of Ecology

99 South Sound Center

MS 7600

Olympia, WA 98504-7600

P. T. Day

U.S. Environmental Protection Agency

P.0. Box 550

MS B5-01

Richland, WA 99352

J. L. Erickson

Division of Radiation Protection Radiation Control Section

Washington State Department of Health

Airindustrial Center, B1dg. 5

MS LE-13

Olympia, WA 98504

Dave Jansen

Washington State Department of Ecology

99 South Sound Center

MS 7600

OTympia, WA 98504-7600

K. Kowalic

Washington State Department of Ecology

99 South Sound Center

MS 7600

Olympia, WA 98504-7600

3 Dave Nilander

Washington State Department of Ecology

7601 West Clearwater Street

Suite 102

Kennewick, WA 99336
No. of

Copies

R. D. Paris

Radiation Control Section

Oregon State Health Division

1400 SW 5th Avenue

Portland, OR 97201

2 D. R. Sherwood

U.S. Environmental Protection

Agency

P.0. Box 550

MS B5-01

Richland, WA 99352

6 Community-Operaced Environmental

Station Manager's

K. A. Darrington

3024 \#A W. Opal

Pasco, WA 99301

E. Corralus

1540 Filbert Rd.

Othello, WA 99344

T. E. Gilmore

2120 Hamilton

Richland, WA 99352

M. P. Madison

1715 N. 18th Avenue

Pasco, WA 99301

C. L. Stevenson

70 Canal Drive

Mesa, WA 99343

C. A. Wagner

1528 Hunt Avenue

Richland, WA 99352

\section{ONSITE}

17 DOE Richland Field Office

R. F. Brich A5-55

M. J. Furman A5-21

E. D. Goller A5-19

J. D. Goodenough A5-19 
Copies
A. C. Harris A5-19
R. D. Hildebrand A5-55
R. G. Holt A5-15
R. A. Holten A5-55
R. G. Mcleod A5-19
P. M. Pak A5-19
R. K. Stewart A5-19
K. M. Thompson A5-15
M. W. Tiernan (5) A5-55

\section{Hanford Environmental}
1 Health Foundation

P. A. Thurman B1-61

1 U.S. Corps of Engineers

W. L. Greenwald A5-20

24 Westinghouse Hanford Company

M. R. Adams H4- $=5$

G. D. Carpenter H6-20

L. B. Collard H4-14

L. P. Diediker T1-30

J. J. Dorian H6-20

K. R. Fecht H6-06

K. A. Gano X0-21

E. M. Greager L6-60

R. L. Jackson H6-06

V. G. Johnson H6-06

A. J. Knepp H6-06

M. J. Lauterbach H4-55

A. G. Law H6-06

S. M. McKinney T1-30

R. M. Mitchell H6-04

D. L. Parker H6-03

R. E. Peterson H6-06

J. W. Roberts H6-02

S. E. Rowe K4-13

J. A. Serkowski H6-06

L. C. Swanson H6-06

S. J. Trent H6-06

S. E. Vukelich H6-06

C. D. Wittreich H4-55

91 Pacific Northwest Laboratory

M. E. Almarode K6-78

G. L. Andersen K6-78

E. J. Antonio K6-13

D. J. Bates K7-34
Copies

L. L. Belt K6-78

L. E. Bisping (30) K6-13

R. W. Bryce (10) K6-96

A. T. Cooper K6-13

R. L. Dirkes K6-13

S. P. Downey K6-81

P. E. Dresel K6-81

A. W. Endres P7-01

J. C. Evans K6-81

K. L. Farmer K6-78

J. J. Fix P7-01

S. L. Friant K6-13

R. W. Hanf, Jr. K6-13

L. W. Hankel K6-78

J. D. Harrison K6-78

P. S. Henry K6-78

E. L. Hilty K6-96

J. A. Jahnke K6-78

R. E. Jaquish Kl-30

J. L. Julya K6-78

G. V. Last K6-96

J. J. Lopez K6-78

E. W. Lusty K6-78

D. L. Mackliet K6-78

D. A. Mueller K6-78

K. B. 01 sen K6-81

B. E. Opitz K6-78

A. T. Owen K6-78

T. M. Poston K $6-13$

G. W. Patton K6-13

J. J. Reck K6-78

J. T. Rieger K6-96

D. R. Sauer $K \sigma-78$

M. L. Sours K6-01

F. A. Spane K6-96

D. L. Stewart K6-96

B. L. Tiller K6-13

T. L. VanArsdale K6-78

T. L. Wilson K6-78

V. C. Withers K6-78

R. K. Woodruff K6-13

Historical File--R. K. Woodruff

Publishing Coordination

Technical Report Files (5)

\section{Routing}

R. M. Ecker

M. J. Graham

P. M. Irving

R. L. Skaggs

C. S. Sloane

P. C. Hays (1 ast) 

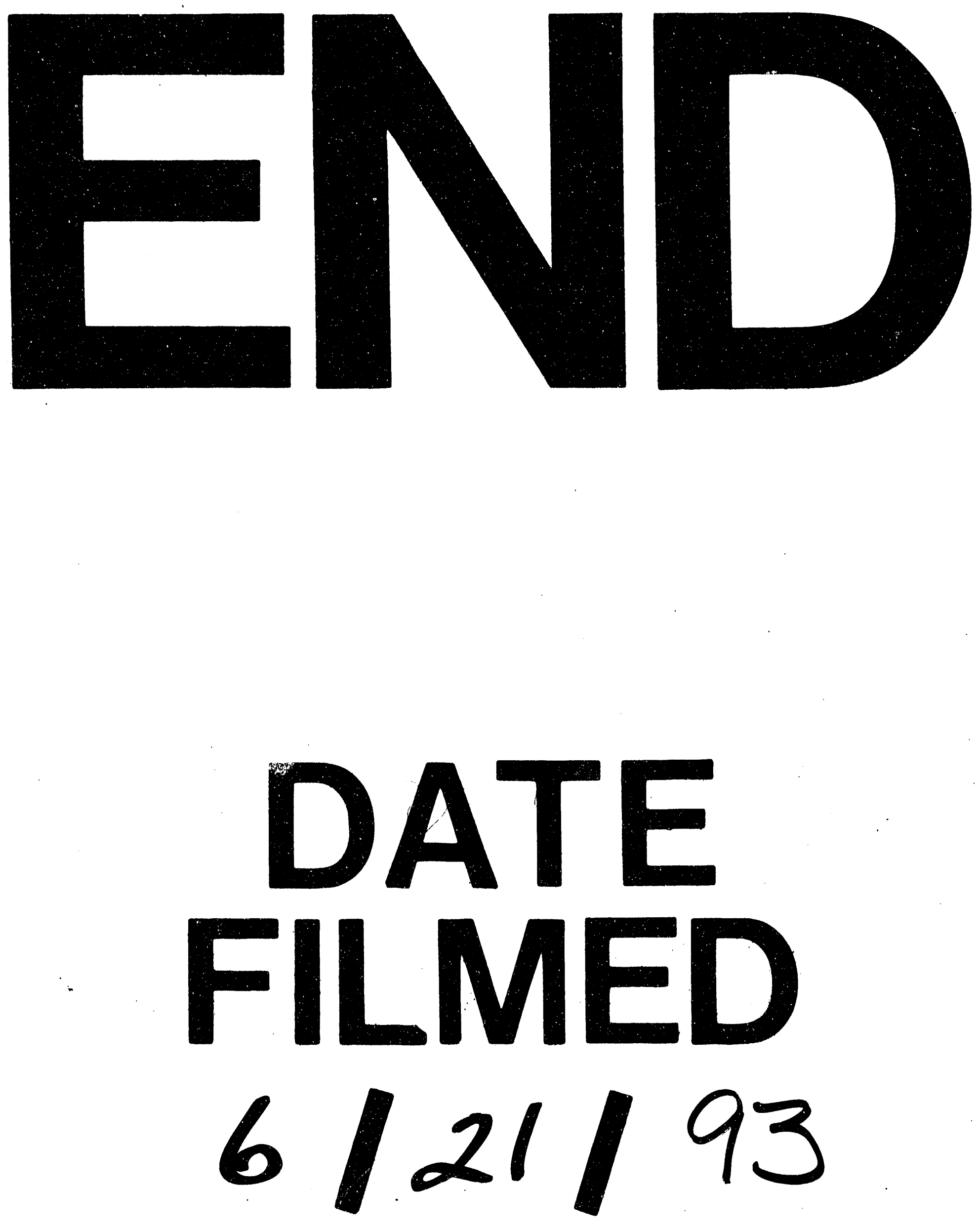

1 
